Pure and Applied Mathematics Quarterly

Volume 6, Number 4

(Special Issue: In honor of

Joseph J. Kohn, Part 2 of 2$)$

$1169-1241,2010$

\title{
Effective Termination of Kohn's Algorithm for Subelliptic Multipliers
}

\author{
Yum-Tong Siu* \\ Dedicated to Professor Joseph J. Kohn on his 75th Birthday
}

\begin{abstract}
This note discusses the problem of the effective termination of Kohn's algorithm for subelliptic multipliers for bounded smooth weakly pseudoconvex domains of finite type. We give a complete proof for the case of special domains of finite type and indicate briefly how this method is to be extended to the case of general bounded smooth weakly pseudoconvex domains of finite type.

Keywords: Subelliptic multipliers. Kohn's algorithm. Complex Neumann problem. Pseudoconvex domains. Subelliptic estimates. Finite Type. Multiplier ideal sheaves. Effective termination. Algebraic geometric techniques in analysis.
\end{abstract}

Introduction. In this note we will discuss the problem of the effective termination of Kohn's algorithm for subelliptic multipliers for bounded smooth weakly pseudoconvex domains of finite type [Ko79]. We will give a complete proof for the case of special domains and will only indicate briefly how this method is to be extended to the case of general bounded smooth weakly pseudoconvex domains of finite type. The method is rather simple and uses some local theory of algebraic geometry. People with some minimal background in algebraic geometry may find the algebraic-geometric techniques involved in this note very simple or

Received July 15, 2008.

*Partially supported by Grant DMS-0500964 from the National Science Foundation. 
even completely trivial. Since this topic is of interest mainly to the analysts I will use as much as possible the language of function theory to describe our method.

In a number of conferences in recent years I gave talks on this topic, but because of time limitation never had the opportunity to present all the details. This note is written to make the details available. This note will appear in the special issue of Pure and Applied Mathematics Quarterly for Professor Joseph J. Kohn.

The termination of Kohn's algorithm in the real-analytic case was verified by Diederich-Fornaess [DF78] without effectiveness. In this note we are also going to formulate Kohn's algorithm geometrically in terms of the theorem of Frobenius on integral submanifolds and present a proof from this geometric viewpoint so that one can see clearly how the procedures of Kohn's algorithm arise naturally in the geometric context and why the real-analyticity facilitates the proof of the termination of Kohn's algorithm. We present this more geometric proof here to provide an alternative to the proof of Proposition 3 on pp.380-388 of [DF78] which is the key step of [DF78] and which is still quite a bit of a challenge to follow. Moreover, the proof of the real-analytic case of the ineffective termination of Kohn's algorithm from the geometric viewpoint gives a better understanding of the rôle played by the real-analytic assumption and of what stands as a hurdle between generalizing the ineffective real-analytic case to the ineffective smooth case.

We also hope that this geometric viewpoint will provide an easier and more transparent setting for further developments of the application of algebraic-geometric techniques to general partial differential equations which Kohn has pioneered with his algorithm for the complex Neumann problem [Ko79]. The key point of Kohn's theory is the following. If the distribution of jets, where the partial-differentialequation estimate fails to hold, is not integrable even over unreduced points (or Artinian subschemes) of arbitrarily high order, then there is an algorithm to apply algebraic-geometric techniques to derive the partial-differential-equation estimate. Kohn implemented his theory for the complex Neumann problem. His theory should be applicable to systems of partial differential equations in a more general setting. Such an expected further development of his theory remains yet to be carried out. 
In a private communication Kohn told me that he has a direct proof of the result of Diederich-Fornaess [DF78] on the ineffective termination of the Kohn algorithm for the real-analytic case by using power series expansion of the realanalytic defining function and explicitly keeping track of various partial differentiations in the holomorphic and anti-holomorphic directions.

Andreea Nicoara recently posted an article [Ni07] in which she treats the ineffective termination of Kohn's algorithm for smooth weakly pseudoconvex domains of finite type from the viewpoint of adapting the ineffective argument of DiederichFornaess for the real-analytic case to the smooth case by using Tougeron elements [To72] and Catlin's multitype [Ca84] to examine the possibilities of removing the difficulties of the smooth case such as those arising from the existence of nonidentically-zero smooth function germs at a point whose derivatives of all orders vanish at that point.

At the end we include in this note an appendix which presents some techniques of applying Skoda's theorem on ideal generation [Sk72, Th.1, pp.555-556] which involve derivatives of functions and Jacobian determinants. Though most of these techniques are not directly used in this note (except the use of (A.2) in (III.7) and the use (A.3) in (III.8)), they may be useful in reducing vanishing orders of multiplier ideals in Kohn-type algorithms for more general partial differential equations.

An earlier version of this note was posted in arxiv.org server as arXiv:0706.4113. In its Proposition (III.6) on the Multiplicity Estimate from Adjunction Formula, when we differentiate a given multiplier inside the formation of a Jacobian determinant to construct another multiplier, we should have performed the differentiation as many times as the multiplicity of the given multiplier instead of performing it only once in Proposition (III.6) there. In this version we put in the correct number of differentiation. In (III.10) below we explain why the correct number of differentiation is necessary. In order to make our argument more transparent with minimum notational complexity, we first do the special case of complex dimension two. As a result we change completely the presentation of our argument in this version. The presentation here is more streamlined than in the earlier version. 
Before we go into the main body of this note, we would like to make one remark about the meaning of the effective termination of Kohn's algorithm. Kohn's algorithm tells us that multipliers can be produced by using Jacobian determinants or by taking roots. More precisely, taking a root means choosing an element in the radical of the ideal formed by multipliers in the preceding steps. The challenge in solving the problem of the effective termination of Kohn's algorithm is to come up with a procedure which specifies when a root should be taken and when a Jacobian determinant should be used. The procedure should specify when and how to choose an element in the radical of the ideal formed by multipliers in the preceding steps. It also should specify when and how to choose functions (either multipliers from the preceding steps or pre-multipliers in the sense of (III.6)) to form the Jacobian determinant. If one simply follows the rule of always giving priority to taking roots or simply follows the rule of always giving priority to taking Jacobian determinants, it is very easy to come up with simple counterexamples which show that such simple-minded rules could not in general yield an effective termination of Kohn's algorithm. Of course, the uselessness of such simple-minded rules does not mean that Kohn's algorithm cannot be effectively terminated. The problem of the effective termination of Kohn's algorithm is the determination of a selection rule to specify when and how to take an element in the radical of an ideal formed by multipliers from the previous steps and to specify when and how to take Jacobian determinants to form new multipliers. The purpose of this note is to present such a selection rule and thereby demonstrate the effective termination of Kohn's algorithm.

\section{Part I. Global Regularity, Subellipticity, Kohn's Algorithm, and Spe- cial Domains}

(I.1) The Setting. We start out with the setting of a bounded domain $\Omega$ in $\mathbb{C}^{n}$ with smooth boundary defined by $r<0$ for some smooth function $r$ on an open neighborhood of the topological closure of $\Omega$. We assume that $\Omega$ is weakly pseudoconvex at all its boundary points in the sense that at any boundary point $P$ of $\Omega$ the $(1,1)$-form $\partial \bar{\partial} r$ is weakly positive definite when restricted to the complex tangent space $\{\partial r=0\}$ of the boundary $\partial \Omega$ of $\Omega$ at $P$. (To be more precise, we should have said that $\sqrt{-1} \partial \bar{\partial} r$ is weakly positive definite instead of 
$\partial \bar{\partial} r$ being weakly positive definite, but for notational simplicity we will drop the factor $\sqrt{-1}$ if there is no risk of any confusion.)

The type $m$ at a point $P$ of the boundary $\partial \Omega$ of $\Omega$ is the supremum of the normalized touching order

$$
\frac{\operatorname{ord}_{0}(r \circ \varphi)}{\operatorname{ord}_{0} \varphi}
$$

to $\partial \Omega$, of all local holomorphic curves $\varphi: \Delta \rightarrow \mathbb{C}^{n}$ with $\varphi(0)=P$, where $\Delta$ is the open unit 1-disk and $\operatorname{ord}_{0}$ is the vanishing order at the origin 0 . A point $P$ of the boundary $\partial \Omega$ of $\Omega$ is said to be of finite type if the type $m$ at $P$ is finite. This notion of finite type was introduced by D'Angelo [DA79]. For notational convenience we also call $m$ the order of finite type instead of just the "type" to indicate that it is in the sense of D'Angelo's notion of finite type.

Our goal is to obtain global regularity for any smooth weakly pseudoconvex domain $\Omega$ of finite type in the sense that, for any $\bar{\partial}$-closed smooth $(0,1)$-form $f$ on $\Omega$ which is smooth up to the boundary of $\Omega$, the solution $u$ of $\bar{\partial} u=f$ on $\Omega$ with $u$ orthogonal to all holomorphic functions on $\Omega$ must also be smooth up to the boundary of $\Omega$. In this note by "smoothness" we mean infinite differentiability. For notational simplicity we formulate global regularity only for $(0,1)$-forms. It can be similarly formulated for $(0, q)$-forms for a general $q$.

Global regularity is a consequence of the subelliptic estimate, which is defined as follows. For any $P \in \partial \Omega$ there exist an open neighborhood $U$ of $P$ in $\mathbb{C}^{n}$ and positive numbers $\epsilon$ and $C$ satisfying

$$
\||g|\|_{\epsilon}^{2} \leq C\left(\|\bar{\partial} g\|^{2}+\left\|\bar{\partial}^{*} g\right\|^{2}+\|g\|^{2}\right)
$$

for any $(0,1)$-form $g$ supported on $U \cap \bar{\Omega}$ which is in the domain of $\bar{\partial}$ and $\bar{\partial}^{*}$, where $\||\cdot|\|_{\epsilon}$ is the $L^{2}$ norm on $\Omega$ involving derivatives up to order $\epsilon$ in the boundary tangential direction of $\Omega$ and $\|\cdot\|$ is the usual $L^{2}$ norm on $\Omega$ without involving any derivatives, and $\bar{\partial}^{*}$ is the actual adjoint of $\bar{\partial}$ with respect to $\|\cdot\|$.

Kohn [Ko79] introduced the following notion of multipliers to obtain the subelliptic estimate. At a point $P$ of $\partial \Omega$ a smooth function germ $f$ at $P$ is called a subelliptic multiplier (or simply called a multiplier) if there exist some open neighborhood $U$ of $P$ in $\mathbb{C}^{n}$ and some positive numbers $\epsilon$ and $C$ (all three depending on $f$ ) such that

$$
\||f g|\|_{\epsilon}^{2} \leq C\left(\|\bar{\partial} g\|^{2}+\left\|\bar{\partial}^{*} g\right\|^{2}+\|g\|^{2}\right)
$$


for any $(0,1)$-form $g$ supported on $U \cap \bar{\Omega}$ which is in the domain of $\bar{\partial}$ and $\bar{\partial}^{*}$. We call the positive number $\varepsilon$ an order of subellipticity for the multiplier $f$. We also call a subelliptic multiplier a scalar multiplier to emphasize its difference from vector-multipliers introduced below. The collection of all multipliers at $P$ forms a ideal which is called the multiplier ideal and is denoted by $I_{P}$.

A germ of a smooth $(1,0)$-form $\theta$ at $P$ is called a subelliptic vector-multiplier if there exist some open neighborhood $U$ of $P$ in $\mathbb{C}^{n}$ and some positive numbers $\epsilon$ and $C$ (all three depending on $\theta$ ) such that

$$
\|\bar{\theta} \cdot g \mid\|_{\epsilon}^{2} \leq C\left(\|\bar{\partial} g\|^{2}+\left\|\bar{\partial}^{*} g\right\|^{2}+\|g\|^{2}\right)
$$

for any $(0,1)$-form $g$ supported on $U \cap \bar{\Omega}$ which is in the domain of $\bar{\partial}$ and $\bar{\partial}^{*}$, where $\bar{\theta} \cdot g$ is the function obtained by taking the pointwise inner product of the complex-conjugate $\bar{\theta}$ of $\theta$ with $g$ with respect to the Euclidean metric of $\mathbb{C}^{n}$. We call the positive number $\varepsilon$ an order of subellipticity for the vector-multiplier $\theta$. The collection of all vector-multipliers at $P$ forms a module which is called the vector-multiplier module and is denoted by $A_{P}$.

The subelliptic estimate holds at a boundary point $P$ of $\Omega$ if a nonzero constant function belongs to $I_{P}$. Kohn introduced the following algorithm to generate elements of $I_{P}$.

(A) Initial Membership.

(i) $r \in I_{P}$.

(ii) $\partial \bar{\partial}_{j} r$ belongs to $A_{P}$ for every $1 \leq j \leq n-1$ if $\partial r=d z_{n}$ at $P$ for some local holomorphic coordinate system $\left(z_{1}, \cdots, z_{n}\right)$ centered at $P$, where $\partial_{j}$ means $\frac{\partial}{\partial z_{j}}$.

(B) Generation of New Members.

(i) If $f \in I_{P}$, then $\partial f \in A_{P}$.

(ii) If $\theta_{1}, \cdots, \theta_{n-1} \in A_{P}$, then the coefficient of

$$
\theta_{1} \wedge \cdots \wedge \theta_{n-1} \wedge \partial r
$$

is in $I_{P}$.

(C) Real Radical Property.

If $g \in I_{P}$ and $|f|^{m} \leq|g|$ for some positive integer $m$, then $f \in I_{P}$.

The key point of Kohn's algorithm is to allow certain differential operators to lower the vanishing orders of multipliers so that eventually one can get a nonzero 
constant as a multiplier. However, there are two limitations on this process of differentiation to lower vanishing orders. One is that only $(1,0)$-differentiation is allowed (as formulated in (B)(i) above). The other is that only determinants of coefficients of (1,0)-differentials can be used (as formulated in (B)(ii) above). Besides using differentiation the "real radical property" allows root-taking to reduce vanishing orders (as formulated in (C) above).

For the proof of the effective termination of the Kohn algorithm, to keep track of the effectiveness we will assign a positive number to a scalar multiplier (respectively a vector-multiplier) which is an order of subellipticity for the scalar multiplier (respectively vector multiplier). We call such a positive number an assigned order of subellipticity. In addition to keeping track of the number and the nature of the steps of the Kohn algorithm used, the effectiveness of the termination of the Kohn algorithm seeks to keep track of the assigned orders of subellipticity for the individual scalar multipliers and vector-multipliers so that the final nowhere zero multiplier has an effective positive number as its assigned order of subellipticity. Note that the assigned order of subellipticity of a scalar multiplier or vector-multiplier constructed in the Kohn algorithm is in general not the maximum $\varepsilon$ for the inequality (I.1.1) or (I.1.2).

We will adopt the following rule of giving to a scalar multiplier or a vectormultiplier in the Kohn algorithm its assigned order of multiplicity. As its assigned order of subellipticity we give the scalar multiplier $r$ the number 1 . As its assigned order of subellipticity we give the number $\frac{1}{2}$ to the vector-multiplier $\partial \bar{\partial}_{j} r$ at $P$ for every $1 \leq j \leq n-1$ is $\frac{1}{2}$ if $\partial r=d z_{n}$ at $P$ for some local holomorphic coordinate system $\left(z_{1}, \cdots, z_{n}\right)$ centered at $P$. If the assigned order of subellipticity of the scalar multiplier $f$ is $\varepsilon$, then we give to the vector-multiplier $\partial f$ as its assigned order of subellipticity the number $\frac{\varepsilon}{2}$. If the minimum of the assigned orders of subellipticity of the vector-multipliers $\theta_{1}, \cdots, \theta_{n-1}$ is $\varepsilon$, then we give to the same $\varepsilon$ to the coefficient of

$$
\theta_{1} \wedge \cdots \wedge \theta_{n-1} \wedge \partial r
$$

as a scalar multiplier as its assigned order of subellipticity. If the assigned order of subellipticity of the scalar multiplier $g$ is $\varepsilon$ and if $|f|^{m} \leq|g|$ for some positive integer $m$, then we give the number $\frac{\varepsilon}{m}$ to the scalar multiplier $f$ as its assigned order of subellipticity. 
(I.2) Algebraic-Geometric Description of Finite Type for Special Domains. A special domain $\Omega$ in $\mathbb{C}^{n+1}$ (with coordinates $w, z_{1}, \cdots, z_{n}$ ) is a bounded domain given by

$$
\operatorname{Re} w+\sum_{j=1}^{N}\left|F_{j}\left(z_{1}, \cdots, z_{n}\right)\right|^{2}<0,
$$

where $F_{j}\left(z_{1}, \cdots, z_{n}\right)$ defined on some open neighborhood of $\bar{\Omega}$ in $\mathbb{C}^{n+1}$ depends only on the variables $z_{1}, \cdots, z_{n}$ and is holomorphic in $z_{1}, \cdots, z_{n}$ for each $1 \leq j \leq$ $N$. In what follows, when we consider the subelliptic estimate at a boundary point $P$ of $\Omega$ and its type of finite order, if there is no confusion we will assume without loss of generality and without explicit mention that the point $P$ is the origin of the coordinates $w, z_{1}, \cdots, z_{n}$ and that $F_{j}$ vanishes at $P$ for each $1 \leq j \leq N$. Such special domains were introduced by Kohn [Ko79, p.115, §7].

To quantitatively describe finite type at the origin in a way which is more convenient to use, we introduce a positive number $p$ which is the smallest positive integer such that

$$
|z|^{p} \leq A_{1} \sum_{j=1}^{N}\left|F_{j}(z)\right|
$$

on some open neighborhood of the origin in $\mathbb{C}^{n}$ for some positive constant $A_{1}$, where $z=\left(z_{1}, \cdots, z_{n}\right)$ and $|z|^{2}=\sum_{\ell=1}^{n}\left|z_{\ell}\right|^{2}$. We will verify below in (I.3) that the order of finite type at the origin $P$ is equal to $2 p$.

We are going to introduce also two other effectively comparable ways to describe finite type which are both algebraic-geometrical. The first one is the following. Let $\mathfrak{m}$ be the maximum ideal $\mathfrak{m}_{\mathbb{C}^{n}, 0}$ of $\mathbb{C}^{n}$ at the origin. Let $\mathcal{I}$ be the ideal on $\mathbb{C}^{n}$ generated by holomorphic function germs $F_{1}, \cdots, F_{N}$ on $\mathbb{C}^{n}$ at the origin. Let $q$ be the smallest positive integer such that

$$
\mathfrak{m}^{q} \subset \mathcal{I} \text {. }
$$

We will verify below in (I.4) that the number $p$ is related to the number $q$ by the inequality $p \leq q \leq(n+2) p$. This inequality is far from being sharp.

The second is the following. Let $s$ be the dimension over $\mathbb{C}$ of the quotient of $\mathcal{O}_{\mathbb{C}^{n}, 0}$ by the ideal generated in it by the holomorphic function germs $F_{1}, \cdots, F_{N}$ on $\mathbb{C}^{n}$ at the origin. We will verify below in (I.5) that the number $q$ is related to 
the number $s$ by the two inequalities $q \leq s$ and $s \leq\left(\begin{array}{c}n+q-1 \\ q-1\end{array}\right)$. Again this pair of inequalities is far from being sharp.

When we prove the effective termination of Kohn's algorithm for special domains, we will in different contexts choose to use one of the three effectively comparable descriptions $p, q$, or $s$ of the order of finite type.

Let $U$ be an open neighborhood of 0 in $\mathbb{C}$ and $\psi: U \rightarrow \mathbb{C}^{n+1}$ be a holomorphic map with $\psi(0)=0$. Write $\psi=\left(\psi_{0}, \psi_{1}\right)$ such that $\psi_{0}: U \rightarrow \mathbb{C}$ and $\psi_{1}: U \rightarrow \mathbb{C}^{n}$. For $j=0,1$ the vanishing order $\operatorname{ord}_{0} \psi_{j}$ at 0 of $\psi_{j}$ is the positive integer $s$ such that

$$
\psi_{j}(0)=\left(d \psi_{j}\right)(0)=\cdots=\left(d^{s-1} \psi_{j}\right)(0)=0
$$

and $\left(d^{s} \psi_{j}\right)(0) \neq 0$. This positive integer $s$ can also be described as the largest integer such that

$$
\left|\psi_{j}(\zeta)\right| \leq A_{2, j}|\zeta|^{s}
$$

for some positive constant $A_{2, j}$ when the coordinate $\zeta$ of $\mathbb{C}$ is small. The vanishing order $\operatorname{ord}_{0}(\psi)$ at the origin of $\psi$ is equal to the minimum of the vanishing orders $\operatorname{ord}_{0}\left(\psi_{0}^{*} w\right)$ and $\operatorname{ord}_{0}\left(\psi_{1}^{*} z_{j}\right)$ of the holomorphic functions $\psi_{0}^{*} w$ and $\psi_{1}^{*} z_{j}$ of $\zeta$ at $\zeta=0$ for $1 \leq j \leq n$.

In the expansion of the function

$$
\psi^{*} r=\operatorname{Re}\left(\left(\psi_{0}^{*} w\right)(\zeta)\right)+\sum_{j=1}^{N}\left|F_{j}\left(\psi_{1}(\zeta)\right)\right|^{2}
$$

in $\zeta, \bar{\zeta}$, only terms of the form $a_{\nu} \zeta^{\nu}$ and $b_{\nu} \bar{\zeta}^{\nu}$ with $\nu \geq 1$ (i.e., purely holomorphic or purely antiholomorphic terms in $\zeta$ ) can occur in $\operatorname{Re}\left(\left(\psi_{0}^{*} w\right)(\zeta)\right)$ and only terms of the form $c_{\mu, \nu} \zeta^{\mu} \bar{\zeta}^{\nu}$ with $\mu \geq 1$ and $\nu \geq 1$ (i.e., never purely holomorphic or purely antiholomorphic terms in $\zeta$ ) occur in $\sum_{j=1}^{N}\left|F_{j}\left(\psi_{1}(\zeta)\right)\right|^{2}$ (where $a_{\nu}, b_{\nu}, c_{\mu, \nu}$ are complex constants). Since there is no possibility at all of any term from $\operatorname{Re}\left(\left(\psi_{0}^{*} w\right)(\zeta)\right)$ canceling a term from $\sum_{j=1}^{N}\left|F_{j}\left(\psi_{1}(\zeta)\right)\right|^{2}$, it follows that the vanishing order at 0 of $\psi^{*} r$ must be equal always to the minimum of the order at 0 of $\operatorname{Re}\left(\left(\psi_{0}^{*} w\right)(\zeta)\right)$ and the order at 0 of $\sum_{j=1}^{N}\left|F_{j}\left(\psi_{1}(\zeta)\right)\right|^{2}$. Thus

$$
\frac{\operatorname{ord}_{0} \psi^{*} r}{\operatorname{ord}_{0} \psi}=\frac{\min \left(\operatorname{ord}_{0} \psi_{0}^{*} w, \operatorname{ord}_{0} \psi_{1}^{*} \sum_{j=1}^{N}\left|F_{j}\right|^{2}\right)}{\min \left(\operatorname{ord}_{0} \psi_{0}^{*} w, \frac{1}{2} \operatorname{ord}_{0} \psi_{1}^{*} \sum_{j=1}^{n}\left|z_{j}\right|^{2}\right)} .
$$


(I.3) Lemma. Let $p$ be the smallest positive integer which satisfies (I.2.2) for some positive constant $A_{1}$. Then the order $t$ of finite type at the origin for the special domain $\Omega$ given by (I.2.1) is equal to $2 p$.

Proof. We are going to prove $t=2 p$ by proving the two inequalities $t \leq 2 p$ and $t \geq 2 p$. We first prove the inequality $t \leq 2 p$. From the definition of the order $t$ of finite type we know that there exist some open neighborhood $U$ of 0 in $\mathbb{C}$ and some holomorphic map $\psi=\left(\psi_{0}, \psi_{1}\right): U \rightarrow \mathbb{C}^{n+1}=\mathbb{C} \times \mathbb{C}^{n}$ with $\psi(0)=0$ such that

We let

$$
t=\frac{\operatorname{ord}_{0} \psi^{*} r}{\operatorname{ord}_{0} \psi}=\frac{\min \left(\operatorname{ord}_{0} \psi_{0}^{*} w, \operatorname{ord}_{0} \psi_{1}^{*} \sum_{j=1}^{N}\left|F_{j}\right|^{2}\right)}{\min \left(\operatorname{ord}_{0} \psi_{0}^{*} w, \frac{1}{2} \operatorname{ord}_{0} \psi_{1}^{*} \sum_{j=1}^{n}\left|z_{j}\right|^{2}\right)} .
$$

$$
\alpha=\operatorname{ord}_{0} \psi_{0}^{*} w, \quad \beta=\frac{1}{2} \operatorname{ord}_{0} \psi_{1}^{*} \sum_{j=1}^{n}\left|z_{j}\right|^{2}, \quad \gamma=\operatorname{ord}_{0} \psi_{1}^{*} \sum_{j=1}^{N}\left|F_{j}\right|^{2} .
$$

From the definition of $p$ we have $\gamma \leq 2 p \beta$. We differentiate among the following three cases.

Case 1. $\alpha \leq \beta$.

Case 2. $\beta<\alpha \leq 2 p \beta$

Case 3. $\alpha>2 p \beta$.

For Case 1, we have

$$
t=\frac{\min (\alpha, \gamma)}{\min (\alpha, \beta)} \leq \frac{\min (\alpha, 2 p \beta)}{\min (\alpha, \beta)}=\frac{\alpha}{\alpha} \leq 2 p .
$$

For Case 2, we have

$$
t=\frac{\min (\alpha, \gamma)}{\min (\alpha, \beta)} \leq \frac{\min (\alpha, 2 p \beta)}{\min (\alpha, \beta)}=\frac{\alpha}{\beta} \leq \frac{2 p \beta}{\beta}=2 p .
$$

For Case 3, we have

$$
t=\frac{\min (\alpha, \gamma)}{\min (\alpha, \beta)} \leq \frac{\min (\alpha, 2 p \beta)}{\min (\alpha, \beta)}=\frac{2 p \beta}{\beta}=2 p .
$$

Thus in all three cases $t \leq 2 p$.

We are now going to prove the other inequality $2 p \leq t$. We use a simultaneous resolution of embedded singularities $\pi: \tilde{W} \rightarrow W$ for some open neighborhood $W$ of the origin in $\mathbb{C}^{n}$ with exceptional hypersurfaces $\left\{Y_{j}\right\}_{j=1}^{J}$ in $\tilde{W}$ in normal crossing so that the pullback $\pi^{*} \mathfrak{m}$ of the maximum ideal on $W$ at the origin is equal to the 
ideal sheaf of the divisor $\sum_{j=1}^{J} \sigma_{j} Y_{j}$ for some nonnegative integers $\sigma_{1}, \cdots, \sigma_{J}$ and the pullback $\pi^{*} \mathcal{I}$ of the ideal sheaf $\mathcal{I}$ on $\mathbb{C}^{n}$ generated by $F_{1}, \cdots, F_{N}$ is equal to the ideal sheaf of the divisor $\sum_{j=1}^{J} \tau_{j} Y_{j}$ for some nonnegative integers $\tau_{1}, \cdots, \tau_{J}$.

Since $p$ is the smallest integer which satisfies condition (I.2.2) for some positive constant $A_{1}$, it follows that $p \sigma_{j} \leq \tau_{j}$ for all $1 \leq j \leq J$. Take any $1 \leq j_{0} \leq J$ with $\sigma_{j_{0}}>0$ and $0 \in \pi\left(Y_{j_{0}}\right)$ such that there is a regular point $Q$ of some $Y_{j_{0}}$ with the property that $\pi(Q)=0$ and $Q$ does not belong to any other $Y_{j}$ with $j \neq j_{0}$. Take a local regular complex curve $\tilde{C}$ in $\tilde{W}$ represented by a holomorphic map $\tilde{\varphi}: U \rightarrow \tilde{W}$ from some open neighborhood $U$ of 0 in $\mathbb{C}$ to $\tilde{W}$ such that $\tilde{\varphi}(0)=Q$ and the local complex curve $\tilde{C}$ is transversal to $Y_{j_{0}}$ and disjoint from any other $Y_{j}$ with $j \neq j_{0}$. Now we define a holomorphic map $\psi=\left(\psi_{0}, \psi_{1}\right): U \rightarrow \mathbb{C}^{n+1}=\mathbb{C} \times \mathbb{C}^{n}$ by $\psi_{0} \equiv 0$ and $\psi_{1}=\pi \circ \varphi$. Then

$$
\begin{gathered}
\frac{\operatorname{ord}_{0} \psi^{*} r}{\operatorname{ord}_{0} \psi}=\frac{\min \left(\operatorname{ord}_{0} \psi_{0}^{*} w, \operatorname{ord}_{0} \psi_{1}^{*} \sum_{j=1}^{N}\left|F_{j}\right|^{2}\right)}{\min \left(\operatorname{ord}_{0} \psi_{0}^{*} w, \frac{1}{2} \operatorname{ord}_{0} \psi_{1}^{*} \sum_{j=1}^{n}\left|z_{j}\right|^{2}\right)} \\
=\frac{\operatorname{ord}_{0} \psi_{1}^{*} \sum_{j=1}^{N}\left|F_{j}\right|^{2}}{\frac{1}{2} \operatorname{ord}_{0} \psi_{1}^{*} \sum_{j=1}^{n}\left|z_{j}\right|^{2}}=\frac{2 \tau_{j_{0}}}{\sigma_{j_{0}}} \geq 2 p .
\end{gathered}
$$

By the definition of $t$ we have $t \geq 2 p$. Putting the two inequalities $t \leq 2 p$ and $t \geq 2 p$ together, we get $t=2 p$. Q.E.D.

(I.4) Lemma. Let $p$ be the smallest positive integer which satisfies (I.2.2) for some positive constant $A_{1}$. Let $q$ be the smallest positive integer such that the $q$-th power $\mathfrak{m}^{q}$ of the maximum ideal $\mathfrak{m}$ of $\mathbb{C}^{n}$ at the origin is contained in the ideal $\mathcal{I}$ generated by the holomorphic function germs $F_{1}, \cdots, F_{N}$ on $\mathbb{C}^{n}$ at the origin. Then $p \leq q \leq(n+2) p$.

Proof. From the definition of $q$ it follows that

$$
z_{\ell}^{q} \in \mathfrak{m}^{q} \subset \mathcal{I}=\sum_{j=1}^{N} \mathcal{O}_{\mathbb{C}^{n}, 0} F_{j}
$$

for every $1 \leq \ell \leq n$ it follows that

$$
\left|z_{\ell}^{q}\right| \leq \tilde{A}_{\ell} \sum_{j=1}^{N}\left|F_{j}(z)\right|
$$


for some positive constant $\tilde{A}_{\ell}$ for $1 \leq \ell \leq n$ on some open neighborhood of the origin in $\mathbb{C}^{n}$. Hence

$$
|z|^{q}=\left(\sum_{\ell=1}^{n}\left|z_{\ell}\right|^{2}\right)^{\frac{q}{2}} \leq n^{\frac{q}{2}} \max _{1 \leq \ell \leq n}\left|z_{\ell}\right|^{q} \leq n^{\frac{q}{2}}\left(\max _{1 \leq \ell \leq n} \tilde{A}_{\ell}\right) \sum_{j=1}^{N}\left|F_{j}(z)\right|
$$

and $p \leq q$ from the definition of $p$.

For the proof of the inequality $p \leq(n+2) q$, we need the following theorem of Skoda [Sk72, Th.1, pp.555-556].

Let $D$ be a pseudoconvex domain in $\mathbf{C}^{n}$ and $\chi$ be a plurisubharmonic function on $D$. Let $g_{1}, \ldots, g_{m}$ be holomorphic functions on $D$. Let $\alpha>1$ and $\ell=$ $\inf (n, m-1)$. Then for every holomorphic function $F$ on $D$ such that

$$
\int_{D}|F|^{2}|g|^{-2 \alpha \ell-2} e^{-\chi}<\infty
$$

there exist holomorphic functions $f_{1}, \ldots, f_{m}$ on $\Omega$ such that

$$
F=\sum_{j=1}^{m} g_{j} f_{j}
$$

and

where

$$
\int_{D}|f|^{2}|g|^{-2 \alpha \ell} e^{-\chi} \leq \frac{\alpha}{\alpha-1} \int_{D}|F|^{2}|g|^{-2 \alpha \ell-2} e^{-\chi}
$$

$$
|g|=\left(\sum_{j=1}^{m}\left|g_{j}\right|^{2}\right)^{\frac{1}{2}}, \quad|f|=\left(\sum_{j=1}^{m}\left|f_{j}\right|^{2}\right)^{\frac{1}{2}} .
$$

For nonnegative integers $\gamma_{1}, \cdots, \gamma_{n}$ with $\gamma_{1}+\cdots+\gamma_{n}=(n+2) p$ we apply Skoda's theorem to the case of

$$
\begin{gathered}
F=\prod_{j=1}^{n} z_{j}^{\gamma_{j}}, \quad m=N+n, \\
\chi \equiv 0, \quad\left\{g_{1}, \cdots, g_{m}\right\}=\left\{F_{1}, \cdots, F_{N}, 0, \cdots, 0\right\}, \\
\ell=n, \quad \alpha=\frac{n+1}{n},
\end{gathered}
$$

with $D$ being some small open ball neighborhood of the origin in $\mathbb{C}^{n}$, to conclude from (I.2.2) that

$$
\prod_{j=1}^{n} z_{j}^{\gamma_{j}} \in \mathcal{I}=\sum_{j=1}^{N} \mathcal{O}_{\mathbb{C}^{n}, 0} F_{j}
$$


Hence $q \leq(n+2) p$. Q.E.D.

(I.5) Lemma. Let $q$ be the smallest positive integer such that the $q$-th power $\mathfrak{m}^{q}$ of the maximum ideal $\mathfrak{m}$ of $\mathbb{C}^{n}$ at the origin is contained in the ideal $\mathcal{I}$ generated by the holomorphic function germs $F_{1}, \cdots, F_{N}$ on $\mathbb{C}^{n}$ at the origin. Let $s$ be the dimension over $\mathbb{C}$ of the quotient of $\mathcal{O}_{\mathbb{C}^{n}, 0}$ by the ideal $\mathcal{I}$. Then $q \leq s$ and $s \leq\left(\begin{array}{c}n+q-1 \\ q-1\end{array}\right)$.

Proof. Let $R=\mathcal{O}_{\mathbb{C}^{n}, 0}$. Since $\mathfrak{m}^{q} \subset \mathcal{I}$, it follows that

$$
s=\operatorname{dim}_{\mathbb{C}} R / \mathcal{I} \leq \operatorname{dim}_{\mathbb{C}} R / \mathfrak{m}^{q}=\left(\begin{array}{c}
n+q-1 \\
q-1
\end{array}\right) .
$$

On the other hand, we consider the following sequence of nested $\mathbb{C}$-linear subspaces of the finite-dimensional $\mathbb{C}$-vector spaces $R / \mathcal{I}$ of complex dimension $s$.

$$
R / \mathcal{I} \supset \mathfrak{m}(R / \mathcal{I}) \supset \mathfrak{m}^{2}(R / \mathcal{I}) \supset \cdots \supset \mathfrak{m}^{\ell}(R / \mathcal{I}) \supset \mathfrak{m}^{\ell+1}(R / \mathcal{I}) \supset \cdots
$$

There exists some $1 \leq \ell \leq s$ such that

$$
\mathfrak{m}^{\ell}(R / \mathcal{I})=\mathfrak{m}^{\ell+1}(R / \mathcal{I}) .
$$

By Nakayama's lemma we have

$$
0=\mathfrak{m}^{\ell}(R / \mathcal{I})=\left(\mathfrak{m}^{\ell}+\mathcal{I}\right) / \mathcal{I}
$$

and we conclude that $\mathfrak{m}^{\ell}+\mathcal{I}=\mathcal{I}$ and $\mathfrak{m}^{\ell} \subset \mathcal{I}$, which implies that $q \leq s$. Q.E.D.

The following corollary of Lemma (I.5) is a version of the effective Nullstellensatz in terms of multiplicity.

(I.6) Lemma. Let $\mathcal{I}$ be an ideal in $\mathcal{O}_{\mathbb{C}^{n}, 0}$ such that its multiplicity is no more than some positive integer $m$ in the sense that the complex dimension of the quotient of $\mathcal{O}_{\mathbb{C}^{n}, 0}$ by $\mathcal{I}$ is no more than $m$. Let $f$ be a holomorphic function germ on $\mathbb{C}^{n}$ at the origin which vanishes at the origin. Then $f^{m^{2}}$ belongs to $\mathcal{I}$.

Proof. Let $\mathcal{I}=\bigcap_{j=1}^{J} \mathcal{Q}_{j}$ be the primary decomposition of the ideal $\mathcal{I}$ in the Noetherian ring $\mathcal{O}_{\mathbb{C}^{n}, 0}$ and let $\mathcal{P}_{j}$ be the radical of the primary ideal $\mathcal{Q}_{j}$. Since $m$ is the multiplicity of $\mathcal{I}$, we have $J \leq m$. Let $Z_{j}$ be the subvariety germ of $\mathbb{C}^{n}$ at the origin whose ideal at the origin is $\mathcal{P}_{j}$ and let $Z_{j}^{0}$ be the set of all regular points of $Z_{j}$, which without loss of generality we can assume to be connected. Let $n_{j}$ be the complex codimension of $Z_{j}$ at the origin. Take a generic point $Q_{j}$ in $Z_{j}^{0}$ and let $\Pi_{j}$ be a complex $\mathbb{C}$-linear subspace of complex dimension $n_{j} \mathbb{C}^{n}$ 
which is transversal to $Z_{j}^{0}$ at the point $Q_{j}$. The multiplicity of the ideal at $Q_{j}$ induced by $\mathcal{Q}_{j}$ is no more than $m$ (if we assume without loss of generality that $Q_{j}$ is sufficiently close to the origin). Let $\mathcal{J}_{j}$ be the ideal on $\Pi_{j} \approx \mathbb{C}^{n_{j}}$ at $Q_{j}$ induced by $\mathcal{Q}_{j}$. Then the multiplicity of $\mathcal{J}_{j}$ at the point $Q_{j}$ is no more than $m$. By Lemma (I.5) applied to $\Pi_{j} \approx \mathbb{C}^{n_{j}}$ and the ideal $\mathcal{J}_{j}$ on $\Pi_{j} \approx \mathbb{C}^{n_{j}}$, we conclude that the holomorphic function germ $\left(\left.f\right|_{\Pi_{j}}\right)^{m}$ on $\Pi_{j} \approx \mathbb{C}^{n_{j}}$ at $Q_{j}$ belongs to the ideal $\mathcal{J}_{j}$. Since $Q_{j}$ is a generic point in $Z_{j}^{0}$ and since $\mathcal{Q}_{j}$ is a primary ideal, it follows that the holomorphic function germ $f^{m}$ on $\mathbb{C}^{n}$ at the origin belongs to $\mathcal{Q}_{j}$. From $J \leq m$ it follows that the holomorphic function germ $f^{m^{2}}$ on $\mathbb{C}^{n}$ at the origin belongs to the product of $\mathcal{Q}_{j}$ for $1 \leq j \leq J$. In particular, holomorphic function germ $f^{m^{2}}$ on $\mathbb{C}^{n}$ at the origin belongs to $\mathcal{I}$. Q.E.D.

\section{Part II. Algebraic Formulation and Sketches of Techniques}

(II.1) Algebraic Formulation of Kohn's Algorithm for Special Domains. The effective termination of Kohn's algorithm for a special domain is reduced to the purely algebraic-geometric description of items (i) through (vii) listed below. For the case of a special domain the setting is as follows. We have a special domain $\Omega$ in $\mathbb{C}^{n+1}$ (with coordinates $w, z_{1}, \cdots, z_{n}$ ) defined by

$$
r:=\operatorname{Re} w+\sum_{j=1}^{N}\left|F_{j}\left(z_{1}, \cdots, z_{n}\right)\right|^{2}<0,
$$

where for each $1 \leq j \leq N, F_{j}\left(z_{1}, \cdots, z_{n}\right)$ is a holomorphic function vanishing at the origin which is independent of $w$ and is defined on some open neighborhood of $\bar{\Omega}$ in $\mathbb{C}^{n+1}$. The boundary point of $\Omega$ under consideration is the origin of $\mathbb{C}^{n+1}$.

In this setting, first of all, from $d r=d w$ at the origin and

$$
\partial \bar{\partial} r=\sum_{j=1}^{N} d F_{j} \wedge \overline{d F_{j}}
$$

we conclude from (I.1)(A)(ii) and standard techniques of estimates in Kohn's theory of multipliers [Ko79] that $d F_{j}$ is a vector multiplier which can be given $\frac{1}{4}$ as its assigned order of subellipticity, because the vector-multiplier

$$
\partial\left(\frac{\partial r}{\partial \overline{z_{j}}}\right)=\sum_{\ell=1}^{N} \overline{\left(\frac{\partial h_{\ell}}{\partial z_{j}}\right)} d h_{\ell}
$$


at the origin can be given $\frac{1}{2}$ as its assigned order of subellipticity for $1 \leq j \leq n$.

(i) We start out with the $N$ given holomorphic function germs $F_{1}, \cdots, F_{N}$ on $\mathbb{C}^{n}$ at the origin with the origin as their only common zero-point. The multiplicity $q$ of the ideal generated by $F_{1}, \cdots, F_{N}$ at the origin is what we use for effectiveness statements. That is, a number is considered effective if it can be estimated by an explicit expression in $q$.

(ii) Select $n \mathbb{C}$-linear combinations $g_{1}, \cdots, g_{n}$ of $F_{1}, \cdots, F_{N}$.

(iii) Form the Jacobian determinant of $g_{1}, \cdots, g_{n}$ with respect to $z_{1}, \cdots, z_{n}$.

(iv) Take the ideal $I$ generated by all such Jacobian determinants.

(v) Choose a finite subset $\varphi_{1}, \cdots, \varphi_{\ell}$ of the radical $J$ of $I$ and let $\sigma$ be a positive number such that $\left(\varphi_{j}\right)^{\sigma} \in I$ for $1 \leq j \leq \ell$.

(vi) Replace the set $F_{1}, \cdots, F_{N}$ by $F_{1}, \cdots, F_{N}, \varphi_{1}, \cdots, \varphi_{\ell}$ and repeat the above procedure.

(vi) Repeat until we get to the point that $\varphi_{1}$ can be chosen to be nonzero at the origin.

(vii) Effectiveness means that we have an effective number of steps and also an effective bound on $\sigma$ at each step.

(II.2) Sketch of Proof of Effectiveness for Special Domains. We now give an overview of the logical framework for the proof of the effective termination of the Kohn algorithm for special domains. Details for the derivation of the bounds of the multiplicities of functions constructed from generic $\mathbb{C}$-linear combinations and Jacobian determinants which occur in this overview will not be explained here but will be presented later in (III.3), (III.4), and (III.5).

We start out with an ideal generated by holomorphic function germs $F_{1}, \cdots, F_{N}$ on $\mathbb{C}^{n}$ at the origin whose common zero-set is the origin. The multiplicity $q$ of the ideal generated by $F_{1}, \cdots, F_{N}$ at the origin is what we use for effectiveness statements. For $n$ generic $\mathbb{C}$-linear combinations $g_{1}, \cdots, g_{n}$ of $F_{1}, \cdots, F_{N}$ the multiplicity of the function $f$ defined by $d g_{1} \wedge \cdots \wedge d g_{n}=f\left(d z_{1} \wedge \cdots \wedge d z_{n}\right)$ is no more than $m_{q}$ at the origin, where $m_{q}$ is some positive integer depending effectively on $q$ (see (III.5)). The main idea is to use the procedure of replacing $\mathbb{C}^{n}$ 
by the subspace $V$ defined by the multiplier $f$ to cut down successively on the dimension of the zero-set of multipliers while maintaining effectiveness.

There are two difficulties here. One difficulty is that the subspace defined by $f$ is in general not regular. The other difficulty is that we are allowed only to form Jacobian determinants of $\mathbb{C}$-linear combinations $g_{1}, \cdots, g_{n}$ of $F_{1}, \cdots, F_{N}$ and not allowed to form the Jacobian determinants of the restrictions of such $\mathbb{C}$-linear combinations $g_{1}, \cdots, g_{n-1}$ to $V$. The two difficulties are related. If $V$ is nonsingular, we could compute the Jacobian determinant of $\left.g_{1}\right|_{V}, \cdots,\left.g_{n-1}\right|_{V}$ by computing the coefficient of $d z_{1} \wedge \cdots \wedge d z_{n}$ in $d g_{1} \wedge \cdots \wedge d g_{n-1} \wedge d f$.

When $V$ is singular at the origin, we have to differentiate $f$ not just once to form $d f$ but as many times as the multiplicity of $V$. To enable us to do it by using Jacobian determinants, we construct a Weierstrass polynomial $\tilde{f}$ in $z_{n}$ whose coefficients are functions of $g_{1}, \cdots, g_{n-1}$ so that $\tilde{f}$ vanishes on the subspace $V$ and therefore contains $f$ as a factor. We then differentiate $\tilde{f}$ as many times as its multiplicity at the origin by applying the operator $d g_{1} \wedge \cdots \wedge d g_{n-1} \wedge d(\cdot)$ to $\tilde{f}$ and making use of the fact that $\tilde{f}$ is a Weierstrass polynomial of the type described above. To continue applying the operator $d g_{1} \wedge \cdots \wedge d g_{n-1} \wedge d(\cdot)$ to $\tilde{f}$, we need to modify first the result from the previous differentiation by comparing on $V$ the Jacobian determinant

$$
\frac{\partial\left(g_{1}, \cdots, g_{n-1}\right)}{\partial\left(z_{1}, \cdots, z_{n-1}\right)}
$$

with an appropriate polynomial $p\left(g_{1}, \cdots, g_{n-1}\right)$ of $g_{1}, \cdots, g_{n-1}$ and using the Real Radical Property of Kohn's algorithm in (I.1)(B) to replace

$$
\frac{\partial\left(g_{1}, \cdots, g_{n-1}\right)}{\partial\left(z_{1}, \cdots, z_{n-1}\right)}
$$

by $p\left(g_{1}, \cdots, g_{n-1}\right)$. The final result of differentiating $\tilde{f}$ this way as many times as the multiplicity of $\tilde{f}$ at the origin produces a new multiplier which defines on $V$ a subspace with effective multiplicity at the origin. This way of cutting down on the dimension of the subspace defined by such effectively constructed multipliers gives the effective termination of Kohn's algorithm for special domains.

In the details of the proof for special domains given below in (III.6), (III.7), (III.8), and (III.9), we actually do not carry out completely the induction of cutting down on the dimension of the zero-set of effectively constructed multipliers. A short-cut is used to simplify the process to reach the same goal (see (III.9)). 
(II.3) Modification for Effectiveness for Real-Analytic Case. Before we give the rigorous details of the proof of the effective termination of the Kohn algorithm for special domains, we would like to discuss how the techniques in the above sketch for special domains in (II.2) can be modified for the general real-analytic case. We consider the following real-analytic case where the weakly pseudoconvex domain of finite type is defined by $r<0$ with $r\left(z_{1}, \cdots, z_{n}, \overline{z_{1}}, \cdots, \overline{z_{n}}\right)$ being realanalytic and vanishing at the origin (which is the boundary point we consider). The main idea is to let $w_{j}=\overline{z_{j}}$ for $1 \leq j \leq n$ and let $R$ be the ring of convergent power series in $w_{1}, \cdots, w_{n}$ and consider the $n+1$ holomorphic function germs $H_{0}, H_{1}, \cdots, H_{n}$ on $\mathbb{C}^{n}$ (with coordinates $z_{1}, \cdots, z_{n}$ ) at the origin with coefficients in the ring $R$ defined as follows.

$$
\begin{gathered}
H_{0}\left(z_{1}, \cdots, z_{n}\right)=r\left(z_{1}, \cdots, z_{n}, w_{1}, \cdots, w_{n}\right), \\
H_{j}\left(z_{1}, \cdots, z_{n}\right)=\frac{\partial r}{\partial w_{j}}\left(z_{1}, \cdots, z_{n}, w_{1}, \cdots, w_{n}\right) \quad \text { for } 1 \leq j \leq n,
\end{gathered}
$$

where the coefficients of the power series expansion of $H_{j}$ in $z_{1}, \cdots, z_{n}$ are all elements of $R$ for $0 \leq j \leq n$. For the complex Euclidean space $\mathbb{C}^{n}$ with coordinates $z_{1}, \cdots, z_{n}$ we denote by $\mathfrak{m}_{\mathbb{C}^{n}, 0}$ the maximum ideal of $\mathbb{C}^{n}$ at the origin. Finite type condition for the domain $\{r<0\}$ at the origin implies the statement that

(II.3.1) there exists some effective positive integer $q$ such that $R\left(\mathfrak{m}_{\mathbb{C}^{n}, 0}\right)^{q}$ is contained in the ideal generated by $H_{0}, H_{1}, \cdots, H_{n}$ in the $\operatorname{ring} R\left\{z_{1}, \cdots, z_{n}\right\}$ of convergent power series in $z_{1}, \cdots, z_{n}$ with coefficients in $R$.

The statement (II.3.1) simply follows directly from the definition of finite type. It can be regarded as the real-analytic analog of condition (I.2.3) for a special domain. Note that finite type is actually much stronger than the statement (II.3.1).

In a way analogous to applying condition (I.2.3) to do an inductive multiplierconstruction process to obtain a nonzero constant as a multiplier from the Kohn algorithm for a special domain as described in (II.2), we now apply statement (II.3.1) to do the same inductive multiplier-construction process with the difference that now the coefficients of the power series of the function germs involved are elements of $R=\left\{w_{1}, \cdots, w_{n}\right\}$ instead of just $\mathbb{C}$. One modification is needed for the inductive multiplier-construction process. When we are in the case of a special domain, we use $n$ generic $\mathbb{C}$-linear combinations $g_{1}, \cdots, g_{n}$ of 
$F_{1}, \cdots, F_{N}$, but here in the real-analytic case when we choose $n R$-linear combinations $g_{1}, \cdots, g_{n}$ of $H_{0}, \cdots, H_{n}$, one of $g_{1}, \cdots, g_{n}$ must be chosen to be $H_{0}$. The reason for this modification is that we are not in the special case where the domain is of the form

$$
\operatorname{Re} w+r\left(z_{1}, \cdots, z_{n}, \overline{z_{1}}, \cdots, \overline{z_{n}}\right)<0
$$

and when our domain is not in this special form we have to use $\partial r$ to define the tangent space of type $(1,0)$ for the boundary of the domain.

Note that when we take $\partial G_{1} \wedge \cdots \wedge \partial G_{n-1} \wedge \partial H_{0}$ for generic $\mathbb{C}$-linear combinations $G_{1}, \cdots, G_{n-1}$ of $H_{1}, \cdots, H_{n}$, we are simply using (I.1)(A)(ii) and (I.1)(B)(ii) in Kohn's algorithm.

The inductive multiplier-construction process in the real-analytic case now gives us a nonzero element $f$ of $R$ instead of a nonzero element of $\mathbb{C}$ in the case of a special domain. The main point is that, because of the finite type condition the multiplicity of this element $f\left(w_{1}, \cdots, w_{n}\right)$ of $R$ at 0 is bounded effectively by a constant depending on $n$ and the order of the finite type. Now we consider the anti-holomorphic function germ $\tilde{f}$ on $\mathbb{C}^{n}$ at the origin defined by $\tilde{f}=f\left(\overline{z_{1}}, \cdots, \overline{z_{n}}\right)$ and consider the complex conjugate $g$ of $\tilde{f}$.

We let $V_{1}$ be the subspace germ defined by the holomorphic function germ $g$ on $\mathbb{C}^{n}$ at the origin. We then consider $V_{1} \times \overline{V_{1}}$ in $\mathbb{C}^{n} \times \mathbb{C}^{n}$ instead of the full $2 n$ dimensional complex Euclidean space $\mathbb{C}^{n} \times \mathbb{C}^{n}$ itself (with $z_{1}, \cdots, z_{n}, \overline{z_{1}}, \cdots, \overline{z_{n}}$ being the variables of $\left.\mathbb{C}^{n} \times \mathbb{C}^{n}\right)$. Let $R_{1}$ be the ring of holomorphic function germs on $V_{1}$ at 0 when $V_{1}$ is considered as a subspace germ of 0 in $\mathbb{C}^{n}$ at the origin with coordinates $w_{1}, \cdots, w_{n}$. We now apply the inductive process to obtain a holomorphic function germ $f_{1}$ on $V_{1}$ at 0 (which is a subspace germ at 0 of $\mathbb{C}^{n}$ with variables $\left.w_{1}, \cdots, w_{n}\right)$.

Now we consider the function germ $\tilde{f}_{1}$ obtained from $f_{1}$ by replacing $w_{1}, \cdots, w_{n}$ by $\overline{z_{1}}, \cdots, \overline{z_{n}}$. Let $g_{1}$ be the complex-conjugate of $\tilde{f}_{1}$. Let $V_{2}$ be a complete intersection of codimension two in $\mathbb{C}^{n}$ at the origin defined by two holomorphic functions which belong to the radical of the ideal generated by $g_{2}$ and the ideal of $V_{1}$. We then consider $V_{2} \times \overline{V_{2}}$ in $\mathbb{C}^{n} \times \mathbb{C}^{n}$ instead of $\mathbb{C}^{n} \times \mathbb{C}^{n}$ itself (with $z_{1}, \cdots, z_{n}, \overline{z_{1}}, \cdots, \overline{z_{n}}$ being the variables of $\left.\mathbb{C}^{n} \times \mathbb{C}^{n}\right)$. Let $R_{2}$ be the ring of holomorphic function germs on $V_{2}$ at 0 when $V_{2}$ is considered as a subspace germ of 0 in $\mathbb{C}^{n}$ with coordinates $w_{1}, \cdots, w_{n}$. We now can continue with this 
inductive subspace-construction process which so far yields for us the subspace of complete intersection $V_{1}$ and $V_{2}$. We continue with this inductive subspaceconstruction process to get $V_{\ell+1}$ from $V_{\ell}$ for $1 \leq \ell \leq n-1$ until we get to the subspace $V_{n}$ of $\mathbb{C}^{n}$ which consists only of the origin. This then immediately gives us the effective termination of Kohn's algorithm for the real-analytic case. Again, instead of carrying out completely the inductive argument of cutting down the dimension of the subspace described above, it is also possible to use the analog of the short-cut technique given in (III.9).

Another way of describing this modification is to redo the algebraic-geometric argument for the case of a special domain but to do it over a parameter space defined by the ring $R$. The coordinates for $R$ are the complex-conjugates of the coordinates for the ambient space $\mathbb{C}^{n}$. We can describe the modification as redoing the algebraic-geometric argument for the case of a special domain over $\operatorname{Spec}(R)$ instead of over the single point $\operatorname{Spec}(\mathbb{C})$. While the case of a special domain yields effectively a nonzero element of $\mathbb{C}$ as a multiplier, the real-analytic case would yield effectively a nonzero element of $R$. Then we replace $\mathbb{C}^{n}$ or by the subspace defined by this nonzero element of $R$ and repeat the argument to get down to lower and lower dimensional subspaces until we get to a single point, or we use the analog of the short-cut technique given in (III.9).

(II.4) Modification for Effectiveness for Smooth Case. We are going to have yet another discussion, this time about modifying further the techniques in the above sketch for special domains in (II.2) in order to handle the general smooth case, before going into the rigorous details of the proof of the effective termination of the Kohn algorithm for special domains. Now suppose that we have a smooth bounded weakly pseudoconvex domain $\Omega$ of finite type given by $r<0$ for some smooth function $r$ defined on some neighborhood of the topological closure $\bar{\Omega}$ of $\Omega$ in $\mathbb{C}^{n}$ and that the origin 0 of $\mathbb{C}^{n}$ is a boundary point of $\Omega$.

Let $q$ be the positive integer which is the order of the finite type of the origin as a boundary point of $\Omega$. Let $r_{N}$ be the $N$-th partial sum of the formal power series expansion of $r$ at the origin with respect to the coordinates $z_{1}, \cdots, z_{n}$ of $\mathbb{C}^{n}$. We choose $N$ effectively large enough so that the type of $r_{N}=0$ at the origin is also $q$. 
We apply Kohn's algorithm for the real-analytic case to $r_{N}$. From the effectiveness for the real-analytic case (II.3), we can find some positive integer $N_{q}$ which depends only on $q$ and $n$ such that the assigned order of subellipticity $\varepsilon$ for the final nonzero constant multiplier from the effective Kohn algorithm for $r_{N}$ satisfies $\varepsilon>\frac{1}{N_{q}}$.

When we choose $N$ effectively large enough, for example, $N>2 N_{p}$, the effective termination of Kohn's algorithm for $r_{N}$ also gives the effective termination of Kohn's algorithm for $r$ with precisely the same steps and the same assigned order of subellipticity for each step. Note that this process of approximating $r$ by $r_{N}$ is very different from the approximation of a bounded smooth weakly pseudoconvex domain of finite type by a real-analytic smooth weakly pseudoconvex domain of finite type, which is in general not possible. The $N$-th partial sum $r_{N}$ is simply used as an algebraic-geometric comparison guide to guarantee the effective termination of Kohn's algorithm for the original smooth defining function $r$.

Note that when we do the approximation of $r$ by $r_{N}$, we are doing this approximation only at the boundary point under consideration and not using the approximation along the normal directions of the zero-sets of multipliers from Kohn's algorithm for $r$. The reason is that the purpose of the approximation is to use the effective termination of Kohn's algorithm for the real-analytic function $r_{N}$ to conclude for a sufficiently large $N$ that the corresponding steps result in the effective termination of Kohn's algorithm for the smooth function $r$. The motivation for choosing this procedure of approximation is twofold. One is that the notion of finite type at a boundary point of the weakly pseudoconvex domain depending only on the formal power series expansion of the defining function $r$ at that point. The other is that the zero-sets of multipliers from Kohn's algorithm for $r$ are defined by the vanishing of smooth functions and it is not clear how one can do a real-analytic approximation along the normal directions of such zero-sets. In our use of the approximation of $r$ by $r_{N}$, the zero-sets of multipliers from Kohn's algorithm for $r$ are different from the zero-sets of multipliers from Kohn's algorithm for $r_{N}$. When we use the "real radical property" to produce multipliers from Kohn's algorithm for $r_{N}$, we simply perform the same operation for the corresponding but different zero-set in Kohn's algorithm for $r$. 


\section{Part III. Details of Proof of Effective Termination of Kohn's Algorithm for Special Domains}

(III.1) Precise Formulation. Let $F_{1}, \cdots, F_{N}$ be holomorphic function germs on $\mathbb{C}^{n}$ at the origin 0 . Assume that

$$
s:=\operatorname{dim}_{\mathbb{C}}\left(\mathcal{O}_{\mathbb{C}^{n}, 0} / \sum_{j=1}^{N} \mathcal{O}_{\mathbb{C}^{n}, 0} F_{j}\right)<\infty
$$

so that the subscheme of $\mathbb{C}^{n}$ defined by $F_{1}, \cdots, F_{N}$ is an Artinian subscheme. We will call $s$ the multiplicity of the ideal generated by $F_{1}, \cdots, F_{N}$. This definition agrees with that given in (III.3) below for ideals generated by $k$ holomorphic function germs whose common zero-set is of complex codimension $k$. Let

$$
\mathcal{A}_{1}=\sum_{j=1}^{N} \mathcal{O}_{\mathbb{C}^{n}, 0}\left(d F_{j}\right)
$$

be the $\mathcal{O}_{\mathbb{C}^{n}, 0}$-submodule of the $\mathcal{O}_{\mathbb{C}^{n}, 0}$-module $\mathcal{O}_{\mathbb{C}^{n}, 0}\left(T_{\mathbb{C}^{n}, 0}\right)^{*}$ of all germs of holomorphic $(1,0)$-forms on $\mathbb{C}^{n}$ at 0 . Take a sequence of positive integers $q_{\nu}$ for any positive integer $\nu$. By induction on the positive integer $\nu$ we define as follows the ideals $\mathcal{I}_{\nu}$ and $\mathcal{J}_{\nu}$ of $\mathcal{O}_{\mathbb{C}^{n}, 0}$ and the $\mathcal{O}_{\mathbb{C}^{n}, 0^{-}}$-submodule $\mathcal{A}_{\nu+1}$ of the $\mathcal{O}_{\mathbb{C}^{n}, 0^{-}}$-module $\mathcal{O}_{\mathbb{C}^{n}, 0}\left(T_{\mathbb{C}^{n}, 0}\right)^{*}$.

For $\nu \geq 1$ the ideal $\mathcal{J}_{\nu}$ of $\mathcal{O}_{\mathbb{C}^{n}, 0}$ is generated over $\mathcal{O}_{\mathbb{C}^{n}, 0}$ by all holomorphic function-germs $f$ on $\mathbb{C}^{n}$ at 0 satisfying

$$
g_{1} \wedge \cdots \wedge g_{n}=f\left(d z_{1} \wedge \cdots \wedge d z_{n}\right)
$$

with $g_{1}, \cdots, g_{n} \in \mathcal{A}_{\nu}$. The ideal $\mathcal{I}_{\nu}$ is defined by the set of all holomorphic function germs $f$ on $\mathbb{C}^{n}$ at 0 so that $f^{q} \in \mathcal{J}_{\nu}$ for some $1 \leq q \leq q_{\nu}$.

For $\nu \geq 2$ the $\mathcal{O}_{\mathbb{C}^{n}, 0}$-submodule $\mathcal{A}_{\nu}$ of the $\mathcal{O}_{\mathbb{C}^{n}, 0}$-module $\mathcal{O}_{\mathbb{C}^{n}, 0}\left(T_{\mathbb{C}^{n}, 0}\right)^{*}$ is generated by all $d f$ for $f \in \mathcal{I}_{\nu-1}$ and all elements of $\mathcal{A}_{\nu-1}$.

(III.2) Main Theorem. There exists an explicit sequence $\left\{q_{\nu}\right\}_{\nu \in \mathbb{N}}$ and an explicit number $m$ depending only on $n$ and $s$ such that $\mathcal{I}_{m}=\mathcal{O}_{\mathbb{C}^{n}, 0}$.

To prepare for the proof of the Main Theorem, we put together some lemmas about selecting $\mathbb{C}$-linear combinations of $F_{1}, \cdots, F_{N}$ to generate ideals with effective multiplicity and about estimating the multiplicity of Jacobian determinants. 
(III.3) Lemma (on Selection of Linear Combinations of Holomorphic Functions for Effective Multiplicity). Let $0 \leq q \leq n$. Let $f_{1}, \cdots, f_{q}$ be holomorphic function germs on $\mathbb{C}^{n}$ at the origin whose common zero-set $W_{q}$ is of pure codimension $q$ in $\mathbb{C}^{n}$ as a subvariety germ, with the convention that $W_{0}=\mathbb{C}^{n}$ and $\sum_{j=1}^{0} \mathcal{O}_{\mathbb{C}^{n}, 0} f_{j}=$ 0 for the case $q=0$. Let $m$ be the multiplicity of the ideal $\sum_{j=1}^{q} \mathcal{O}_{\mathbb{C}^{n}, 0} f_{j}$ at the origin in the sense that

$$
\operatorname{dim}_{\mathbb{C}}\left(\mathcal{O}_{\mathbb{C}^{n}, 0} /\left(\sum_{j=1}^{q} \mathcal{O}_{\mathbb{C}^{n}, 0} f_{j}+\sum_{j=1}^{n-q} \mathcal{O}_{\mathbb{C}^{n}, 0} L_{j}\right)\right)=m
$$

for any $n-q$ generic $\mathbb{C}$-linear functions $L_{1}, \cdots, L_{n-q}$ on $\mathbb{C}^{n}$. Let $F_{j}\left(z_{1}, \cdots, z_{n}\right)$ $(1 \leq j \leq N)$ be holomorphic function germs on $\mathbb{C}^{n}$ at the origin which vanish at the origin. Let $p$ be a positive integer and $A$ be a positive number such that

$$
|z|^{p} \leq A \sum_{j=1}^{N}\left|F_{j}(z)\right|
$$

for all $z$ in the domain of definition of $F_{j}\left(z_{1}, \cdots, z_{n}\right)(1 \leq j \leq N)$. Then for generic choices of complex numbers

$$
\left\{c_{j, k}\right\}_{1 \leq j \leq n-q, 1 \leq k \leq N}
$$

the $\mathbb{C}$-linear combinations

$$
\tilde{F}_{j}=\sum_{k=1}^{N} c_{j, k} F_{k} \quad(1 \leq j \leq n-q)
$$

of $F_{1}, \cdots, F_{N}$ satisfy the property that

$$
\operatorname{dim}_{\mathbb{C}}\left(\mathcal{O}_{\mathbb{C}^{n}, 0} /\left(\sum_{j=1}^{q} \mathcal{O}_{\mathbb{C}^{n}, 0} f_{j}+\sum_{j=1}^{n-q} \mathcal{O}_{\mathbb{C}^{n}, 0} \tilde{F}_{j}\right)\right) \leq m p^{n-q} .
$$

That is, the multiplicity of the ideal generated by $f_{1}, \cdots, f_{q}, \tilde{F}_{1} \cdots, \tilde{F}_{n-q-1}$ is $\leq m p^{n-q}$ at the origin.

Proof. We use induction on $1 \leq \nu \leq n-q$ to show that for generic complex numbers

$$
\left\{c_{j, k}\right\}_{1 \leq j \leq \nu, 1 \leq k \leq N}
$$


the dimension at the origin of the common zero-set $V_{\nu}$ of the $f_{1}, \cdots, f_{q}$ and the $\mathbb{C}$-linear combinations

$$
\tilde{F}_{j}=\sum_{k=1}^{N} c_{j, k} F_{k} \quad(1 \leq j \leq \nu)
$$

of $F_{1}, \cdots, F_{N}$ is precisely $n-q-\nu$ and the multiplicity of the ideal

$$
\sum_{j=1}^{q} \mathcal{O}_{\mathbb{C}^{n}, 0} f_{j}+\sum_{j=1}^{\nu} \mathcal{O}_{\mathbb{C}^{n}, 0} \tilde{F}_{j}
$$

is no more than $m p^{\nu}$.

We introduce the case of $\nu=0$ and the convention that $\sum_{j=1}^{0} \mathcal{O}_{\mathbb{C}^{n}, 0} \tilde{F}_{j}=0$ for the case $\nu=0$. With this convention, we start out our induction assumption with the case $\nu=0$ which is trivially true.

Suppose the induction process has been carried out for some $0 \leq \nu<n-q$ and we would like to verify it for the next step when $\nu$ is replaced by $\nu+1$. We now already have $\tilde{F}_{1}, \cdots, \tilde{F}_{\nu}$. Let

$$
\mathcal{I}_{\nu}=\sum_{j=1}^{q} \mathcal{O}_{\mathbb{C}^{n}} f_{j}+\sum_{j=1}^{\nu} \mathcal{O}_{\mathbb{C}^{n}} \tilde{F}_{j}
$$

The zero-set of $\mathcal{I}_{\nu}$ is the subvariety $V_{\nu}$ of pure dimension $n-q-\nu$. Let $E_{\nu}$ be a generic linear subspace of $\mathbb{C}^{n}$ of codimension $n-q-\nu-1$ defined by $n-q-\nu-1$ generic linear functions $G_{1}, \cdots, G_{n-q-\nu+1}$ so that the subvariety $V_{\nu} \cap E_{\nu}$ is of pure dimension 1 . Let

$$
\mathcal{J}_{\nu}=\sum_{j=1}^{n-q-\nu-1} \mathcal{O}_{\mathbb{C}^{n}} G_{j}+\sum_{j=1}^{q} \mathcal{O}_{\mathbb{C}^{n}} f_{j}+\sum_{j=1}^{\nu} \mathcal{O}_{\mathbb{C}^{n}} \tilde{F}_{j}
$$

Let

$$
\mathcal{J}_{\nu}=\bigcap_{\lambda=1}^{\Lambda} \mathcal{L}_{\lambda}
$$

be the primary decomposition of the ideal sheaf $\mathcal{J}_{\nu}$. Note that, since the zero-set of $\mathcal{J}_{\nu}$ is of pure complex dimension 1 and $\mathcal{J}_{\nu}$ is generated by $n-1$ holomorphic functions

$$
G_{1}, \cdots, G_{n-q-\nu+1}, f_{1}, \cdots, f_{q}, \tilde{F}_{1}, \cdots, \tilde{F}_{\nu},
$$

it follows that all the associated prime ideals of $\mathcal{J}_{\nu}$ are isolated and none are embedded [ZS60, p.397, Theorem 2]. 
Let $C_{\lambda}$ be the complex curve-germ which is the zero-set of the ideal sheaf $\mathcal{L}_{\lambda}$. Let $\mu_{\lambda}$ be the multiplicity of the curve $C_{\lambda}$ at the origin. Let $\hat{\mu}_{\lambda}$ be the multiplicity of the ideal sheaf $\mathcal{L}_{\lambda}$ at a generic point $Q \in C_{\lambda}$, which can be characterized as the dimension over $\mathbb{C}$ of

$$
\mathcal{O}_{\mathbb{C}^{n}, Q} /\left(\left(\mathcal{L}_{\lambda}\right)_{Q}+\mathcal{O}_{\mathbb{C}^{n}, Q} L\right)
$$

where $L$ is a generic polynomial of degree 1 on $\mathbb{C}^{n}$ vanishing at $Q$ and $\left(\mathcal{L}_{\lambda}\right)_{Q}$ is the stalk of the ideal sheaf $\mathcal{L}_{\lambda}$ at the point $Q$.

Without loss of generality we can assume that the coordinates $\left(z_{1}, \cdots, z_{n}\right)$ of $\mathbb{C}^{n}$ are chosen so that $C_{\lambda}$ is defined by

$$
\left\{\begin{array}{l}
z_{1}=\zeta^{\mu_{\lambda}}, \\
z_{j}=g_{\lambda, j}(\zeta) \text { for } 2 \leq j \leq n
\end{array}\right.
$$

for $\zeta$ in some open neighborhood of the origin in $\mathbb{C}$, where the initial term of $g_{\lambda, j}(\zeta)$ is a nonzero complex number times $\zeta^{N_{\lambda, j}}$ for some $N_{\lambda, j} \geq \mu_{\lambda}$ for $2 \leq j \leq n$. Let $\pi_{\lambda}: \tilde{C}_{\lambda} \rightarrow C_{\lambda}$ be the normalization of $C_{\lambda}$ defined by

$$
\pi_{\lambda}: \zeta \mapsto z=\left(\zeta^{\mu_{\lambda}}, g_{\lambda, 2}(\zeta), \cdots, g_{\lambda, n}(\zeta)\right)
$$

where $\tilde{C}_{\lambda}$ is an open neighborhood of 0 in $\mathbb{C}$ with $\zeta$ as coordinate. The pullback $\pi_{\lambda}^{*} \mathfrak{m}_{C_{\lambda}, 0}$ to $\tilde{C}_{\lambda}$ of the maximum ideal $\mathfrak{m}_{C_{\lambda}, 0}$ of $C_{\lambda}$ at the origin is generated by $\zeta^{\mu_{\lambda}}, g_{\lambda, 2}(\zeta), \cdots, g_{\lambda, n}(\zeta)$. Since $\pi_{\lambda}^{*} \mathfrak{m}_{C_{\lambda}, 0}$ is a principal ideal, it must be generated by $\zeta^{\mu_{\lambda}}$.

The inequality (III.3.1), when pulled back by $\pi_{\lambda}$, becomes

$$
|\zeta|^{p \mu_{\lambda}} \leq A_{\lambda} \sum_{j=1}^{N}\left|\left(F_{j} \circ \pi_{\lambda}(\zeta)\right)\right|
$$

for $1 \leq \lambda \leq \Lambda$, where $A_{\lambda}$ is a positive number. Take a generic point

$$
\left(c_{\nu+1,1}, \cdots, c_{\nu+1, N}\right) \in \mathbb{C}^{N}
$$

and let

$$
\tilde{F}_{\nu+1}=\sum_{k=1}^{N} c_{\nu+1, k} F_{k} .
$$

By (III.3.2 $)_{\nu}$, for each $1 \leq \lambda \leq \Lambda$ the vanishing order of $\left(\tilde{F}_{\nu+1} \circ \pi_{\lambda}\right)(\zeta)$ at $\zeta=0$ is some number $\tilde{\mu}_{\lambda}$ which is no more than $p \mu_{\lambda}$. For a small generic nonzero $\eta \in \mathbb{C}$ the number of zeros of $\eta+\left(\tilde{F}_{\nu+1} \circ \pi_{\lambda}\right)(\zeta)$ on $\tilde{C}_{\lambda}$ is precisely $\tilde{\mu}_{\lambda}$ with multiplicity 
1 for each $1 \leq \lambda \leq \Lambda$. Since the map $\pi_{\lambda}: \tilde{C}_{\lambda} \rightarrow C_{\lambda}$ is one-to-one, it follows that for any small generic nonzero $\eta \in \mathbb{C}$ the number of zeroes of $\eta+\tilde{F}_{\nu+1}$ on $C_{\lambda}$ is precisely $\tilde{\mu}_{\lambda}$ with multiplicity 1 .

Since the multiplicity of the ideal sheaf $\mathcal{L}_{\lambda}$ at a generic point $Q \in C_{\lambda}$ is $\hat{\mu}_{\lambda}$, it follows from $\tilde{\mu}_{\lambda} \leq p \mu_{\lambda}$ that the dimension over $\mathbb{C}$ of the vector space

$$
\mathcal{O}_{\mathbb{C}^{n}, 0} /\left(\sum_{j=1}^{n-q-\nu-1} \mathcal{O}_{\mathbb{C}^{n}, 0} G_{j}+\sum_{j=1}^{q} \mathcal{O}_{\mathbb{C}^{n}, 0} f_{j}+\sum_{j=1}^{\nu+1} \mathcal{O}_{\mathbb{C}^{n}, 0} \tilde{F}_{j}\right)
$$

is no more than $p \sum_{\lambda=1}^{\Lambda} \mu_{\lambda} \hat{\mu}_{\lambda}$. By induction hypothesis the multiplicity of

$$
\mathcal{I}_{\nu}=\sum_{j=1}^{q} \mathcal{O}_{\mathbb{C}^{n}} f_{j}+\sum_{j=1}^{\nu} \mathcal{O}_{\mathbb{C}^{n}} \tilde{F}_{j}
$$

is no more than $m p^{\nu}$ at the origin. The multiplicity of $\mathcal{J}_{\nu}$ at the origin, which can be computed from $\mathcal{I}_{\nu}$ by adding generic $\mathbb{C}$-linear functions $G_{1}, \cdots, G_{n-q-\nu-1}$ on $\mathbb{C}^{n}$, is also no more than $m p^{\nu}$. We can compute the multiplicity of $\mathcal{J}_{\nu}$ at the origin by adding to $\mathcal{J}_{\nu}$ a generic $\mathbb{C}$-linear function $L$ on $\mathbb{C}^{n}$ and considering the sum of the multiplicities at points of intersection of the zero-set with $L+\eta$ for some small generic $\eta \in \mathbb{C}$. From the decomposition $\mathcal{J}_{\nu}=\bigcap_{\lambda=1}^{\Lambda} \mathcal{L}_{\lambda}$ and the multiplicity $\hat{\mu}_{\lambda}$ of $\mathcal{L}_{\lambda}$ at the origin we conclude that

$$
\sum_{\lambda=1}^{\Lambda} \mu_{\lambda} \hat{\mu}_{\lambda} \leq m p^{\nu}
$$

Thus

$$
\operatorname{dim}_{\mathbb{C}}\left(\mathcal{O}_{\mathbb{C}^{n}, 0} /\left(\sum_{j=1}^{n-q-\nu+1} \mathcal{O}_{\mathbb{C}^{n}, 0} G_{j}+\sum_{j=1}^{q} \mathcal{O}_{\mathbb{C}^{n}, 0} f_{j}+\sum_{j=1}^{\nu+1} \mathcal{O}_{\mathbb{C}^{n}, 0} \tilde{F}_{j}\right)\right) \leq m p^{\nu+1}
$$

Since $G_{1}, \cdots, G_{n-q-\nu+1}$ are generic linear functions on $\mathbb{C}^{n}$, it follows that the multiplicity of the ideal

$$
\sum_{j=1}^{q} \mathcal{O}_{\mathbb{C}^{n}, 0} f_{j}+\sum_{j=1}^{\nu+1} \mathcal{O}_{\mathbb{C}^{n}, 0} \tilde{F}_{j}
$$

at the origin is no more than $m p^{\nu+1}$. This finishes the induction process. Q.E.D. (III.4) Corollary. Let $F_{j}\left(z_{1}, \cdots, z_{n}\right)(1 \leq j \leq N)$ be holomorphic function germs on $\mathbb{C}^{n}$ at the origin which vanish at the origin. Let $p$ be a positive integer and $A$ 
be a positive number such that

$$
|z|^{p} \leq A \sum_{j=1}^{N}\left|F_{j}(z)\right|
$$

for all $z$ in the domain of definition of $F_{j}\left(z_{1}, \cdots, z_{n}\right)(1 \leq j \leq N)$. Then for generic choices of complex numbers

$$
\left\{c_{j, k}\right\}_{1 \leq j \leq n, 1 \leq k \leq N}
$$

the $\mathbb{C}$-linear combinations

$$
\tilde{F}_{j}=\sum_{k=1}^{N} c_{j, k} F_{k} \quad(1 \leq j \leq n)
$$

of $F_{1}, \cdots, F_{N}$ satisfy the property that

$$
\operatorname{dim}_{\mathbb{C}}\left(\mathcal{O}_{\mathbb{C}^{n}, 0} / \sum_{j=1}^{n} \mathcal{O}_{\mathbb{C}^{n}, 0} \tilde{F}_{j}\right) \leq p^{n} .
$$

Proof. Introduce one more complex variable $w$ and consider $F_{j}$ as a holomorphic function germ on $\mathbb{C}^{n+1}$ at 0 in the variables $z_{1}, \cdots, z_{n}, w$ though it is independent of the variable $w$. Add the function $w$ to the functions $F_{1}, \cdots, F_{N}$. Let $f_{1}=w$ and apply Lemma (III.3) on Selection of Linear Combinations of Holomorphic Functions for Effective Multiplicity to the case $m=1$ with $\mathbb{C}^{n}$ replaced by $\mathbb{C}^{n+1}$. Q.E.D.

(III.5) Lemma (Multiplicity Estimate for Jacobian Determinant). Let $g_{1}, \cdots, g_{n}$ be holomorphic function germs on $\mathbb{C}^{n}$ at the origin such that

$$
\operatorname{dim}_{\mathbb{C}}\left(\mathcal{O}_{\mathbb{C}^{n}, 0} / \sum_{j=1}^{n} \mathcal{O}_{\mathbb{C}^{n}, 0} g_{j}\right) \leq m .
$$

Let $d g_{1} \wedge \cdots \wedge d g_{n}=f\left(d z_{1}, \cdots, z_{n}\right)$. Then the multiplicity of $f$ at the origin is $\leq m$.

Proof. We can find a connected open neighborhood $U$ of 0 in $\mathbb{C}^{n}$ and an open ball neighborhood $W$ of 0 in $\mathbb{C}^{n}$ such that the map $\pi: U \rightarrow W$ defined by $g_{1}, \cdots, g_{n}$ is a proper holomorphic map. This is possible, because the common zero-set of $g_{1}, \cdots, g_{n}$ consists only of the origin in a sufficiently small neighborhood of the origin in $\mathbb{C}^{n}$. The number of sheets in the analytic cover map $\pi: U \rightarrow V$ is $\leq m$. Let $Y$ be the divisor of $f$ in $U$ and $Z$ be the image of $Y$ in $W$. Let $Z_{0}$ be the 
set of regular points of (the reduction of $Z$ ). Let $L$ be a generic complex line in the target space $\mathbb{C}^{n}$ such that $L \cap Z$ is a single point $P$ in $Z_{0}$ and $L$ intersects $Z_{0}$ transversally at $P$. For a sufficiently small neighborhood $D$ of $P$ in $W$ the map $U \cap \pi^{-1}(D) \rightarrow D$ induced by $\pi$ is just a cyclic branched cover on each topological component of $U \cap \pi^{-1}(D)$. Thus the multiplicity of the intersection of the regular curve $\pi^{-1}(L)$ and the divisor $Y$ is no more than the number of sheets of $\pi: U \rightarrow W$. Since the line $L$ is generic, it follows that the multiplicity of the divisor $Y$ is more than $m$. Q.E.D.

(III.6) Preparatory Remarks on Proof of Main Theorem. We now start the setting for the proof of the Main Theorem (III.2). Let $F_{1}, \cdots, F_{N}$ be holomorphic function germs on $\mathbb{C}^{n}$ at the origin whose common zero-set is the origin. Let $q$ be a positive integer. Assume that, for some positive number $A$,

$$
|z|^{q} \leq A \sum_{j=1}^{N}\left|F_{j}(z)\right|
$$

for all $z$ in the domain of definition of $F_{j}\left(z_{1}, \cdots, z_{n}\right)(1 \leq j \leq N)$. Because of the discussion in (II.1), for the case of special domains we need only consider multipliers which are holomorphic and we need only consider vector-multipliers which are holomorphic $(1,0)$-forms. Though $F_{1}, \cdots, F_{N}$ are not multipliers, their differentials $d F_{1}, \cdots, d F_{N}$ are vector-multipliers and, in order to form Jacobian determinants to generate multipliers, we can also use $\ell \mathbb{C}$-linear combinations of $F_{1}, \cdots, F_{N}$ and $n-\ell$ multipliers for $0 \leq \ell \leq n$ to form a Jacobian determinant which will then be a multiplier. We will refer to any $\mathbb{C}$-linear combination of $F_{1}, \cdots, F_{N}$ and multipliers as pre-multipliers so that the $(1,0)$-differential of a pre-multiplier is a vector-multiplier. Note that the pre-multipliers form a $\mathbb{C}$-vector space but do not form an ideal. The product of a multiplier and a holomorphic function germ is again a multiplier, but the product of a pre-multiplier and a holomorphic function germ in general is not a pre-multiplier. In our proof of the Main Theorem (III.2) we will not use vector-multipliers, because we will directly form the Jacobian determinants of the holomorphic pre-multipliers to generate new multipliers to bypass the process of forming vector-multipliers by differentiation and then using Cramer's rule.

In order not to be encumbered by complicated expressions of constants, we will not explicitly keep track of the various effective bounds occurring in the proof. We introduce the following terminology. A multiplier is called effectively constructed 
if there is an effective upper bound for its multiplicity and there is an effective positive lower bound for its assigned order of subellipticity. Effective means some explicit function of the multiplicity of the ideal generated by the pre-multipliers $F_{1}, \cdots, F_{N}$, which means an explicit function of $q$ given in (III.6.1). The goal is to show that the function-germ with constant value 1 can be effectively constructed.

To make the argument more transparent and to minimize notational clutters, we start out with the proof of the simple case where $n=2$.

(III.7) Proof of Main Theorem for Dimension Two. We now assume that $n=2$ and we have holomorphic function germs $F_{1}, \cdots, F_{N}$ on $\mathbb{C}^{2}$ at the origin whose zero-set is the origin of $\mathbb{C}^{n}$. The multiplicity of the ideal generated by $F_{1}, \cdots, F_{N}$ is the number used to express effectiveness. By applying Corollary (III.4) and (III.5) to get two $\mathbb{C}$-linear combinations of $F_{1}, \cdots, F_{N}$ and form their Jacobian determinant, we get an effectively constructed multiplier $\tilde{h}_{2}\left(z_{1}, z_{2}\right)$ at the origin, which vanishes at the origin. Because the multiplicity of $\tilde{h}_{2}\left(z_{1}, z_{2}\right)$ is effectively bounded at the origin, by replacing $\tilde{h}_{2}\left(z_{1}, z_{2}\right)$ by the product of holomorphic function germs defining the branch germs of the reduction of the subspace defined by $\tilde{h}_{2}\left(z_{1}, z_{2}\right)$, we can assume without loss of generality that the subspace germ $C_{2}$ defined by $\tilde{h}_{2}$ is a reduced curve germ in $\mathbb{C}^{2}$ at the origin with effectively bounded multiplicity. Note that in general the curve germ $C_{2}$ is not irreducible, though $C_{2}$ is a reduced curve. A reduced curve means that its structure sheaf does not contain any nonzero nilpotent elements. For example, it means that $h_{2}$ does not vanish to order higher than one at any regular point of $C_{2}$.

Now the ideal generated by the functions $\frac{\partial F_{i}}{\partial z_{j}}$ for $1 \leq i \leq N$ and $j=1,2$ has effectively bounded multiplicity at the origin, because by Proposition(A.2) in Appendix A, for each fixed $1 \leq i \leq N$, the function germ $\left(F_{i}\right)^{3}$ at the origin belongs to the ideal generated by $\frac{\partial F_{i}}{\partial z_{j}}$ for $j=1,2$. We consider the pre-multiplier $h_{1}=\sum_{j=1}^{N} c_{j} F_{j}$ with generic $c_{j} \in \mathbb{C}$ for $1 \leq j \leq N$ and consider a new generic linear coordinate system $\left(w_{1}, w_{2}\right)$ which is related to $\left(z_{1}, z_{2}\right)$ by $w_{i}=\sum_{j=1}^{2} b_{i j} z_{j}$ with generic $b_{i j} \in \mathbb{C}$ for $1 \leq i, j \leq 2$. By (III.3) we can find generic $c_{j} \in \mathbb{C}$ for $1 \leq j \leq N$ and generic $b_{i j} \in \mathbb{C}$ for $1 \leq i, j \leq 2$ such that

(i) the ideal generated by $h_{1}$ and $\tilde{h}_{2}$ has effectively bounded multiplicity at the origin, 
(ii) the ideal generated by $\frac{\partial h_{1}}{\partial w_{1}}$ and $\tilde{h}_{2}$ has effectively bounded multiplicity at the origin, where the partial derivative $\frac{\partial h_{1}}{\partial w_{1}}$ is computed with $w_{2}$ being kept constant,

(iii) the projection $P \mapsto g(P)$ makes $C_{2}$ an analytic cover over $\mathbb{C}$ locally at the origin as germs, and

(iv) the projection $\left(w_{1}, w_{2}\right) \mapsto\left(h_{1}, w_{2}\right)$ makes $\mathbb{C}^{2}$ an analytic cover over $\mathbb{C}^{2}$ locally at the origin as germs.

Without loss of generality we can assume that the coordinate system $\left(w_{1}, w_{2}\right)$ is just the coordinate system $\left(z_{1}, z_{2}\right)$. Note that $h_{1}$ is only a pre-multiplier and in general may not be a multiplier. The function germ $\frac{\partial h_{1}}{\partial z_{1}}$ is in general not a multiplier and not even a pre-multiplier.

Consider the image $\hat{C}_{2}$ of $C_{2}$ under the projection $\left(z_{1}, z_{2}\right) \mapsto\left(h_{1}, z_{2}\right)$ and let

$$
h_{2}=z_{2}^{\lambda}+\sum_{j=0}^{\lambda-1} a_{j}\left(h_{1}\right) z_{2}^{j}
$$

be the Weierstrass polynomial in $\mathbb{C}^{2}$ with coordinates $\left(h_{1}, z_{2}\right)$ whose vanishing defines the curve-germ $\hat{C}_{2}$ at the origin in $\mathbb{C}^{2}$. This is possible, because the projection $P \mapsto h_{1}(P)$ makes $C_{2}$ an analytic cover over $\mathbb{C}$ locally at the origin as germs. When regarded as a function-germ in the variables $\left(z_{1}, z_{2}\right)$ the function-germ $h_{2}$ contains $\tilde{h}_{2}$ as a factor, because the inverse image of $\hat{C}_{2}$ under the projection $\left(z_{1}, z_{2}\right) \mapsto\left(h_{1}, z_{2}\right)$ contains $C_{2}$ and $C_{2}$ is a reduced curve. Since $\tilde{h}_{2}$ is a multiplier, it follows that $h_{2}$ is also a multiplier and is, in fact, an effectively constructed multiplier. The multipliers in the effective procedure presented here and also in (III.8) and (III.9) are all effectively constructed multipliers (unless explicitly pointed out otherwise) and we will drop the description "effectively constructed" when we mention these multipliers here and in (III.8) and (III.9). Sometimes, to highlight certain aspects of effectiveness, we may mention "the assigned order of subellipticity having an effective positive lower bound" or "the multiplicity having an effective upper bound" in conjunction with such multipliers, though according to the convention given here such multipliers are all effective constructed unless explicitly pointed out otherwise.

Since the ideal generated by $\frac{\partial h_{1}}{\partial z_{1}}$ and $\tilde{h}_{2}$ has effectively bounded multiplicity at the origin and since $h_{1}$ vanishes at the origin, it follows that, for some effectively bounded positive integer $s$, the function germ $\left(h_{1}\right)^{s}$ belongs to the ideal generated 
by $\frac{\partial h_{1}}{\partial z_{1}}$ and $\tilde{h}_{2}$. In particular,

$$
\left|\left(h_{1}\right)^{s}\right| \lesssim\left|\frac{\partial h_{1}}{\partial z_{1}}\right|+\left|\tilde{h}_{2}\right|
$$

Here and for the rest of this note the symbol $\lesssim$ means "less than some constant times" and is being used to avoid introducing new symbols for constants. We now form $d h_{1} \wedge d h_{2}$ and get

$$
\begin{gathered}
d h_{1} \wedge d h_{2}=d h_{1} \wedge\left(\lambda z_{2}^{\lambda-1} d z_{2}+\sum_{j=1}^{\lambda-1} j a_{j}\left(h_{1}\right) z_{2}^{j-1} d z_{2}+\sum_{j=0}^{\lambda-1} a_{j}^{\prime}\left(h_{1}\right) z_{2}^{j} d h_{1}\right) \\
=d h_{1} \wedge\left(\lambda z_{2}^{\lambda-1} d z_{2}+\sum_{j=1}^{\lambda-1} j a_{j}\left(h_{1}\right) z_{2}^{j-1} d z_{2}\right) \\
=\left(\frac{\partial h_{1}}{\partial z_{1}} d z_{1}+\frac{\partial h_{1}}{\partial z_{2}} d z_{2}\right) \wedge\left(\lambda z_{2}^{\lambda-1} d z_{2}+\sum_{j=1}^{\lambda-1} j a_{j}\left(h_{1}\right) z_{2}^{j-1} d z_{2}\right) \\
=\frac{\partial h_{1}}{\partial z_{1}}\left(\lambda z_{2}^{\lambda-1}+\sum_{j=1}^{\lambda-1} j a_{j}\left(h_{1}\right) z_{2}^{j-1}\right) d z_{1} \wedge d z_{2},
\end{gathered}
$$

where $a_{j}^{\prime}\left(h_{1}\right)$ is the derivative of $a_{j}\left(h_{1}\right)$ as a function of $h_{1}$. Since $h_{1}$ is a premultiplier, the coefficient of $d z_{1} \wedge d z_{2}$ in $d h_{1} \wedge d h_{2}$ is a multiplier. Thus

$$
\frac{\partial h_{1}}{\partial z_{1}}\left(\lambda z_{2}^{\lambda-1}+\sum_{j=1}^{\lambda-1} j a_{j}\left(h_{1}\right) z_{2}^{j-1}\right)
$$

is a multiplier. Since $\tilde{h}_{2}$ is a multiplier, it follows that

$$
\tilde{h}_{2}\left(\lambda z_{2}^{\lambda-1}+\sum_{j=1}^{\lambda-1} j a_{j}\left(h_{1}\right) z_{2}^{j-1}\right)
$$

is a multiplier. From (III.7.1) it follows that

$$
\begin{gathered}
\left|\left(h_{1}\right)^{s}\left(\lambda z_{2}^{\lambda-1}+\sum_{j=1}^{\lambda-1} j a_{j}\left(h_{1}\right) z_{2}^{j-1}\right)\right| \\
\lesssim\left|\frac{\partial h_{1}}{\partial z_{1}}\left(\lambda z_{2}^{\lambda-1}+\sum_{j=1}^{\lambda-1} j a_{j}\left(h_{1}\right) z_{2}^{j-1}\right)\right|+\left|\tilde{h}_{2}\left(\lambda z_{2}^{\lambda-1}+\sum_{j=1}^{\lambda-1} j a_{j}\left(h_{1}\right) z_{2}^{j-1}\right)\right| .
\end{gathered}
$$


Hence

$$
\left(h_{1}\right)^{s}\left(\lambda z_{2}^{\lambda-1}+\sum_{j=1}^{\lambda-1} j a_{j}\left(h_{1}\right) z_{2}^{j-1}\right)
$$

is a multiplier. Let $h_{2}^{(0)}=h_{2}$ and

$$
h_{2}^{(1)}=\left(h_{1}\right)^{s}\left(\lambda z_{2}^{\lambda-1}+\sum_{j=1}^{\lambda-1} j a_{j}\left(h_{1}\right) z_{2}^{j-1}\right)
$$

and for $1<\nu \leq \lambda$ define

$$
h_{2}^{(\nu)}=\left(h_{1}\right)^{s \nu}\left(\frac{\lambda !}{(\lambda-\nu) !} z_{2}^{\lambda-\nu}+\sum_{j=\nu}^{\lambda-1} \frac{j !}{(j-\nu) !} a_{j}\left(h_{1}\right) z_{2}^{j-\nu}\right) .
$$

We are going to verify by induction on $\nu$ that $h_{2}^{(\nu)}$ is a multiplier. We know that both $h_{2}^{(0)}$ and $h_{2}^{(1)}$ are multipliers. Assume that we have already verified that $h_{2}^{(0)}, \cdots, h_{2}^{(\nu-1)}$ are multipliers. Then $d h_{1} \wedge d h_{2}^{(\nu-1)}$ is equal to

$$
\begin{aligned}
d h_{1} & \wedge d\left(\left(h_{1}\right)^{s(\nu-1)}\left(\frac{\lambda !}{(\lambda-\nu+1) !} z_{2}^{\lambda-\nu+1}+\sum_{j=\nu-1}^{\lambda-1} \frac{j !}{(j-\nu+1) !} a_{j}\left(h_{1}\right) z_{2}^{j-\nu+1}\right)\right) \\
& =d h_{1} \wedge\left(\left(h_{1}\right)^{s(\nu-1)}\left(\frac{\lambda !}{(\lambda-\nu) !} z_{2}^{\lambda-\nu}+\sum_{j=\nu}^{\lambda-1} \frac{j !}{(j-\nu) !} a_{j}\left(h_{1}\right) z_{2}^{j-\nu}\right) d z_{2}\right) \\
= & \frac{\partial h_{1}}{\partial z_{1}}\left(\left(h_{1}\right)^{s(\nu-1)}\left(\frac{\lambda !}{(\lambda-\nu) !} z_{2}^{\lambda-\nu}+\sum_{j=\nu}^{\lambda-1} \frac{j !}{(j-\nu) !} a_{j}\left(h_{1}\right) z_{2}^{j-\nu}\right)\right) d z_{1} \wedge d z_{2}
\end{aligned}
$$

Since the coefficient of $d z_{1} \wedge d z_{2}$ in $d h_{1} \wedge d h_{2}^{(\nu-1)}$ is a multiplier, it follows that

$$
\frac{\partial h_{1}}{\partial z_{1}}\left(\left(h_{1}\right)^{s(\nu-1)}\left(\frac{\lambda !}{(\lambda-\nu) !} z_{2}^{\lambda-\nu}+\sum_{j=\nu}^{\lambda-1} \frac{j !}{(j-\nu) !} a_{j}\left(h_{1}\right) z_{2}^{j-\nu}\right)\right)
$$

is a multiplier. Since $\tilde{h}_{2}$ is a multiplier, it follows that

$$
\tilde{h}_{2}\left(\left(h_{1}\right)^{s(\nu-1)}\left(\frac{\lambda !}{(\lambda-\nu) !} z_{2}^{\lambda-\nu}+\sum_{j=\nu}^{\lambda-1} \frac{j !}{(j-\nu) !} a_{j}\left(h_{1}\right) z_{2}^{j-\nu}\right)\right)
$$


is a multiplier. From (III.7.1) it follows that

$$
\begin{aligned}
& \left|\left(h_{1}\right)^{s}\left(\left(h_{1}\right)^{s(\nu-1)}\left(\frac{\lambda !}{(\lambda-\nu) !} z_{2}^{\lambda-\nu}+\sum_{j=\nu}^{\lambda-1} \frac{j !}{(j-\nu) !} a_{j}\left(h_{1}\right) z_{2}^{j-\nu}\right)\right)\right| \\
& \lesssim\left|\frac{\partial h_{1}}{\partial z_{1}}\left(\left(h_{1}\right)^{s(\nu-1)}\left(\frac{\lambda !}{(\lambda-\nu) !} z_{2}^{\lambda-\nu}+\sum_{j=\nu}^{\lambda-1} \frac{j !}{(j-\nu) !} a_{j}\left(h_{1}\right) z_{2}^{j-\nu}\right)\right)\right| \\
& +\left|\tilde{h}_{2}\left(\left(h_{1}\right)^{s(\nu-1)}\left(\frac{\lambda !}{(\lambda-\nu) !} z_{2}^{\lambda-\nu}+\sum_{j=\nu}^{\lambda-1} \frac{j !}{(j-\nu) !} a_{j}\left(h_{1}\right) z_{2}^{j-\nu}\right)\right)\right| .
\end{aligned}
$$

Hence

$$
\left(h_{1}\right)^{s}\left(\left(h_{1}\right)^{s(\nu-1)}\left(\frac{\lambda !}{(\lambda-\nu) !} z_{2}^{\lambda-\nu}+\sum_{j=\nu}^{\lambda-1} \frac{j !}{(j-\nu) !} a_{j}\left(h_{1}\right) z_{2}^{j-\nu}\right)\right)
$$

is a multiplier and $h_{2}^{(\nu)}$ is a multiplier. When $\nu=\lambda$, we end up with

$$
h_{2}^{(\lambda)}=\left(h_{1}\right)^{s \lambda} \lambda !
$$

being a multiplier.

Note that this step of forming Jacobian determinants $\lambda$ times to construct $h_{2}^{(\lambda)}$ from $\tilde{h}_{2}$ is the step of differentiating a multiplier as many times as its multiplicity to form a new multiplier, which is referred to at the end of the Introduction of this note. Also note that though this step only requires $h_{2}$ to be a premultiplier, yet $\tilde{h}_{2}$ has to be a multiplier instead of just a pre-multiplier, otherwise we cannot conclude that $h_{2}$ is a pre-multiplier, because in general the set of all pre-multipliers do not form an ideal.

Since the multiplicity of the ideal generated by $h_{1}$ and $\tilde{h}_{2}$ is effectively bounded, there exists some positive integer $\sigma$ which is effectively bounded such that $z_{1}^{\sigma}$ and $z_{2}^{\sigma}$ both belong to the ideal generated by $h_{2}^{(\lambda)}$ and $\tilde{h}_{2}$. Hence both $z_{1}^{\sigma}$ and $z_{2}^{\sigma}$ are multipliers. We take the $\sigma$-th roots of both $z_{1}^{\sigma}$ and $z_{2}^{\sigma}$ to produce multipliers $z_{1}$ and $z_{2}$. We finally form the Jacobian determinant of the two holomorphic function germs $z_{1}$ and $z_{2}$ to conclude that Kohn's algorithm effectively produces the function $F \equiv 1$ as a multiplier. This finishes the proof of the effective termination of Kohn's algorithm in complex dimension 2. 
(III.8) Construction of a New Multiplier in Higher Dimensional Case by Fiberwise Differentiating a Given Multiplier as Many Times as its Multiplicity. We now look at the higher dimensional case. As a preparation for the proof of the Main Theorem for the higher dimensional case, we do the argument here for the construction a new multiplier in higher dimensional case by fiberwise differentiating a given multiplier as many times as its multiplicity. The argument is the same as the 2-dimensional case with corresponding modifications in notations.

We have holomorphic function germs $F_{1}, \cdots, F_{N}$ on $\mathbb{C}^{n}$ at the origin which generate an ideal of multiplicity $q$ whose zero-set is the origin of $\mathbb{C}^{n}$. By applying Corollary (III.4) and (III.5) to get $n \mathbb{C}$-linear combinations of $F_{1}, \cdots, F_{N}$ and form their Jacobian determinant, we get an effectively constructed multiplier $\tilde{h}_{n}\left(z_{1}, \cdots, z_{n}\right)$ at the origin, which vanishes at the origin. The divisor of $\tilde{h}_{n}$ is a subspace germ $V_{n}$ of codimension 1 in $\mathbb{C}^{n}$ at the origin with effectively bounded multiplicity. Because $V_{n}$ has effectively bounded multiplicity, by replacing $\tilde{h}_{n}$ by the product of holomorphic function germs defining the branch germs of the reduction of $V_{n}$, we can assume without loss of generality that $V_{n}$ is a reduced hypersurface germ in $\mathbb{C}^{n}$ at the origin with effectively bounded multiplicity. Again this does not mean that $V_{n}$ is irreducible. It only means that the divisor of $\tilde{h}_{n}$ has coefficient 1 for every one of its irreducible components.

By Proposition (A.3) in Appendix A, the ideal generated by

$$
\frac{\partial\left(F_{i_{1}}, \cdots, F_{i_{n-1}}\right)}{\partial\left(z_{j_{1}}, \cdots, z_{j_{n-1}}\right)}
$$

for $1 \leq i_{1}<\cdots<i_{n-1} \leq N$ and $1 \leq j_{1}<\cdots<j_{n-1} \leq n$ contains an effective power of the maximum ideal $\mathfrak{m}_{\mathbb{C}^{n}, 0}$ of $\mathbb{C}^{n}$ at the origin. Just like the argument given in the 2-dimensional case in (III.7), after a generic $\mathbb{C}$-linear coordinate change and after taking $n-1$ generic $\mathbb{C}$-linear combinations $h_{1}, \cdots, h_{n-1}$ of $F_{1}, \cdots, F_{N}$ we have the following situation.

(i) The ideal generated by $h_{1}, \cdots, h_{n-1}$ and $\tilde{h}_{n}$ has effectively bounded multiplicity at the origin,

(ii) The ideal generated by $\tilde{h}_{n}$ and

$$
\frac{\partial\left(h_{1}, \cdots, h_{n-1}\right)}{\partial\left(z_{1}, \cdots, z_{n-1}\right)}
$$

has effectively bounded multiplicity at the origin. 
(iii) The projection $P \mapsto\left(h_{1}(P), \cdots, h_{n-1}(P)\right)$ makes $V_{n}$ an analytic cover over $\mathbb{C}^{n-1}$ locally at the origin as germs.

(iv) The projection $\left(z_{1}, \cdots, z_{n}\right) \mapsto\left(h_{1}, \cdots, h_{n-1}, z_{n}\right)$ makes $\mathbb{C}^{n}$ an analytic cover over $\mathbb{C}^{n}$ locally at the origin as germs.

Consider the image $\hat{V}_{n}$ of $V_{n}$ under the projection $\left(z_{1}, \cdots, z_{n}\right) \mapsto\left(h_{1}, \cdots, h_{n-1}, z_{n}\right)$ and let

$$
h_{n}=z_{n}^{\lambda}+\sum_{j=0}^{\lambda-1} a_{j}\left(h_{1}, \cdots, h_{n-1}\right) z_{n}^{j}
$$

be the Weierstrass polynomial in the target space $\mathbb{C}^{n}$ with coordinates $\left(h_{1}, \cdots, h_{n-1}, z_{n}\right)$ whose vanishing defines the subspace germ $\hat{V}_{n}$ at the origin in $\mathbb{C}^{n}$. This is possible, because the projection $P \mapsto\left(h_{1}(P), \cdots, h_{n-1}(P)\right)$ makes $V_{n}$ an analytic cover over $\mathbb{C}^{n-1}$ locally at the origin as germs. When regarded as a function-germ in the variables $\left(z_{1}, \cdots, z_{n}\right)$ the function-germ $h_{n}$ contains $\tilde{h}_{n}$ as a factor, because the inverse image of $\hat{V}_{n}$ under the projection $\left(z_{1}, \cdots, z_{n}\right) \mapsto\left(h_{1}, \cdots, h_{n-1}, z_{n}\right)$ contains $V_{n}$ and because $V_{n}$ which is defined by $\tilde{h}_{n}$ is reduced. Since $\tilde{h}_{n}$ is a multiplier, it follows that $h_{n}$ is also a multiplier. Since the ideal generated by $\tilde{h}_{n}$ and

$$
\frac{\partial\left(h_{1}, \cdots, h_{n-1}\right)}{\partial\left(z_{1}, \cdots, z_{n-1}\right)}
$$

has effectively bounded multiplicity at the origin and since $h_{1} \cdots, h_{n-1}$ all vanish at the origin, it follows that there exists some polynomial $p\left(h_{1}, \cdots, h_{n-1}\right)$ such that

(i) the ideal generated by $p\left(h_{1}, \cdots, h_{n-1}\right)$ and $\tilde{h}_{n}$ has effectively bounded multiplicity at the origin, and

(ii) $p\left(h_{1}, \cdots, h_{n-1}\right)$ belongs to the ideal generated by $\tilde{h}_{n}$ and

$$
\frac{\partial\left(h_{1}, \cdots, h_{n-1}\right)}{\partial\left(z_{1}, \cdots, z_{n-1}\right)} .
$$

In particular,

$$
\left|p\left(h_{1}, \cdots, h_{n-1}\right)\right| \lesssim\left|\frac{\partial\left(h_{1}, \cdots, h_{n-1}\right)}{\partial\left(z_{1}, \cdots, z_{n-1}\right)}\right|+\left|\tilde{h}_{n}\right| .
$$

One way to obtain the polynomial $p\left(h_{1}, \cdots, h_{n-1}\right)$ is to use the direct image of the ideal generated by generated by $\tilde{h}_{n}$ and

$$
\frac{\partial\left(h_{1}, \cdots, h_{n-1}\right)}{\partial\left(z_{1}, \cdots, z_{n-1}\right)}
$$


under the local projection $P \mapsto\left(h_{1}(P), \cdots, h_{n-1}(P)\right)$ from $\mathbb{C}^{n}$ to $\mathbb{C}^{n-1}$ and obtain $p\left(h_{1}, \cdots, h_{n-1}\right)$ from the zero-set of this direct image by taking an effective power.

We now form $d h_{1} \wedge \cdots \wedge d h_{n}$ and get

$$
\begin{gathered}
d h_{1} \wedge \cdots \wedge d h_{n}=d h_{1} \wedge \cdots \wedge d h_{n-1} \wedge\left(\lambda z_{n}^{\lambda-1} d z_{n}+\sum_{j=1}^{\lambda-1} j a_{j}\left(h_{1}, \cdots, h_{n-1}\right) z_{n}^{j-1} d z_{n}\right) \\
=\frac{\partial\left(h_{1}, \cdots, h_{n-1}\right)}{\partial\left(z_{1}, \cdots, z_{n-1}\right)}\left(\lambda z_{n}^{\lambda-1}+\sum_{j=1}^{\lambda-1} j a_{j}\left(h_{1}, \cdots, h_{n-1}\right) z_{n}^{j-1}\right) d z_{1} \wedge \cdots \wedge d z_{n} .
\end{gathered}
$$

Since the coefficient of $d z_{1} \wedge \cdots \wedge d z_{n}$ in $d h_{1} \wedge \cdots \wedge d h_{n}$ is a multiplier, it follows that

$$
\frac{\partial\left(h_{1}, \cdots, h_{n-1}\right)}{\partial\left(z_{1}, \cdots, z_{n-1}\right)}\left(\lambda z_{n}^{\lambda-1}+\sum_{j=1}^{\lambda-1} j a_{j}\left(h_{1}, \cdots, h_{n-1}\right) z_{n}^{j-1}\right)
$$

is a multiplier. Since $\tilde{h}_{n}$ is a multiplier, it follows that

$$
\tilde{h}_{n}\left(\lambda z_{n}^{\lambda-1}+\sum_{j=1}^{\lambda-1} j a_{j}\left(h_{1}, \cdots, h_{n-1}\right) z_{n}^{j-1}\right)
$$

is a multiplier. From (III.8.1) it follows that

$$
\begin{gathered}
\left|p\left(h_{1}, \cdots, h_{n-1}\right)\left(\lambda z_{n}^{\lambda-1}+\sum_{j=1}^{\lambda-1} j a_{j}\left(h_{1}, \cdots, h_{n-1}\right) z_{n}^{j-1}\right)\right| \\
\lesssim\left|\frac{\partial\left(h_{1}, \cdots, h_{n-1}\right)}{\partial\left(z_{1}, \cdots, z_{n-1}\right)}\left(\lambda z_{n}^{\lambda-1}+\sum_{j=1}^{\lambda-1} j a_{j}\left(h_{1}, \cdots, h_{n-1}\right) z_{n}^{j-1}\right)\right| \\
+\left|\tilde{h}_{n}\left(\lambda z_{n}^{\lambda-1}+\sum_{j=1}^{\lambda-1} j a_{j}\left(h_{1}, \cdots, h_{n-1}\right) z_{n}^{j-1}\right)\right| .
\end{gathered}
$$

Hence by the Real Radical Property of Kohn's algorithm in (I.1)(C),

$$
p\left(h_{1}, \cdots, h_{n-1}\right)\left(\lambda z_{n}^{\lambda-1}+\sum_{j=1}^{\lambda-1} j a_{j}\left(h_{1}, \cdots, h_{n-1}\right) z_{n}^{j-1}\right)
$$


is a multiplier. Let $h_{n}^{(0)}=h_{n}$ and for $1 \leq \nu \leq \lambda$ define

$$
h_{n}^{(\nu)}=p\left(h_{1}, \cdots, h_{n-1}\right)^{\nu}\left(\frac{\lambda !}{(\lambda-\nu) !} z_{n}^{\lambda-\nu}+\sum_{j=\nu}^{\lambda-1} \frac{j !}{(j-\nu) !} a_{j}\left(h_{1}, \cdots, h_{n-1}\right) z_{n}^{j-\nu}\right) .
$$

We are going to verify by induction on $\nu$ that $h_{n}^{(\nu)}$ is a multiplier. We know that both $h_{n}^{(0)}$ and $h_{n}^{(1)}$ are multipliers. Assume that we have already verified that $h_{n}^{(0)}, \cdots, h_{n}^{(\nu-1)}$ are multipliers. Then $d h_{1} \wedge \cdots \wedge d h_{n-1} \wedge d h_{n}^{(\nu-1)}$ is equal to

$$
\begin{array}{r}
d h_{1} \wedge \cdots \wedge d h_{n-1} \wedge d\left(p ( h _ { 1 } , \cdots , h _ { n - 1 } ) ^ { \nu - 1 } \left(\frac{\lambda !}{(\lambda-\nu+1) !} z_{n}^{\lambda-\nu+1}\right.\right. \\
\left.\left.\quad+\sum_{j=\nu-1}^{\lambda-1} \frac{j !}{(j-\nu+1) !} a_{j}\left(h_{1}, \cdots, h_{n-1}\right) z_{n}^{j-\nu+1}\right)\right) \\
=d h_{1} \wedge \cdots \wedge d h_{n-1} \wedge\left(p ( h _ { 1 } , \cdots , h _ { n - 1 } ) ^ { \nu - 1 } \left(\frac{\lambda !}{(\lambda-\nu) !} z_{n}^{\lambda-\nu}\right.\right. \\
\left.\left.+\sum_{j=\nu}^{\lambda-1} \frac{j !}{(j-\nu) !} a_{j}\left(h_{1}, \cdots, h_{n-1}\right) z_{n}^{j-\nu}\right) d z_{n}\right) \\
=\frac{\partial\left(h_{1}, \cdots, h_{n-1}\right)}{\partial\left(z_{1}, \cdots, z_{n-1}\right)}\left(p\left(h_{1}, \cdots, h_{n-1}\right)^{\nu-1}\left(\frac{\lambda !}{(\lambda-\nu) !} z_{n}^{\lambda-\nu}\right)\right. \\
\left.\left.+\sum_{j=\nu}^{\lambda-1} \frac{j !}{(j-\nu) !} a_{j}\left(h_{1}, \cdots, h_{n-1}\right) z_{n}^{j-\nu}\right)\right) d z_{1} \wedge \cdots \wedge d z_{n} .
\end{array}
$$

Since the coefficient of $d z_{1} \wedge \cdots \wedge d z_{n}$ in $d h_{1} \wedge \cdots \wedge d h_{n-1} \wedge d h_{n}^{(\nu-1)}$ is a multiplier, it follows that

$$
\begin{aligned}
\frac{\partial\left(h_{1}, \cdots, h_{n-1}\right)}{\partial\left(z_{1}, \cdots, z_{n-1}\right)}\left(p \left(h_{1}, \cdots,\right.\right. & \left., h_{n-1}\right)^{\nu-1}\left(\frac{\lambda !}{(\lambda-\nu) !} z_{n}^{\lambda-\nu}\right. \\
& \left.\left.+\sum_{j=\nu}^{\lambda-1} \frac{j !}{(j-\nu) !} a_{j}\left(h_{1}, \cdots, h_{n-1}\right) z_{n}^{j-\nu}\right)\right)
\end{aligned}
$$

is a multiplier. Since $\tilde{h}_{n}$ is a multiplier, it follows that

$$
\tilde{h}_{n}\left(p\left(h_{1}, \cdots, h_{n-1}\right)^{\nu-1}\left(\frac{\lambda !}{(\lambda-\nu) !} z_{n}^{\lambda-\nu}+\sum_{j=\nu}^{\lambda-1} \frac{j !}{(j-\nu) !} a_{j}\left(h_{1}, \cdots, h_{n-1}\right) z_{n}^{j-\nu}\right)\right)
$$


is a multiplier. From (III.8.1) it follows that

$$
\begin{array}{r}
\mid p\left(h_{1}, \cdots, h_{n-1}\right)\left(p ( h _ { 1 } , \cdots , h _ { n - 1 } ) ^ { \nu - 1 } \left(\frac{\lambda !}{(\lambda-\nu) !} z_{n}^{\lambda-\nu}\right.\right. \\
\left.\left.+\sum_{j=\nu}^{\lambda-1} \frac{j !}{(j-\nu) !} a_{j}\left(h_{1}, \cdots, h_{n-1}\right) z_{n}^{j-\nu}\right)\right) \mid \\
\lesssim \mid \frac{\partial\left(h_{1}, \cdots, h_{n-1}\right)}{\partial\left(z_{1}, \cdots, z_{n-1}\right)}\left(p ( h _ { 1 } , \cdots , h _ { n - 1 } ) ^ { \nu - 1 } \left(\frac{\lambda !}{(\lambda-\nu) !} z_{n}^{\lambda-\nu}\right.\right. \\
\left.\left.+\sum_{j=\nu}^{\lambda-1} \frac{j !}{(j-\nu) !} a_{j}\left(h_{1}, \cdots, h_{n-1}\right) z_{n}^{j-\nu}\right)\right) \mid \\
+\mid \tilde{h}_{n}\left(p\left(h_{1}, \cdots, h_{n-1}\right)^{\nu-1}\left(\frac{\lambda !}{(\lambda-\nu) !} z_{n}^{\lambda-\nu}\right)\right. \\
\left.\left.+\sum_{j=\nu}^{\lambda-1} \frac{j !}{(j-\nu) !} a_{j}\left(h_{1}, \cdots, h_{n-1}\right) z_{n}^{j-\nu}\right)\right) \mid
\end{array}
$$

Hence by the Real Radical Property of Kohn's algorithm in (I.1)(C),

$$
\begin{aligned}
p\left(h_{1}, \cdots, h_{n-1}\right)\left(p \left(h_{1}, \cdots,\right.\right. & \left.h_{n-1}\right)^{\nu-1}\left(\frac{\lambda !}{(\lambda-\nu) !} z_{n}^{\lambda-\nu}\right. \\
& \left.\left.+\sum_{j=\nu}^{\lambda-1} \frac{j !}{(j-\nu) !} a_{j}\left(h_{1}, \cdots, h_{n-1}\right) z_{n}^{j-\nu}\right)\right)
\end{aligned}
$$

is a multiplier and $h_{n}^{(\nu)}$ is a multiplier. When $\nu=\lambda$, we end up with

$$
h_{n}^{(\lambda)}=p\left(h_{1}, \cdots, h_{n-1}\right)^{\lambda} \lambda !
$$

being a multiplier. Since the multiplicity of the ideal generated by $p\left(h_{1}, \cdots, h_{n-1}\right)$ and $\tilde{h}_{n}$ is effectively bounded at the origin, it follows that the multiplicity of the ideal generated by $p\left(h_{1}, \cdots, h_{n-1}\right)^{\lambda}$ and $\tilde{h}_{n}$ is effectively bounded at the origin. We can conclude that $p\left(h_{1}, \cdots, h_{n-1}\right)$ is a multiplier admitting an order of subellipticity with an effective positive lower bound.

(III.9) Effective Termination of Kohn's Algorithm in the Higher Dimensional Case. Recall that in (III.8) we have the multiplier $h_{n}$ constructed from $F_{1}, \cdots, F_{N}$ and $\tilde{h}_{n}$ by choosing $n-1$ good $\mathbb{C}$-linear combinations of $F_{1}, \cdots, F_{N}$. Now we enhance the construction of $h_{n}$ by choosing $n$ good $\mathbb{C}$-linear combinations of $F_{1}, \cdots, F_{N}$ so that any subset of $n-1$ of them are good $\mathbb{C}$-linear combinations 
for our purpose. More precisely, as in (III.8) we choose $n$ generic $\mathbb{C}$-linear combinations $H_{1}, \cdots, H_{n}$ of $F_{1}, \cdots, F_{N}$ such that

(i) the map $\pi: \mathbb{C}^{n} \rightarrow \mathbb{C}^{n}$ defined by $H_{1}, \cdots, H_{n}$ is an analytic cover map locally at the origin whose number of sheets is effectively bounded, and

(ii) for any $1 \leq j \leq n$ we can use $H_{1}, \cdots, H_{j-1}, H_{j+1}, \cdots, H_{n}$ as $h_{1}, \cdots, h_{n-1}$ for the argument in (III.8) to produce a polynomial $p_{j}\left(H_{1}, \cdots, H_{j-1}, H_{j+1}, \cdots, H_{n}\right)$ of $H_{1}, \cdots, H_{j-1}, H_{j+1}, \cdots, H_{n}$ which is a multiplier and whose multiplicity at the origin is effectively bounded and whose assigned order of subellipticity has an effective positive lower bound.

The argument in (III.8) shows that each $p_{j}\left(H_{1}, \cdots, H_{j-1}, H_{j+1}, \cdots, H_{n}\right)$ is an effectively constructed multiplier for $1 \leq j \leq n$.

We introduce coordinates $z_{1}, \cdots, z_{n}$ in the domain space $\mathbb{C}^{n}$ of the map $\pi$ : $\mathbb{C}^{n} \rightarrow \mathbb{C}^{n}$. We use coordinates $w_{1}, \cdots, w_{n}$ in the target space $\mathbb{C}^{n}$ of the map $\pi: \mathbb{C}^{n} \rightarrow \mathbb{C}^{n}$. Since the polynomial $p_{j}\left(w_{1}, \cdots, w_{j-1}, w_{j+1}, \cdots, w_{n}\right)$ as a function of $w_{1}, \cdots, w_{j-1}, w_{j+1}, \cdots, w_{n}$ has effectively bounded multiplicity at the origin. it follows that an effectively bounded positive power of the maximum ideal of the target space $\mathbb{C}^{n}$ of $\pi$ at the origin is contained in the ideal of the target space $\mathbb{C}^{n}$ of $\pi$ at the origin generated by the $n$ polynomials $p_{j}\left(w_{1}, \cdots, w_{j-1}, w_{j+1}, \cdots, w_{n}\right)$ for $1 \leq j \leq n$.

Since the map $\pi: \mathbb{C}^{n} \rightarrow \mathbb{C}^{n}$ defined by $H_{1}, \cdots, H_{n}$ is an analytic cover map locally at the origin whose number of sheets is effectively bounded, it follows that an an effectively bounded positive power of the maximum ideal of the domain space $\mathbb{C}^{n}$ of $\pi$ at the origin is contained in the ideal of the domain space $\mathbb{C}^{n}$ of $\pi$ at the origin generated by the $n$ holomorphic function germs $p_{j}\left(H_{1}, \cdots, H_{j-1}, H_{j+1}, \cdots, H_{n}\right)$ for $1 \leq j \leq n$. Since each $p_{j}\left(H_{1}, \cdots, H_{j-1}, H_{j+1}, \cdots, H_{n}\right)$ is an effectively constructed multiplier for $1 \leq$ $j \leq n$, it follows that each of the coordinates $z_{1}, \cdots, z_{n}$ of the domain space $\mathbb{C}^{n}$ of $\pi$ is a multiplier with effective assigned order of subellipticity. By forming the Jacobian determinant of the multipliers $z_{1}, \cdots, z_{n}$, we conclude that the function $F \equiv 1$ is a multiplier whose assigned order of subellipticity has an effective positive lower bound. This finishes the proof of the effective termination of Kohn's algorithm and concludes the proof of Main Theorem (III.2). 
(III.10) Remark on the Need to Fiberwise Differentiate as Many Times as the Multiplicity of the Given Multiplier. An earlier version of this paper puts in the proof only one fiberwise differentiation for the given multiplier instead of the number of fiber differentiations equal to the multiplicity of the multiplier. This version adds the required number of differentiations. Let us explain the need to fiberwise differentiate as many times as the multiplicity of the multiplier by considering the following simple situation in complex dimension 2.

Let $f(z, w)$ be a Weierstrass polynomial of degree $q$ in $w$, which is a monic polynomial in $w$ whose coefficients, except the leading one, are holomorphic function germs in $z$ vanishing at the origin. Denote by $f_{w}(z, w)$ the derivative of $f(z, w)$ with respect to $w$. Let $D(z)$ be the discriminant of $f(z, w)$ as a polynomial in $w$. Then Euclid's algorithm gives $D(z)=a(z, w) f(z, w)+b(z, w) f_{w}(z, w)$, where $a(z, w)$ and $b(z, w)$ are holomorphic function germs on $\mathbb{C}^{2}$ at the origin.

Note that if $q$ is small, we can only conclude that the multiplicity $q$ of $f(z, w)$ at the origin is small and we cannot conclude that the coefficients of powers of $w$, other than the leading one, have low vanishing order in $z$ at $z=0$.

Suppose $f(z, w)$ is a multiplier and $z$ is a pre-multiplier. When we apply the operator $d z \wedge d(\cdot)$ to $f(z, w)$ to get $f_{w}(z, w) d z \wedge d w$, we conclude that $f_{w}(z, w)$ is a multiplier. From $D(z)=a(z, w) f(z, w)+b(z, w) f_{w}(z, w)$ it follows that the discriminant $D(z)$ is a also multiplier which in general is not effectively constructed. The vanishing order of $D(z)$ in $z$ at $z=0$ in general does not have anything to do with $q$ and certainly in general cannot be bounded by an effective function of $q$. Thus the ideal generated by the multipliers $f(z, w)$ and $D(z)$ may have high multiplicity at the origin if $D(z)$ has high vanishing order in $z$ at $z=0$. This function germ $D(z)$ is obtained by one single fiberwise differentiation of the multiplier $f(z, w)$.

The discriminant $D(z)$ is given by $\prod_{i \neq j}\left(w_{i}(z)-w_{j}(z)\right)^{2}$, where $\left\{w_{1}(z), \cdots, w_{q}(z)\right\}$ (without any well-defined ordering) is the collection of the $q$ roots of $f(z, w)$ in $w$ with the multiplicities of the roots counted. If the minimum distance of two points in $\left\{w_{1}(z), \cdots, w_{q}(z)\right\}$ as a function of $z$ vanishes to high order in $z$ at $z=0$, we would have high vanishing order for $D(z)$. The process of getting $D(z)$ by differentiating once does not help in our goal of achieving an effective termination of Kohn's algorithm when two of the roots from the set $\left\{w_{1}(z), \cdots, w_{q}(z)\right\}$ 
are becoming close very fast as $z$ approaches 0 . Since we have no control over how fast some of the roots $\left\{w_{1}(z), \cdots, w_{q}(z)\right\}$ are getting close as $z \rightarrow 0$, we need to differentiate $q$ times in order to achieve our goal of an effective termination of Kohn's algorithm. This explains why we need to fiberwise differentiate as many times as the multiplicity of the multiplier.

(III.11) Motivation of the Proof of Termination of Kohn's Algorithm from the Fundamental Theorem in Multivariate Calculus for Fubini's Iterated Integration. We would like to remark that the motivation for the above proof of the termination of Kohn's algorithm for special domains comes from the fundamental theorem in multivariate calculus for the following theorem of Fubini on iterated integration. The reason for this motivation is that Jacobian determinants occur in the change-of-variables formula for integrals of several variables and that an induction process can be used when we convert an integral of several variables to an iterated integral by Fubini's theorem.

(III.11.1) Fubini's Iterated Integration. Let $y_{1}, \cdots, y_{n-1}$ be functions defining a projection from an $n$-space $G$ with coordinates $x_{1}, \cdots, x_{n}$ to an $(n-1)$-space $D$ with coordinates $y=\left(y_{1}, \cdots, y_{n-1}\right)$ so that $x_{n}$ can be used to be a local coordinate for the fiber $L_{y}$ of the projection over the point $y \in D$. Then for a function $f$ on $G$ the formula

$$
\int_{G} f=\int_{y \in D}\left(\int_{L_{y}} f\right)
$$

holds with the use of appropriate measures.

Like the fundamental theorem of calculus of a single real variable, the fundamental theorem in multivariate calculus for the above theorem of Fubini on iterated integration changes integration to differentiation. If we write the function $f$ in the form $f\left(y_{1}, \cdots, y_{n-1}, x_{n}\right)$, then

$$
d y_{1} \wedge \cdots \wedge d y_{n-1} \wedge d f=d y_{1} \wedge \cdots \wedge d y_{n-1} \wedge\left(\frac{\partial f}{\partial x_{n}}\right) d x_{n}
$$

so that fiberwise integration over $L_{y}$ with respect to $x_{n}$ in (III.11.1) changes over to fiberwise differentiation on $L_{y}$ with respect to $x_{n}$. 
When we use a multiplier as $f$ and pre-multipliers as $y_{1}, \cdots, y_{n-1}$ to form the Jacobian determinant with respect to $x_{1}, \cdots, x_{n}$, we get

$$
\begin{gathered}
d y_{1} \wedge \cdots \wedge d y_{n-1} \wedge d f \\
=\frac{\partial\left(y_{1}, \cdots, y_{n-1}\right)}{\partial\left(x_{1}, \cdots, x_{n-1}\right)}\left(\frac{\partial f}{\partial x_{n}}\right)\left(d x_{1} \wedge \cdots \wedge d x_{n}\right) .
\end{gathered}
$$

The occurrence of the factor $\frac{\partial f}{\partial x_{n}}$ enables us to reduce the vanishing order of $f$ by differentiation and the occurrence, as a factor, of the Jacobian determinant

$$
\frac{\partial\left(y_{1}, \cdots, y_{n-1}\right)}{\partial\left(x_{1}, \cdots, x_{n-1}\right)}
$$

involving one fewer variable makes it possible to use an induction process.

\section{Part IV. Geometric Formulation of Kohn's Algorithm in Terms of Frobenius Theorem on Integral Submanifolds and the Rôle of Real- Analyticity}

Kohn's conjecture for the real-analytic case without effectiveness was proved by Diederich-Fornaess [DF78]. We are going to formulate Kohn's algorithm geometrically in terms of the theorem of Frobenius on integral submanifolds and present a proof of the real-analytic case of the ineffective termination of Kohn's algorithm from the geometric viewpoint. This geometric formulation of Kohn's algorithm in terms of the theorem of Frobenius enables one to see clearly how the procedures of Kohn's algorithm come about naturally in the geometric context. Moreover, the proof of the real-analytic case of the ineffective termination of Kohn's algorithm from the geometric viewpoint gives a better understanding of the rôle played by the real-analytic assumption and of the hurdles standing in the way of generalizing the ineffective real-analytic case to the ineffective smooth case.

(IV.1) Usual Theorem of Frobenius on Integral Submanifolds for $\mathbb{R}^{m}$. The setting of the usual Frobenius theorem on integral submanifolds of real dimension $k$ starts out with a domain $U$ in $\mathbb{R}^{m}$ and a distribution

$$
x \mapsto W_{x} \subset T_{\mathbb{R}^{m}, x}=\mathbb{R}^{m} \text { for } x \in U
$$

which is smooth, where $W_{x}$ is a $k$-dimensional $\mathbb{R}$-linear subspace of the tangent space $T_{\mathbb{R}^{n}, x}$ of $\mathbb{R}^{n}$ at $x$. 
The vector-field version of Frobenius's theorem states that the distribution $x \mapsto W_{x}$ is locally integrable (in the sense that locally $U$ is foliated by smooth real submanifolds of real dimension $k$ whose tangent space at the point $x$ is precisely $W_{x}$ ) if and only if $\left[W_{x}, W_{x}\right] \subset W_{x}$ for all $x \in U$ (in the sense that for all $x \in U$ the value at $x$ of the Lie bracket of two local vector fields whose values at $y$ in their domains of definition are in $W_{y}$ for each $y$ belongs to $W_{x}$ ).

The equivalent differential-form version of Frobenius's theorem states that the distribution $x \mapsto W_{x}$ is locally integrable if and only if for any local smooth differential 1-form $\omega_{1}, \cdots, \omega_{m-k}$ whose common kernel is $W_{x}$ there exist local smooth differential 1-forms $\eta_{1}, \cdots, \eta_{m-k}$ such that $d \omega_{j}=\sum_{\ell=1}^{m-k} \omega_{\ell} \wedge \eta_{\ell}$ for $1 \leq$ $j \leq m-k$.

The vector-field version of Frobenius's theorem is related to its differential-form version by Cartan's formula relating Lie brackets of vector fields and the exterior differentiation of differential forms (see, for example, [He62, p.21, Formula (9)]).

(IV.2) Setting of CR Hypersurface for the Theorem of Frobenius Theorem. In the formulation of Kohn's algorithm in terms of Frobenius's theorem the setting is the boundary $S$ of a bounded smooth domain $\Omega$ in $\mathbb{C}^{n}$ and the distribution on $S$ is $P \mapsto T_{S, P}^{\mathbb{R}} \cap J\left(T_{S, P}^{\mathbb{R}}\right)$, where $T_{S, P}^{\mathbb{R}}$ is the space of all real tangent vectors in $S$ at $P$ and $J$ is the almost-complex operator of $\mathbb{C}^{n}$.

In this setting the condition of the theorem of Frobenius is equivalent to $S$ being Levi-flat, in which case $S$ is locally foliated by local complex submanifolds of complex dimension $n-1$.

The finite type condition of d'Angelo is the opposite of being Levi-flat. The finite type condition of d'Angelo can be interpreted in this context as the impossibility of finding even Artinian subschemes of arbitrarily high order in $S$ which are tangential to the distribution $x \mapsto W_{x}$ of $S$. The underlying point set of an Artinian subscheme is just a single point, but its structure sheaf may be more than the complex number field $\mathbb{C}$ and can be an Artinian ring (i.e. a ring of finite dimension) which is the quotient of the structure sheaf of $S$.

We will not go into the precise definition an Artinian subscheme here. Its definition depends on the structure sheaf of $S$ which in the real-analytic case is the sheaf of germs of all real-analytic functions and in the smooth case is the sheaf 
of germs of all smooth functions. As an illustration we give here the following two examples of Artinian subschemes $\mathcal{A}$ of the ringed space $\left(\mathbb{C}^{n}, \mathcal{O}_{\mathbb{C}^{n}}\right)$ supported at the origin of $\mathbb{C}^{n}$, where $\mathcal{O}_{\mathbb{C}^{n}}$ is the sheaf of all holomorphic function germs on $\mathbb{C}^{n}$.

Let $\mathfrak{m}_{\mathbb{C}^{n}, 0}$ be the maximum ideal at the origin of $\mathbb{C}^{n}$. Then the ringed space $\left(\{0\}, \mathcal{O}_{\mathbb{C}^{n}} /\left(\mathfrak{m}_{\mathbb{C}^{n}, 0}\right)^{q}\right)$ for any positive integer $q$ is an Artinian subscheme of the ringed space $\left(\mathbb{C}^{n}, \mathcal{O}_{\mathbb{C}^{n}}\right)$ supported at the origin of $\mathbb{C}^{n}$. For any ideal $\mathcal{I}$ of $\mathcal{O}_{\mathbb{C}^{n}, 0}$ with $\left(\mathfrak{m}_{\mathbb{C}^{n}, 0}\right)^{N} \subset \mathcal{I}$ for some positive integer $N$, the ringed space $\left(\{0\}, \mathcal{O}_{\mathbb{C}^{n}} / \mathcal{I}\right)$ is also an Artinian subscheme of the ringed space $\left(\mathbb{C}^{n}, \mathcal{O}_{\mathbb{C}^{n}}\right)$ supported at the origin of $\mathbb{C}^{n}$.

(IV.3) Steps of Kohn's Algorithm from Constructing Integral Complex Curves. We are going to see how the steps of Kohn's algorithm naturally arise in the study of conditions necessary for the construction of integral complex curves in the boundary of a smooth bounded domain. Again the setting is a weakly pseudoconvex smooth bounded domain $\Omega$ with boundary $S$ and again on $S$ we consider the distribution $P \mapsto T_{S, P}^{\mathbb{R}} \cap J\left(T_{S, P}^{\mathbb{R}}\right)$ for $P \in S$. We will later specialize to the case where the boundary $S$ of the bounded domain $\Omega$ is real-analytic and will investigate precisely the rôle played by the assumption of real-analyticity of $S$. To anticipate the later specialization into the case of the boundary $S$ being real-analytic, we would like to explore conditions which give as a consequence the existence of some local complex curve in $S$. What we would like to do is to assume that Kohn's algorithm does not terminate and seek to produce geometrically a local complex curve in $S$ in the real-analytic case. For this purpose, in our discussion, from time to time we will restrict ourselves to some appropriate open subsets of $S$ in order to exclude the singularity of real-analytic subsets which arise in our discussion.

Let $N_{S}^{(1,0)}$ be the set of all $(1,0)$-vectors of $S$ which is in the null space of the Levi form of $S$. Let $\mathcal{N}$ be the real part of $N_{S}^{(1,0)}$ in the sense that at a point $P$ of $S$ the space $\mathcal{N}$ consists of all $\operatorname{Re} \xi$ with $\xi \in N_{S}^{(1,0)}$ at the point $P$. Let $T_{S}^{\mathbb{R}}$ be the vector bundle of all real tangent vectors of $S$. One key property of $\mathcal{N}$ is the following.

(IV.3.1) Let $P$ be a point of $S$ and $U$ be an open neighborhood of $P$ in $S$. Let $\xi$ and $\eta$ be smooth sections of $T_{S}^{\mathbb{R}}$ over $U$. That is, $\xi$ and $\eta$ are real tangent vector 
fields of $S$ defined on $U$. Assume that both $\xi$ and $\eta$ belongs to $\mathcal{N}$ at $P$. Then the value of the Lie bracket $[\xi, \eta]$ at $P$ belongs to $\mathcal{N}$.

Another way to state (IV.3.1) is the following.

(IV.3.2) The real part of the null space of $(1,0)$-vectors for the Levi form of a weakly pseudoconvex boundary is closed under the Lie bracket after extension of the pointwise vectors to vector fields.

The statement (IV.3.1) holds mainly because on $S$ the first derivative of the Levi form for a $(1,0)$-vector field vanishes at any of its zero points. The details for its proof are given in [DF78, Proposition 1].

Let $G$ be a nonempty open subset of $S$ where the real dimension of $\mathcal{N}$ is constant, say $\ell$. For the case $\ell \geq 1$, it follows from Frobenius theorem and condition (IV.3.1) that over $G$ we can integrate $\mathcal{N}$ to get local integral submanifolds $M$ of $G$ of real dimension $\ell$ so that the tangent space of $M$ at any point $P$ of $M$ is equal to the real vector space $\mathcal{N}$ at $P$.

Since at every point $P$ of $S$ the space $N_{S}^{(1,0)}$ is a vector space over the complex number field $\mathbb{C}$, we know that its real part $\mathcal{N}$ must be invariant under the almost-complex-structure operator $J$. Thus we can conclude that the tangent space $\mathcal{N}$ of each local integral submanifold $M$ is invariant under the almostcomplex-structure operator $J$. This implies that each $M$ is complex-analytic. As a consequence, one has the following trivial remark.

(IV.4) Remark. Suppose $S$ is a local smooth weakly pseudoconvex hypersurface in $\mathbb{C}^{n}$. If at each point of $S$ the null space of the Levi form of $S$ is nontrivial, then some nonempty open subset $G$ of $S$ is foliated by local complex submanifolds of positive dimension.

(IV.5) Natural Occurrence of the Steps of Kohn's Algorithm. The algorithm of Kohn comes into the picture only when we do not have a nonempty open subset $G$ of $S$ where the real dimension of $\mathcal{N}$ is some positive constant $\ell$. We consider the set $E$ of points of $S$ where the real dimension of $\mathcal{N}$ is some positive constant $\ell$. The case of interest is when $E$ does not contain an open subset of $S$. This step of introducing $E$ corresponds to introducing the coefficients of the $(n, n-1)$-form $\partial r \wedge(\partial \bar{\partial} r)^{n-1}$ as multipliers in Kohn's algorithm. We are going to assume that $E$ is a smooth submanifold of real dimension $m \geq 1$ and that $\left.\mathcal{N}\right|_{E}$ is a smooth 
vector bundle over $E$. In the real-analytic case because of the stratification of real-analytic subvarieties we can always get to a real-analytic submanifold and a real-analytic bundle by replacing the point under consideration by another point nearby. In the general smooth case there is no such stratification and the situation becomes complicated and calls for other techniques than those discussed here.

We want to apply Frobenius's theorem to $E$ with the distribution of vector spaces $\mathcal{N}$ over it. The trouble is that the vector space $\mathcal{N}$ at a point $P$ of $E$ may not be inside the real tangent space $T_{E, P}^{\mathbb{R}}=\left(T_{E}^{\mathbb{R}}\right)_{P}$ of $E$ at $P$. To apply Frobenius's theorem to $E$ we must work with a distribution of subspaces of the tangent spaces of $E$. We are forced to replace $\mathcal{N}$ by $\mathcal{N} \cap T_{E}^{\mathbb{R}}$ at each point $P$ of $E$. We also want to keep the smaller new vector space $\mathcal{N} \cap T_{E}^{\mathbb{R}}$ invariant under the almost-complex-structure operator $J$, because we are interested in producing local complex curves inside $S$. We use the even smaller vector space $\mathcal{N} \cap T_{E}^{\mathbb{R}} \cap J T_{E}^{\mathbb{R}}$. (Note that $\mathcal{N}$ is invariant under $J$.) Then we consider the new subset $E_{1}$ of $E$ where the real dimension of $\mathcal{N} \cap T_{E}^{\mathbb{R}} \cap J T_{E}^{\mathbb{R}}$ is positive.

For the real-analytic case this step corresponds to introducing real-valued realanalytic function germs $f$ vanishing on $E$ as multipliers and also $\partial f$ as vectormultipliers. The reason is that taking intersection with $T_{E}^{\mathbb{R}}$ is the same as considering the kernel of the differential $d f$ of local real-valued functions $f$ on $S$ which vanish identically in $E$. Taking the further intersection with $J T_{E}^{\mathbb{R}}$ is to consider also the kernel of the $J$-image $J d f$ of the differential $d f$ of local real-valued functions $f$ on $S$ which vanish on $E$. Taking both intersections together is the same as considering the kernel of $\partial f$ for local real-valued functions $f$ on $S$ which vanish on $E$. Th use of all local real-analytic function germs vanishing on $E$ tells us how the step (I.1)(C) of Kohn's algorithm naturally arises from the geometric viewpoint. The use of $\partial f$ tells us how the step (I.1)(B)(i) of Kohn's algorithm naturally arises from the geometric viewpoint.

As the initial steps of an inductive process we set $E_{0}=E$ and $\mathcal{N}^{(0)}=\mathcal{N}$ and $\mathcal{N}^{(1)}=\mathcal{N}^{(0)} \cap T_{E_{0}}^{\mathbb{R}} \cap J T_{E_{0}}^{\mathbb{R}}$. Then we inductively define

$$
\mathcal{N}^{(\nu+1)}=\mathcal{N}^{(\nu)} \cap T_{E_{\nu}}^{\mathbb{R}} \cap J T_{E_{\nu}}^{\mathbb{R}}=\mathcal{N} \cap T_{E_{\nu}}^{\mathbb{R}} \cap J T_{E_{\nu}}^{\mathbb{R}}
$$

and define $E_{\nu+1}$ to be the subset of $E$ where the real dimension of $\mathcal{N}^{(\nu)}$ is positive. We obtain the limiting common intersection $E_{\infty}$ defined by $E_{\infty}=\cap_{\nu} E_{\nu}$. By replacing $E_{\infty}$ by a nonempty open subset in the real-analytic case, we can assume 
that $E_{\infty}$ is regular and $\mathcal{N}^{(\infty)}:=\mathcal{N} \cap T_{E_{\infty}}^{\mathbb{R}} \cap J T_{E_{\infty}}^{\mathbb{R}}$ is a real-analytic vector bundle over $E_{\infty}$. Note that, though we go to the regular part of $E_{\nu}$ in order to describe more easily the tangent bundle $T_{E_{\nu}}$ of $E_{\nu}$, when we take the intersection $E_{\infty}=\cap_{\nu} E_{\nu}$ we have to make sure that the intersection $E_{\infty}=\cap_{\nu} E_{\nu}$ is defined in the real-analytic case as a real-analytic subvariety, which forces us to consider $T_{E_{\nu}}$ also at singular points of $E_{\nu}$ where it is defined as the common kernel of differentials of all local real-analytic functions vanishing on $E_{\nu}$.

Note that the definition of $E_{\nu+1}$ as the subset of $E$ where the real dimension of $\mathcal{N}^{(\nu)}$ is positive involves the existence of a nontrivial solution in a system of homogeneous linear equations or equivalently the vanishing of the determinant of the coefficient matrix or equivalently the vanishing of the corresponding exterior product of co-vectors. This tells us how the step of Kohn's algorithm described in (I.1)(B)(ii) naturally arises from the geometric viewpoint.

Now the distribution of vector spaces $\mathcal{N}^{(\infty)}=\mathcal{N} \cap T_{E_{\infty}}^{\mathbb{R}} \cap J T_{E_{\infty}}^{\mathbb{R}}$ is contained in the tangent space of $E_{\infty}$ and each $\mathcal{N}^{(\infty)}=\mathcal{N} \cap T_{E_{\infty}}^{\mathbb{R}} \cap J T_{E_{\infty}}^{\mathbb{R}}$ is $J$-invariant. For the purpose of understanding how the procedures of Kohn's algorithm come about naturally in the geometric context, we assume that each fiber of $\mathcal{N}^{(\infty)}=\mathcal{N} \cap$ $T_{E_{\infty}}^{\mathbb{R}} \cap J T_{E_{\infty}}^{\mathbb{R}}$ is of positive dimension and we also assume that we are in the realanalytic case so that we have the benefit of stratification. Under such assumptions and after restriction to a dense open subset if necessary, $E_{\infty}$ is a CR manifold and has holomorphic dimension at least 1 . However, for $\mathcal{N}^{(\infty)}=\mathcal{N} \cap T_{E_{\infty}}^{\mathbb{R}} \cap J T_{E_{\infty}}^{\mathbb{R}}$ we may not have the involutive condition of the theorem of Frobenius (which means closure under Lie bracket). In order to apply the theorem of Frobenius we generate a larger linear subspace of the tangent space of $E_{\infty}$ by taking iterated Lie brackets of local sections of the vector bundle $\mathcal{N}^{(\infty)}=\mathcal{N} \cap T_{E_{\infty}}^{\mathbb{R}} \cap J T_{E_{\infty}}^{\mathbb{R}}$ to generate a new distribution $\tilde{\mathcal{N}}$. This new distribution $\tilde{\mathcal{N}}$ now satisfies the following three conditions.

(i) $\tilde{\mathcal{N}}$ is contained in the tangent space of $E_{\infty}$.

(ii) $\tilde{\mathcal{N}}$ is involutive in the sense that it is closed under Lie bracket.

(iii) $\tilde{\mathcal{N}}$ belongs to the real part of the null space of the Levi form of $S$.

Note that Condition (iii) is a consequence of (IV.3.1). However, in general $\tilde{\mathcal{N}}$ is no longer $J$-invariant. An integral submanifold $M$ of $\tilde{\mathcal{N}}$ has the following property. At each point of $M$ the holomorphic dimension of $S$ is at least 1 . An open dense 
subset of $M$ is a CR manifold, but in general $M$ is not complex-analytic. We are going to show, with our present assumption of real-analyticity, that when the Kohn algorithm does not terminate, we are able to produce some local complex curve inside $M$. One key point here is that the tangent space of $M$ is contained in the null space $\mathcal{N}$ of the Levi-form which is $J$-invariant.

Since we have assumed that we are in the real-analytic case, at a generic point of $M$ we can consider the smallest complex submanifold germ $V$ in $\mathbb{C}^{n}$ which contains the germ of $M$ at that point. We then have the following situation. At a generic point $P_{0}$ of $M$ there exist

(i) an open neighborhood $U_{0}$ of $P_{0}$ in $\mathbb{C}^{n}$,

(ii) a complex submanifold $V$ in $U_{0}$, and

(iii) real-valued real-analytic functions $\rho_{1}, \cdots, \rho_{\ell}$ on $V$

such that

(a) $M \cap U_{0}$ is the common zero-set of $\rho_{1}, \cdots, \rho_{\ell}$,

(b) $\partial \rho_{1}, \cdots, \partial \rho_{\ell}$ are $\mathbb{C}$-linearly independent at points of $M \cap U_{0}$, and

(c) at any point of $M \cap U_{0}$ the tangent space of $V$ is contained in $\mathcal{N}$.

Condition (b) means that, besides the $\mathbb{R}$-linear independence of $d \rho_{1}, \cdots, d \rho_{\ell}$ at points of $M \cap U_{0}$, we also have the $\mathbb{R}$-linear independence of

$$
\left.\left(J d \rho_{1}\right)\right|_{T_{M}^{\mathbb{R}}}, \cdots,\left.\left(J d \rho_{\ell}\right)\right|_{T_{M}^{\mathbb{R}}}
$$

at points of $M \cap U_{0}$. The complex dimension of $T_{M}^{\mathbb{R}} \cap J T_{M}^{\mathbb{R}}$ is equal to $\operatorname{dim}_{\mathbb{C}} V-\ell$, which is $\geq 1$. The reason why the smallest complex submanifold germ $V$ of $\mathbb{C}^{n}$ at $P_{0}$ containing the germ of $M$ at $P_{0}$ satisfies condition (c) is that $T_{M}^{\mathbb{R}}$ is contained in the $J$-invariant vector space $\mathcal{N}$ at any point of $M$ and we can determine $V$ as the zero-set of holomorphic function germs on $\mathbb{C}^{n}$ at $P_{0}$ obtained by extending CR real-analytic functions on $M$ by using the condition of their annihilation by $\bar{\partial}$ to define the infinite jets of their extensions.

Condition (c) means that $V$ is tangential to $S$ at points of $M$. There are two possibilities. One is that $V$ is contained in $S$, in which case $S$ contains a local complex curve and we are done. The other possibility is that $V$ is not contained in $S$. We are going to assume the second possibility and derive a contradiction for the real-analytic case so that we can conclude in the real-analytic case that $S$ must contain a local complex curve. 
For clarity in the later discussion we digress at this point to say something about the well-known alternative description of the Levi form and also about the process of polarization.

(IV.6) Alternative Description of Levi Form. Recall the following formula of Cartan for exterior differentiation of differential forms

$$
\begin{gathered}
(p+1)(d \omega)\left(X_{1}, \cdots, X_{p+1}\right)=\sum_{i=1}^{p+1}(-1)^{i+1} X_{i}\left(\omega\left(X_{1}, \cdots, \hat{X}_{i}, \cdots, X_{p+1}\right)\right) \\
+\sum_{i<j}(-1)^{i+j} \omega\left(\left[X_{i}, X_{j}\right], X_{1}, \cdots, \hat{X}_{i}, \cdots, \hat{X}_{j}, \cdots, X_{p+1}\right),
\end{gathered}
$$

where $\omega$ is a differential $p$-form and $X_{1}, \cdots, X_{p+1}$ are vector fields and $\hat{X}_{i}$ and $\hat{X}_{j}$ indicate that $X_{i}$ and $X_{j}$ have been removed (see, for example, [He62, p.21, Formula (9)]). For the special case of $p=1$ we have

$$
2 d \omega\left(X_{1}, X_{2}\right)=X_{1}\left(\omega\left(X_{2}\right)\right)-X_{2}\left(\omega\left(X_{1}\right)\right)-\omega\left(\left[X_{1}, X_{2}\right]\right) .
$$

For a function $\rho$ and vector fields $\xi$ and $\eta$, by applying this to the case of $\omega=J d \rho$ (where $J$ is the almost-complex-structure operator), we get

$$
2 d J d \rho(\xi, \eta)=\xi(J d \rho(\eta))-\eta(J d \rho(\xi))-J d \rho([\xi, \eta]) .
$$

Note that $\partial \rho=\frac{1}{2}(1-\sqrt{-1} J) d \rho$ and $\bar{\partial} \rho=\frac{1}{2}(1+\sqrt{-1} J) d \rho$ so that

$$
\partial \bar{\partial} \rho=d \bar{\partial} \rho=\frac{1}{2} d d \rho+\frac{\sqrt{-1}}{2} d J d \rho=\frac{\sqrt{-1}}{2} d J d \rho
$$

and $d J d \rho=-2 \sqrt{-1} \partial \bar{\partial} \rho$. When $(d \rho)(\xi)=0$, we have

$$
(\partial \rho)(\xi)=\left(\frac{1}{2}(1-\sqrt{-1} J) d \rho\right)(\xi)=-\frac{\sqrt{-1}}{2}(J d \rho)(\xi) .
$$

When both $(d \rho)(\xi) \equiv 0$ and $(J d \rho)(\xi) \equiv 0$, we have

$$
J d \rho([\xi, \eta])=4 \sqrt{-1}(\partial \bar{\partial} \rho)(\xi, \eta) .
$$

When we compute the Levi form of $\rho$ we limit ourselves to vectors of type $(1,0)$ which are tangential to $\rho=0$. A vector $\xi$ of type $(1,0)$ means that $J(\xi)=\sqrt{-1} \xi$. Tangency of $\xi$ to $\rho=0$ means that $(d \rho)(\xi)=0$, which implies automatically $(J d \rho)(\xi)=(d \rho)(J \xi)=\sqrt{-1}(d \rho)(\xi)=0$, because by definition the operator $J$ acting on 1 -forms is the adjoint of the operator $J$ acting on tangent vectors. Likewise, for a vector $\bar{\xi}$ of type $(0,1)$ tangential to $\rho=0$ we have $(d \rho)(\bar{\xi})=0$ 
and $(J d \rho)(\bar{\xi})=(d \rho)(J \bar{\xi})=-\sqrt{-1}(d \rho)(\bar{\xi})=0$. Thus for vector fields $\xi$ and $\eta$ of type $(1,0)$ or $(1,0)$ tangential to $\rho=0$ we have

$$
J d \rho([\xi, \eta])=4 \sqrt{-1}(\partial \bar{\partial} \rho)(\xi, \eta) .
$$

(IV.7) Polarization. Let $Y$ be a CR submanifold of some open subset of $\mathbb{C}^{n}$. Let $\xi_{1}, \xi_{2}$ be real-valued vector fields in $T_{Y}^{\mathbb{R}} \cap J T_{Y}^{\mathbb{R}}$. The condition that $\xi_{j}$ is in $T_{Y}^{\mathbb{R}} \cap J T_{Y}^{\mathbb{R}}$ is equivalent to the condition that we can write $\xi_{j}=\tau_{j}+\overline{\tau_{j}}$ for some complex-valued vector fields $\tau_{j}$ in $T_{Y}^{(1,0)}$ for $j=1,2$. We have

$$
\left[\xi_{1}, \xi_{2}\right]=\left[\tau_{1}+\overline{\tau_{1}}, \tau_{2}+\overline{\tau_{2}}\right]=\left[\tau_{1}, \tau_{2}\right]+\left[\tau_{1}, \overline{\tau_{2}}\right]-\left[\tau_{2}, \overline{\tau_{1}}\right]+\left[\overline{\tau_{1}}, \overline{\tau_{2}}\right] .
$$

For the purpose of later computation of the Levi form, we now introduce the standard linear polarization process to express $\left[\tau_{2}, \overline{\tau_{1}}\right]$ in terms of $[\tau, \bar{\tau}]$ for some vector field $\tau$ of type $(1,0)$ tangential to $Y$ so that $\tau$ is expressed linearly and explicitly in terms of $\xi_{1}, \xi_{2}, J \xi_{1}, J \xi_{2}$ modulo $\mathbb{C} \otimes_{\mathbb{R}}\left(T_{Y}^{\mathbb{R}} \cap J T_{Y}^{\mathbb{R}}\right)=T_{Y}^{(1,0)} \oplus T_{Y}^{(0,1)}$. From

$$
\left[\tau_{1}+\tau_{2}, \overline{\tau_{1}+\tau_{2}}\right]=\left[\tau_{1}, \overline{\tau_{1}}\right]+\left[\tau_{1}, \overline{\tau_{2}}\right]+\left[\tau_{2}, \overline{\tau_{1}}\right]+\left[\tau_{2}, \overline{\tau_{2}}\right]
$$

we subtract the expression with $\tau_{2}$ changed to $-\tau_{2}$ to get

$$
\left[\tau_{1}+\tau_{2}, \overline{\tau_{1}+\tau_{2}}\right]-\left[\tau_{1}-\tau_{2}, \overline{\tau_{1}-\tau_{2}}\right]=2\left[\tau_{1}, \overline{\tau_{2}}\right]+2\left[\tau_{2}, \overline{\tau_{1}}\right] .
$$

Then we add to it $\sqrt{-1}$ times the expression which is obtained by replacing $\tau_{2}$ by $\sqrt{-1} \tau_{2}$ and we get $4\left[\tau_{1}, \overline{\tau_{2}}\right]$ equal to

$$
\begin{aligned}
& {\left[\tau_{1}+\tau_{2}, \overline{\tau_{1}+\tau_{2}}\right]-\left[\tau_{1}-\tau_{2}, \overline{\tau_{1}-\tau_{2}}\right]} \\
& +\sqrt{-1}\left(\left[\tau_{1}+\sqrt{-1} \tau_{2}, \overline{\tau_{1}+\sqrt{-1} \tau_{2}}\right]-\left[\tau_{1}-\sqrt{-1} \tau_{2}, \overline{\tau_{1}-\sqrt{-1} \tau_{2}}\right]\right) .
\end{aligned}
$$

Since $\left[\tau_{1}, \tau_{2}\right]$ is in $T_{Y}^{(1,0)}$ and $\left[\overline{\tau_{1}}, \overline{\tau_{2}}\right]$ is in $T_{Y}^{(0,1)}$, we conclude that modulo $\mathbb{C} \otimes_{\mathbb{R}}$ $\left(T_{Y}^{\mathbb{R}} \cap J T_{Y}^{\mathbb{R}}\right)=T_{Y}^{(1,0)} \oplus T_{Y}^{(0,1)}$ the Lie bracket $\left[\xi_{1}, \xi_{2}\right]$ is equal to $\left[\tau_{1}, \overline{\tau_{2}}\right]-\left[\tau_{2}, \overline{\tau_{1}}\right]$ which is in turn equal to $\frac{1}{4}$ times

$$
\begin{gathered}
{\left[\tau_{1}+\tau_{2}, \overline{\tau_{1}+\tau_{2}}\right]-\left[\tau_{1}-\tau_{2}, \overline{\tau_{1}-\tau_{2}}\right]} \\
+\sqrt{-1}\left(\left[\tau_{1}+\sqrt{-1} \tau_{2}, \overline{\tau_{1}+\sqrt{-1} \tau_{2}}\right]-\left[\tau_{1}-\sqrt{-1} \tau_{2}, \overline{\tau_{1}-\sqrt{-1} \tau_{2}}\right]\right) \\
-\left\{\left[\tau_{1}+\tau_{2}, \overline{\tau_{1}+\tau_{2}}\right]-\left[\tau_{2}-\tau_{1}, \overline{\tau_{2}-\tau_{1}}\right]\right. \\
\left.+\sqrt{-1}\left(\left[\tau_{2}+\sqrt{-1} \tau_{1}, \overline{\tau_{2}+\sqrt{-1} \tau_{1}}\right]-\left[\tau_{2}-\sqrt{-1} \tau_{1}, \overline{\tau_{2}-\sqrt{-1} \tau_{1}}\right]\right)\right\} \\
=2 \sqrt{-1}\left(\left[\tau_{1}+\sqrt{-1} \tau_{2}, \overline{\tau_{1}+\sqrt{-1} \tau_{2}}\right]-\left[\tau_{1}-\sqrt{-1} \tau_{2}, \overline{\tau_{1}-\sqrt{-1} \tau_{2}}\right]\right) .
\end{gathered}
$$


Thus modulo $\mathbb{C} \otimes_{\mathbb{R}}\left(T_{Y}^{\mathbb{R}} \cap J T_{Y}^{\mathbb{R}}\right)=T_{Y}^{(1,0)} \oplus T_{Y}^{(0,1)}$ the Lie bracket $\left[\xi_{1}, \xi_{2}\right]$ is equal to

$$
\frac{\sqrt{-1}}{2}\left(\left[\tau_{1}+\sqrt{-1} \tau_{2}, \overline{\tau_{1}+\sqrt{-1} \tau_{2}}\right]-\left[\tau_{1}-\sqrt{-1} \tau_{2}, \overline{\tau_{1}-\sqrt{-1} \tau_{2}}\right]\right)
$$

where

$$
\begin{aligned}
& \tau_{1}+\sqrt{-1} \tau_{2}=\frac{1}{2}\left(\xi_{1}+J \xi_{2}\right)+\frac{\sqrt{-1}}{2}\left(\xi_{2}-J \xi_{1}\right), \\
& \tau_{1}-\sqrt{-1} \tau_{2}=\frac{1}{2}\left(\xi_{1}-J \xi_{2}\right)-\frac{\sqrt{-1}}{2}\left(\xi_{2}+J \xi_{1}\right),
\end{aligned}
$$

because $\tau_{j}=\frac{1}{2}\left(\xi_{j}-\sqrt{-1} J \xi_{j}\right)$ for $j=1,2$. Suppose $\rho$ is a real-valued function in some neighborhood of $Y$. Then by (IV.6) we have

$$
\begin{aligned}
& J d \rho\left(\left[\tau_{1}, \tau_{2}\right]\right)=4 \sqrt{-1}(\partial \bar{\partial} \rho)\left(\tau_{1}, \tau_{2}\right)=0, \\
& J d \rho\left(\left[\overline{\tau_{1}}, \overline{\tau_{2}}\right]\right)=4 \sqrt{-1}(\partial \bar{\partial} \rho)\left(\overline{\tau_{1}}, \overline{\tau_{2}}\right)=0
\end{aligned}
$$

and as a consequence

$$
\begin{aligned}
(J d \rho)\left(\left[\xi_{1}, \xi_{2}\right]\right)=\frac{\sqrt{-1}}{2}(J & d \rho)\left(\left[\tau_{1}+\sqrt{-1} \tau_{2}, \overline{\tau_{1}+\sqrt{-1} \tau_{2}}\right]\right) \\
& -\frac{\sqrt{-1}}{2}(J d \rho)\left(\left[\tau_{1}-\sqrt{-1} \tau_{2}, \overline{\tau_{1}-\sqrt{-1} \tau_{2}}\right]\right) .
\end{aligned}
$$

When $\left|(J d \rho)\left(\left[\xi_{1}, \xi_{2}\right]\right)\right|=C$ for some $C>0$, we have $|(J d \rho)([\tau, \bar{\tau}])| \geq C$ for one of the following two values of $\tau$.

$$
\begin{aligned}
& \tau_{1}+\sqrt{-1} \tau_{2}=\frac{1}{2}\left(\xi_{1}+J \xi_{2}\right)+\frac{\sqrt{-1}}{2}\left(\xi_{2}-J \xi_{1}\right), \\
& \tau_{1}-\sqrt{-1} \tau_{2}=\frac{1}{2}\left(\xi_{1}-J \xi_{2}\right)-\frac{\sqrt{-1}}{2}\left(\xi_{2}+J \xi_{1}\right),
\end{aligned}
$$

(IV.8) Locating Holomorphic Direction at Which Precisely One Levi-Form Is Nonzero. After the above digression on the alternative description of the Levi form and the process of polarization, we now go back to the situation of the CR submanifold $M$ at the end of (IV.5). According to the construction of $M$ as an integral submanifold of $\tilde{\mathcal{N}}$ the tangent bundle $T_{M}^{\mathbb{R}}$ of $M$ is generated by iterated Lie brackets of vector fields of $\mathcal{N} \cap T_{E_{\infty}} \cap J T_{E_{\infty}}$ defined on $M$. Moreover, we have

$$
\mathcal{N} \cap T_{E_{\infty}} \cap J T_{E_{\infty}} \subset T_{M}^{\mathbb{R}} \cap J T_{M}^{\mathbb{R}}=\bigcap_{j=1}^{\ell} \operatorname{Ker}\left(\left.\left(J d \rho_{j}\right)\right|_{T_{M}^{\mathbb{R}}}\right) \subset T_{M}^{\mathbb{R}} .
$$

When we take vector fields in $\mathcal{N} \cap T_{E_{\infty}} \cap J T_{E_{\infty}}$ defined on $M$ and form their iterated Lie brackets in order to generate $T_{M}^{\mathbb{R}}$, there is a first time the vector field 
fails to be inside $T_{M}^{\mathbb{R}} \cap J T_{M}^{\mathbb{R}}=\bigcap_{j=1}^{\ell} \operatorname{Ker}\left(\left.\left(J d \rho_{j}\right)\right|_{T_{M}^{\mathbb{R}}}\right)$. Thus we can find realvalued vector fields $\xi_{1}, \xi_{2}$ in $T_{M}^{\mathbb{R}} \cap J T_{M}^{\mathbb{R}}=\bigcap_{j=1}^{\ell} \operatorname{Ker}\left(\left.\left(J d \rho_{j}\right)\right|_{T_{M}^{\mathbb{R}}}\right)$ defined on $M$ such that their Lie bracket $\left[\xi_{1}, \xi_{2}\right]$ is not in $T_{M}^{\mathbb{R}} \cap J T_{M}^{\mathbb{R}}=\bigcap_{j=1}^{\ell} \operatorname{Ker}\left(\left.\left(J d \rho_{j}\right)\right|_{T_{M}^{\mathbb{R}}}\right)$. There exists $1 \leq j \leq \ell$ such that $\left(J d \rho_{j}\right)\left(\left[\xi_{1}, \xi_{2}\right]\right)$ is nonzero. Without loss of generality we assume that $j=1$ so that $\left(J d \rho_{1}\right)\left(\left[\xi_{1}, \xi_{2}\right]\right)$ is nonzero. Since $\xi_{1}, \xi_{2}$ are both in $T_{M}^{\mathbb{R}} \cap J T_{M}^{\mathbb{R}}$, we can write $\xi_{j}=\tau_{j}+\overline{\tau_{j}}$ for some complex-valued vector fields $\tau_{j}$ in $T_{M}^{(1,0)}$ for $j=1,2$. As explained above in (IV.7), the polarization process gives us

$$
\begin{aligned}
\left(J d \rho_{1}\right)\left(\left[\xi_{1}, \xi_{2}\right]\right)=\frac{\sqrt{-1}}{2}( & \left.J d \rho_{1}\right)\left(\left[\tau_{1}+\sqrt{-1} \tau_{2}, \overline{\tau_{1}+\sqrt{-1} \tau_{2}}\right]\right) \\
& -\frac{\sqrt{-1}}{2}\left(J d \rho_{1}\right)\left(\left[\tau_{1}-\sqrt{-1} \tau_{2}, \overline{\tau_{1}-\sqrt{-1} \tau_{2}}\right]\right),
\end{aligned}
$$

where

$$
\begin{aligned}
& \tau_{1}+\sqrt{-1} \tau_{2}=\frac{1}{2}\left(\xi_{1}+J \xi_{2}\right)+\frac{\sqrt{-1}}{2}\left(\xi_{2}-J \xi_{1}\right), \\
& \tau_{1}-\sqrt{-1} \tau_{2}=\frac{1}{2}\left(\xi_{1}-J \xi_{2}\right)-\frac{\sqrt{-1}}{2}\left(\xi_{2}+J \xi_{1}\right),
\end{aligned}
$$

One of $\left(J d \rho_{1}\right)\left(\left[\tau_{1}+\sqrt{-1} \tau_{2}, \overline{\tau_{1}+\sqrt{-1} \tau_{2}}\right]\right)$ and $\left(J d \rho_{1}\right)\left(\left[\tau_{1}-\sqrt{-1} \tau_{2}, \overline{\tau_{1}-\sqrt{-1} \tau_{2}}\right]\right)$ must be nonzero at $P_{0}$. We can choose $\tau$ to be either $\tau_{1}+\sqrt{-1} \tau_{2}$ or $\tau_{1}-\sqrt{-1} \tau_{2}$ so that $\left(J d \rho_{1}\right)([\tau, \bar{\tau}])$ is nonzero at $P_{0}$. Since $\xi_{1}, \xi_{2}$ belong to $T_{M}^{\mathbb{R}} \cap J T_{M}^{\mathbb{R}}=$ $\bigcap_{j=1}^{\ell} \operatorname{Ker}\left(\left.\left(J d \rho_{j}\right)\right|_{T_{M}^{\mathbb{R}}}\right)$, it follows that $\left(\partial \rho_{j}\right)(\tau)=0$ at $P_{0}$ for $1 \leq j \leq \ell$.

Now for $2 \leq j \leq \ell$ we replace $\rho_{j}$ by

$$
\rho_{j}-\frac{\partial \rho_{j}([\tau, \bar{\tau}])}{\partial \rho_{1}([\tau, \bar{\tau}])} \rho_{1}
$$

so that we can assume without loss of generality that

$$
\begin{gathered}
0 \equiv\left(\partial \rho_{j}\right)([\tau, \bar{\tau}])=\left(\frac{1}{2}(1-\sqrt{-1} J) d \rho_{j}\right)([\tau, \bar{\tau}]) \\
=-\frac{\sqrt{-1}}{2}\left(J d \rho_{j}\right)([\tau, \bar{\tau}]) \quad \text { for } \quad 2 \leq j \leq \ell
\end{gathered}
$$

We can write

$$
\left.r\right|_{V}=\sum_{\nu_{1}+\cdots+\nu_{\ell}=k} \sigma_{\nu_{1}, \cdots, \nu_{\ell}}\left(\rho_{1}\right)^{\nu_{1}} \cdots\left(\rho_{\ell}\right)^{\nu_{\ell}}+O\left(\left(\sum_{j=1}^{\ell}\left(\rho_{j}\right)^{2}\right)^{\frac{k+1}{2}}\right)
$$


for some integer $k \geq 2$, where $\sigma_{\nu_{1}, \cdots, \nu_{\ell}}$ is a real-analytic function on $U_{0}$ (after shrinking $U_{0}$ as an open neighborhood of $P_{0}$ in $\mathbb{C}^{n}$ if necessary) and $\sigma_{\nu_{1}^{*}, \cdots, \nu_{\ell}^{*}}$ is nonzero at $P_{0}$ for some $\nu_{1}^{*}+\cdots+\nu_{\ell}^{*}=k$.

(IV.9) Argument of Different Vanishing Orders for Complex Hessian on the Complex Tangent Space Along Vector Fields Tangential or Normal to the Intersection with the Weakly Pseudoconvex Boundary. To make the argument more transparent and more understandable, we will first consider the special case $\ell=1$ so that $M=V \cap\left\{\rho_{1}=0\right\}$ and $V$ is a complex submanifold in some open neighborhood of some point $P_{0}$ of $M$. For this special case, for notational simplicity we drop the subscript 1 from $\rho_{1}$ and simply denote $\rho_{1}$ by $\rho$. By replacing $\rho$ by its product with a local nowhere zero real-analytic function we can assume without loss of generality that $r=\rho^{k}$ on $V$.

Let $m$ be the complex dimension of $V$. We choose a local holomorphic coordinate system $\left(z_{1}, \cdots, z_{n}\right)$ on the open neighborhood $U_{0}$ of $P_{0}$ in $\mathbb{C}^{n}$ centered at $P_{0}$ (after shrinking $U_{0}$ if necessary) such that $S \cap U_{0} \cap\left\{z_{m+1}=\cdots=z_{n}=0\right\}$ is regular and $V=\left\{z_{m+1}=\cdots=z_{n}=0\right\} \cap U_{0}$. Since our argument will be confined to an open neighborhood of $P_{0}$ in $\mathbb{C}^{n}$, for notational simplicity, by replacing $\mathbb{C}^{n}$ by $\mathbb{C}^{m+1}$ and $S$ by $S \cap\left\{z_{m+1}=\cdots=z_{n}=0\right\}$ we can assume without loss of generality that $n=m+1$ and we have the following setup.

(i) $d r=(0,0, \cdots, 0,1)$ at the origin so that the complex submanifold $V$ of the neighborhood $U_{0}$ of $P_{0}$ in $\mathbb{C}^{n}$ is an open subset of the complex tangent space of $S$ at the origin which is defined by $z_{n}=0$.

(ii) $r=x_{n}+O\left(|z|^{2}\right)$ near the origin, where $x_{n}$ is the real part of the coordinate $z_{n}$.

(iii) The intersection $M=V \cap S$ of $V$ and $S$ is a CR manifold whose complex tangent space $T_{M}^{\mathbb{R}} \cap J\left(T_{M}^{\mathbb{R}}\right)$ has positive complex dimension at every point of $M$.

(iv) $M$ is defined by $\rho=0$ in $V$ for some real-valued real-analytic function $\rho$ on $V$ such that $\left.r\right|_{V}=\rho^{k}$ for some positive integer $k$ and $d \rho$ is nowhere zero on $M$.

(v) For some nonzero tangent vector $\tau$ of type $(1,0)$ tangential to $M$ at the origin the value of the Levi form of $\rho$ at $\tau$ is nonzero. 
We are going to derive a contradiction. First we sketch the main idea of the argument. On $V$ we will introduce two vector fields of type $(1,0)$. One is tangential to $M$ at points of $M$ and the other is normal to $M$ at points of $M$. when we compute the complex Hessian of $\rho^{k}$ at these two vector fields of type $(1,0)$ on $V$, we get two different orders of vanishing as we approach $M$ from $V-M$, one of order $k-1$ and the other of order $k-2$. Because the touching order between $V$ and $S$ is $k$ along $M$, when we extend these two vector fields of type $(1,0)$ on $M$ to an open neighborhood of $P_{0}$ in $\mathbb{C}^{n}$ so that the two extensions are tangential to $S$ at points of $S$, the Levi forms of $r$ with respect to the two extensions give again the two different orders of vanishing as we approach $M$ from $S-M$. Since one of the two orders is odd, the weak pseudoconvexity of $S$ is violated, yielding a contradiction. Now we give below the details of this argument of different vanishing orders for the complex Hessian of $r$ on $V$ along vector fields tangential or normal to its intersection $M$ with the weakly pseudoconvex boundary $S$.

There is some open neighborhood $U_{1}$ of the origin 0 in $U_{0}$ on which

$$
r\left(z_{1}, \cdots, z_{n}\right)=\phi\left(z_{1}, \cdots, z_{n}\right) z_{n}+\overline{\phi\left(z_{1}, \cdots, z_{n}\right)} \overline{z_{n}}+\rho\left(z_{1}, \cdots, z_{n-1}\right)^{k}
$$

for some smooth complex-valued function $\phi\left(z_{1}, \cdots, z_{n}\right)$ on $U_{1}$, because on $V=$ $\left\{z_{n}=0\right\}$ the function $r$ is of the form $\rho^{k}$. Let $\xi$ be any smooth vector field of type $(1,0)$ on $U_{1}$ whose $n$-th component is $\xi_{n}$. Then

$$
\partial r=\partial \phi z_{n}+\phi d z_{n}+(\partial \bar{\phi}) \overline{z_{n}}+k \rho^{k-1} \partial \rho .
$$

$$
\begin{gathered}
\bar{\partial} r=\bar{\partial} \phi z_{n}+(\bar{\partial} \bar{\phi}) \overline{z_{n}}+\bar{\phi} d \overline{z_{n}}+k \rho^{k-1} \bar{\partial} \rho . \\
\partial \bar{\partial} r=\partial \bar{\partial} \phi z_{n}-\bar{\partial} \phi d z_{n}+\partial \bar{\partial} \bar{\phi} \overline{z_{n}}+\partial \bar{\phi} d \overline{z_{n}}+k(k-1) \rho^{k-2} \partial \rho \bar{\partial} \rho+k \rho^{k-1} \partial \bar{\partial} \rho .
\end{gathered}
$$$$
\langle\partial r, \xi\rangle=\langle\partial \phi, \xi\rangle z_{n}+\phi \xi_{n}+\langle\partial \bar{\phi}, \xi\rangle \overline{z_{n}}+k \rho^{k-1}\langle\partial \rho, \xi\rangle .
$$

$$
\begin{aligned}
\langle\partial \bar{\partial} r, \xi \wedge \bar{\xi}\rangle=\langle & \partial \bar{\partial} \phi, \xi \wedge \bar{\xi}\rangle z_{n}-\langle\bar{\partial} \phi, \bar{\xi}\rangle \xi_{n}+\langle\partial \bar{\partial} \bar{\phi}, \xi \wedge \bar{\xi}\rangle \overline{z_{n}}+\langle\partial \bar{\phi}, \xi\rangle \bar{\xi}_{n} \\
& +k(k-1) \rho^{k-2}\langle\partial \rho, \xi\rangle\langle\bar{\partial} \rho, \bar{\xi}\rangle+k \rho^{k-1}\langle\partial \bar{\partial} \rho, \xi \wedge \bar{\xi}\rangle
\end{aligned}
$$

At a point of $r=0$ in $U_{1}$ we have

$$
\phi z_{n}+\bar{\phi} \overline{z_{n}}+\rho^{k}=0 .
$$

Let $A$ and $B$ be respectively the real and imaginary parts of $2 \bar{\phi}$. Then $\phi=\frac{A-B i}{2}$ and $\bar{\phi}=\frac{A+B i}{2}$ so that

$$
\phi z_{n}+\bar{\phi} \overline{z_{n}}=A x_{n}+B y_{n}
$$


(where $y_{n}$ is the imaginary part of $z_{n}$ ) and at a point in $U_{1}$ we have

$$
A x_{n}+B y_{n}+\rho^{k}=0 \text {. }
$$

Since $d r=(0,0, \cdots, 0,1)$ at the origin, it follows that $\phi=\frac{1}{2}$ at the origin and $A=1$ and $B=0$ at the origin. Let $Y$ be the set defined by $y_{n}=0$. At any point of $S \cap Y \cap U$ where $A$ is nonzero, we have

$$
x_{n}=-\frac{\rho^{k}}{A}, \quad z_{n}=-\frac{\rho^{k}}{A}, \quad \overline{z_{n}}=-\frac{\rho^{k}}{A} .
$$

We can choose an open neighborhood $U$ of the origin 0 in $U_{1}$ of the form $U=$ $W \times G$ with $W \subset \mathbb{C}^{n-1}$ and $G \subset \mathbb{C}$ such that

(i) $A$ is nowhere zero on $U$ and for $Q \in W$ the set $G$ contains the point $z_{n}=-\frac{\rho(Q)^{k}}{A}$ and

(ii) $\phi+\left(\partial_{n} \phi\right) z_{n}+\left(\partial_{n} \bar{\phi}\right) \overline{z_{n}}$ is nowhere zero on $U_{1}$.

On $S \cap Y \cap U$ the two functions $z_{n}$ and $\overline{z_{n}}$ are of the order $O\left(\rho^{k}\right)$.

We now derive our contradiction by choosing $\xi$ in two different ways. The first way is to choose $\xi$ equal to $\tau$ at the origin. Since $\tau$ (from (IV.8)) is a vector of $\mathbb{C}^{n}$ of type $(1,0)$ at the origin which is tangential to $E=V \cap S$ and since $V=\left\{z_{n}=0\right\}$, it follows that the $n$-th component of the $n$-vector $\tau$ is zero. Since the differential $d \rho$ of the real-valued function $\rho$ on $V \cap U$ is nowhere zero at every point of $E=V \cap S$, we can extend $\tau$ to some smooth (1,0)-vector field $\xi=\left(\xi_{1}, \cdots, \xi_{n-1}\right)$ of $W_{1}$ for some open neighborhood $W_{1}$ of 0 in $W$ such that $\langle d \rho, \xi\rangle \equiv 0$ on $W_{1}$.

We regard $\xi_{j}=\xi_{j}\left(z_{1}, \cdots, z_{n-1}\right)$ as functions of $\left(z_{1}, \cdots, z_{n-1}, z_{n}\right) \in W_{1} \times G$ for $1 \leq j \leq n-1$ (which means denoting also by $\xi_{j}$ the composite of $\xi_{j}$ and the natural projection $W_{1} \times G \rightarrow W_{1}$ for $\left.1 \leq j \leq n-1\right)$. Since $\phi+\left(\partial_{n} \phi\right) z_{n}+\left(\partial_{n} \bar{\phi}\right) \overline{z_{n}}$ is nowhere zero on $U_{1}$, we can define $\xi_{n}$ on $W_{1} \times G$ by

$$
\xi_{n}=\frac{-1}{\phi+\left(\partial_{n} \phi\right) z_{n}+\left(\partial_{n} \bar{\phi}\right) \overline{z_{n}}}\left(\sum_{j=1}^{n-1}\left(\partial_{j} \phi\right) \xi_{j} z_{n}+\sum_{j=1}^{n-1}\left(\partial_{j} \bar{\phi}\right) \xi_{j} \overline{z_{n}}\right)
$$

so that the vector field $\xi=\left(\xi_{1}, \cdots, \xi_{n-1}, \xi_{n}\right)$ on $W_{1} \times G$ satisfies $\langle\partial r, \xi\rangle \equiv 0$ because of (IV.9.1). Since on $S \cap Y \cap U$ the two functions $z_{n}$ and $\overline{z_{n}}$ are of the order $O\left(\rho^{k}\right)$, it follows from (IV.9.3) that $\xi_{n}$ is of the order $O\left(\rho^{k}\right)$ on $S \cap Y \cap\left(W_{1} \times G\right)$. 
By (IV.9.2)

$$
\langle\partial \bar{\partial} r, \xi \wedge \bar{\xi}\rangle=k \rho^{k-1}\langle\partial \bar{\partial} \rho, \xi \wedge \bar{\xi}\rangle+O\left(\rho^{k}\right)
$$

on $S \cap Y \cap\left(W_{1} \times G\right)$. Since at the origin $\langle\partial \bar{\partial} \rho, \xi \wedge \bar{\xi}\rangle=\langle\partial \bar{\partial} \rho, \tau \wedge \bar{\tau}\rangle$ is nonzero and since $S$ is weakly pseudoconvex at every point of $S$, it follows that $k$ must be odd.

We now introduce our second way of choosing $\xi$ with the goal of deriving from it the conclusion that $k$ is even. We choose some smooth vector field $\left(\xi_{1}, \cdots, \xi_{n-1}\right)$ of type $(1,0)$ on some open neighborhood $W_{2}$ of 0 in $W$ such that $\left\langle d \rho,\left(\xi_{1}, \cdots, \xi_{n-1}\right)\right\rangle$ is nowhere zero on $W_{2}$. We now define $\xi_{n}$ on $W_{2} \times G$ by (IV.9.4)

$\xi_{n}=\frac{-1}{\phi+\left(\partial_{n} \phi\right) z_{n}+\left(\partial_{n} \bar{\phi}\right) \overline{z_{n}}}\left(\sum_{j=1}^{n-1}\left(\partial_{j} \phi\right) \xi_{j} z_{n}+\sum_{j=1}^{n-1}\left(\partial_{j} \bar{\phi}\right) \xi_{j} \overline{z_{n}}+k \rho^{k-1} \sum_{j=1}^{n-1}\left(\partial_{j} \rho\right) \xi_{j}\right)$

so that the vector field $\xi=\left(\xi_{1}, \cdots, \xi_{n-1}, \xi_{n}\right)$ on $W_{2} \times G$ satisfies $\langle\partial r, \xi\rangle \equiv 0$ because of (IV.9.1). Since on $S \cap Y \cap U$ the two functions $z_{n}$ and $\overline{z_{n}}$ are of the order $O\left(\rho^{k}\right)$, it follows from (IV.9.4) that $\xi_{n}$ is of the order $O\left(\rho^{k-1}\right)$ on $S \cap Y \cap\left(W_{2} \times G\right)$. By (IV.9.2)

$$
\langle\partial \bar{\partial} r, \xi \wedge \bar{\xi}\rangle=k(k-1) \rho^{k-2}\langle\partial \rho, \xi\rangle\langle\bar{\partial} \rho, \bar{\xi}\rangle+O\left(\rho^{k-1}\right)
$$

on $S \cap Y \cap\left(W_{2} \times G\right)$. Since at the origin $\left\langle d \rho,\left(\xi_{1}, \cdots, \xi_{n-1}\right)\right\rangle$ is nonzero and since $S$ is weakly pseudoconvex at every point of $S$, it follows that $k$ must be even. Thus we have a contradiction, because earlier we have the conclusion that $k$ must be odd.

(IV.10) Another Special Case to Illustrate the Argument of Different Tangential and Normal Vanishing Orders for Complex Hessian When Approaching CR Submanifold of Higher Holomorphic Codimension. We now consider another special case for the more general situation where locally $M$ is defined by real-valued realanalytic functions $\rho_{1}, \cdots, \rho_{\ell}$ on $V$ with $\ell>1$ and $\partial \rho_{1}, \cdots, \partial \rho_{\ell}$ are $\mathbb{C}$-linearly independent at points of $M$. We use this special case to further illustrate the argument of different tangential and normal vanishing orders for the complex Hessian. We first explain what this special case is.

As discussed above in (IV.8), there exist some $\tau \in T_{M}^{(1,0)}$ such that $\left(\partial \rho_{j}\right)(\tau)=0$ at $P_{0}$ for $1 \leq j \leq \ell$ and $\left(\partial \bar{\partial} \rho_{1}\right)(\tau, \bar{\tau})$ is nonzero but $\left(\partial \bar{\partial} \rho_{j}\right)(\tau, \bar{\tau})$ is zero for 
$2 \leq j \leq \ell$. We can write

$$
\left.r\right|_{V}=\sum_{\nu_{1}+\cdots+\nu_{\ell}=k} \sigma_{\nu_{1}, \cdots, \nu_{\ell}}\left(\rho_{1}\right)^{\nu_{1}} \cdots\left(\rho_{\ell}\right)^{\nu_{\ell}}+O\left(\left(\sum_{j=1}^{\ell}\left(\rho_{j}\right)^{2}\right)^{\frac{k+1}{2}}\right)
$$

for some integer $k \geq 2$, where $\sigma_{\nu_{1}, \cdots, \nu_{\ell}}$ is a real-analytic function on $U_{0}$ (after shrinking $U_{0}$ as an open neighborhood of $P_{0}$ in $\mathbb{C}^{n}$ if necessary) and $\sigma_{\nu_{1}^{*}, \cdots, \nu_{\ell}^{*}}$ is nonzero at $P_{0}$ for some $\nu_{1}^{*}+\cdots+\nu_{\ell}^{*}=k$. This special case which we now consider is when $\sigma_{\nu_{1}, \cdots, \nu_{\ell}}$ is nonzero at $P_{0}$ for some $\nu_{1}+\cdots+\nu_{\ell}=k$ with $\nu_{1} \neq 0$.

For this special case, just as for the case of $\ell=1$ we can find a smooth vector field $\xi$ of type $(1,0)$ in some open neighborhood of $P_{0}$ in $\mathbb{C}^{n}$ which are tangential to $\partial \Omega$ such that the value of $\xi$ at $P_{0}$ agrees with $\tau$. By computing the Levi form of $r$ at the vector field $\xi$ and its vanishing order at $M$ by using

$$
\left.r\right|_{V}=\sum_{\nu_{1}+\cdots+\nu_{\ell}=k} \sigma_{\nu_{1}, \cdots, \nu_{\ell}}\left(\rho_{1}\right)^{\nu_{1}} \cdots\left(\rho_{\ell}\right)^{\nu_{\ell}}+O\left(\left(\sum_{j=1}^{\ell}\left(\rho_{j}\right)^{2}\right)^{\frac{k+1}{2}}\right),
$$

as in the case of $\ell=1$ we can conclude that $k$ must be odd. Thus we have a contradiction. However, this argument depends on the additional assumption that $\sigma_{\nu_{1}, \cdots, \nu_{\ell}}$ is nonzero at $P_{0}$ for some $\nu_{1}+\cdots+\nu_{\ell}=k$ with $\nu_{1} \neq 0$ for a specially chosen set of defining functions $\rho_{1}, \cdots, \rho_{\ell}$.

Note that to rule out the case of an odd $k$, we do not need this additional assumption that $\sigma_{\nu_{1}, \cdots, \nu_{\ell}}$ is nonzero at $P_{0}$ for some $\nu_{1}+\cdots+\nu_{\ell}=k$ with $\nu_{1} \neq 0$. There is also another way to rule out the case of an odd $k$ by using bounded strictly plurisubharmonic exhaustion functions for weakly pseudoconvex domains in the following way.

(IV.11) Handling the Case of Odd Vanishing Order by Using Bounded Strictly Plurisubharmonic Exhaustion Functions for Weakly Pseudoconvex Domains. First let us introduce the following trivial statement about the vanishing order of a negative subharmonic function at a boundary segment, which is related to Hopf's lemma or the strong maximum principle [GT83, p.34, Lemma 3.4].

(IV.11.1) Let $D$ be a connected open subset of $\mathbb{C}$ and $C$ is a smooth connected curve in $D$ defined by $\rho=0$ with $d \rho$ nowhere zero at points of $C$ such that $D-C$ consists of two nonempty components $W_{1}$ and $W_{2}$ with $\rho<0$ on $W_{1}$. Let $\eta>1$ 
and $\varphi$ be a smooth negative subharmonic function on $W_{1}$. Then it is impossible to write $-\varphi=(-\rho)^{\eta}$ on $W_{1}$.

The reason is as follows. We compute

$$
\begin{gathered}
\bar{\partial}(-\varphi)=-\eta(-\rho)^{\eta-1} \bar{\partial} \rho \\
\partial \bar{\partial}(-\varphi)=\eta(\eta-1)(-\rho)^{\eta-2} \partial \rho \bar{\partial} \rho-\eta(-\rho)^{\eta-1} \partial \bar{\partial} \rho .
\end{gathered}
$$

Since $\partial \bar{\partial} \varphi \geq 0$ on $W_{1}$, it follows that

$$
0 \geq \partial \bar{\partial}(-\varphi)=\eta(\eta-1)(-\rho)^{\eta-2} \partial \rho \bar{\partial} \rho-\eta(-\rho)^{\eta-1} \partial \bar{\partial} \rho
$$

and

$$
\partial \bar{\partial} \rho \geq \partial \bar{\partial}(-\varphi)=\frac{\eta-1}{-\rho} \partial \rho \bar{\partial} \rho
$$

which is a contradiction, because the left-hand side evaluated at a point of $W_{1}$ stays bounded as the point approaches some point of $C$ but the right-hand side evaluated at the same point becomes $\infty$ as the point approaches some point of C.

We now recall the following theorem of Diederich-Fornaess on bounded strictly plurisubharmonic exhaustion functions for weakly pseudoconvex domains [DF77, p.133, Remark b].

Let $\Omega$ be a domain in $\mathbb{C}^{n}$ and $P_{0}$ belong to the boundary of $\Omega$ so that for some open neighborhood $D$ of $P_{0}$ in $\mathbb{C}^{n}$ the boundary of $\Omega \cap D$ in $D$ is smooth and weakly pseudoconvex. Let $\delta$ be the distance function from a point of $\Omega$ to $\mathbb{C}^{n}-\Omega$. Let $\psi$ be a smooth strictly plurisubharmonic function on $\mathbb{C}^{n}$ (or just defined on some open neighborhood of some point of $\partial \Omega$ in $\left.\mathbb{C}^{n}\right)$. Then for any choice of $0<\gamma<1$ there is a suitable choice of a sufficiently small $L>0$ such that the complex Hessian $\partial \bar{\partial}\left(-\delta^{\gamma} e^{-L \psi}\right)$ is strictly positive on $\Omega \cap D^{\prime}$ for some open neighborhood $D^{\prime}$ of $P_{0}$ in $D$.

Suppose we have the case of an odd $k$ in the following expansion which we would like to rule out.

$$
\left.r\right|_{V}=\sum_{\nu_{1}+\cdots+\nu_{\ell}=k} \sigma_{\nu_{1}, \cdots, \nu_{\ell}}\left(\rho_{1}\right)^{\nu_{1}} \cdots\left(\rho_{\ell}\right)^{\nu_{\ell}}+O\left(\left(\sum_{j=1}^{\ell}\left(\rho_{j}\right)^{2}\right)^{\frac{k+1}{2}}\right)
$$

for some integer $k \geq 2$, where $\sigma_{\nu_{1}, \cdots, \nu_{\ell}}$ is a real-analytic function on $U_{0}$ (after shrinking $U_{0}$ as an open neighborhood of $P_{0}$ in $\mathbb{C}^{n}$ if necessary) and $\sigma_{\nu_{1}^{*}, \cdots, \nu_{\ell}^{*}}$ 
is nonzero at $P_{0}$ for some $\nu_{1}^{*}+\cdots+\nu_{\ell}^{*}=k$. Assume that $k$ is odd. We can find a tangent vector $\eta$ of $V$ at the point $P_{0}$ of $M$ normal to $M$ such that $J \eta$ is tangential to $M$ and the $k$-derivative of $r$ in the direction of $\eta$ is nonzero. Let $C$ be local complex curve in $\mathbb{C}^{n}$ through $P_{0}$ such that the complex tangent vector to $C$ of type $(1,0)$ at $P_{0}$ is equal to $\eta-\sqrt{-1} J \eta$ and $C \cap \partial \Omega \subset M$ and $C \cap M$ is a regular curve in $C$. Since $k$ is odd, after replacing $C$ by an open neighborhood of $P_{0}$ in $C$ we can assume without loss of generality that $C-M$ consists of two nonempty connected components $C \cap \Omega$ and $C-\bar{\Omega}$.

Let $\kappa=-\delta^{\gamma} e^{-L \psi}$ and we restrict $\kappa$ to $C \cap \Omega$. Let $\phi$ be a smooth function on $C$ whose zero-set is $C \cap M$ and which is negative on $C \cap \Omega$ with $d \phi$ nowhere zero on $C \cap M$. Since $-\kappa$ is equal to $\sigma(-r)^{\gamma}=\tilde{\sigma}(-\phi)^{k \gamma}$ on $C \cap \Omega$ for some positive-valued smooth functions $\sigma$ and $\tilde{\sigma}$ on $C$ (after replacing $C$ by an open neighborhood of $P_{0}$ in $C$ if necessary), from the plurisubharmonicity of $\kappa$ on $\Omega$ we have a contradiction to (IV.11.1) when $0<\gamma<1$ is chosen to satisfy $k \gamma>1$, because $\left.\kappa\right|_{C \cap \Omega}$ is subharmonic on $C \cap \Omega$ and $-\left.\kappa\right|_{C \cap \Omega}$ is equal to $\left(-\tilde{\sigma}^{\frac{1}{k \gamma}} \phi\right)^{k \gamma}$ and $\tilde{\sigma}^{\frac{1}{k \gamma}} \phi$ is smooth on $C$ and is 0 at $C \cap M$ and $d \tilde{\sigma}^{\frac{1}{k \gamma}} \phi$ is nowhere zero on $M$. This argument avoids the process in (IV.10) of constructing the analog of the second vector field, at the end of (IV.9), of type $(1,0)$ in a neighborhood of $P_{0}$ in $\mathbb{C}^{n}$ tangential to $S$ and not tangential to $M$ at $P_{0}$.

(IV.12) Handling the Case of Even Vanishing Order by Stratification According to Iterated Lie Brackets. We now deal with the general case by choosing the set of defining functions $\rho_{1}, \cdots, \rho_{\ell}$ by stratification according to iterated Lie brackets. Recall that iterated Lie brackets of vector fields on $E_{\infty}$ with coefficients in $\mathcal{N}^{(\infty)}=\mathcal{N} \cap T_{E_{\infty}}^{\mathbb{R}} \cap J T_{E_{\infty}}^{\mathbb{R}}$ generate the distribution $\tilde{\mathcal{N}}$ and $M$ is an integral submanifold of $E_{\infty}$ whose tangent space at every point is equal to the subspace distribution $\tilde{\mathcal{N}}$ at that point. Because of the Jacobi identity for the Lie brackets of three vector fields, we can select vector fields $\tau_{0}, \tau_{1}, \cdots, \tau_{\ell}$ on $E_{\infty}$ with values in $\mathcal{N}^{(\infty)}=\mathcal{N} \cap T_{E_{\infty}}^{\mathbb{R}} \cap J T_{E_{\infty}}^{\mathbb{R}}$ such that inductively, $\tilde{\tau}_{1}=\left[\tau_{0}, \tau_{1}\right]$ and $\tilde{\tau}_{j}=\left[\tilde{\tau}_{j-1}, \tau_{j}\right]$ for $2 \leq j \leq \ell$ and $\tilde{\tau}_{j}\left(P_{0}\right)$ is not spanned by $\left(\mathcal{N}^{(\infty)}\right)_{P_{0}}, \tilde{\tau}_{1}\left(P_{0}\right), \cdots, \tilde{\tau}_{j-1}\left(P_{0}\right)$ for $1 \leq j \leq \ell$. We now choose $\rho_{1}, \cdots, \rho_{\ell}$ such that, modulo $\left(\mathcal{N}^{(\infty)}\right)_{P_{0}}$, the 1 -forms $\left(J d \rho_{j}\right)\left(P_{0}\right)$ at $P_{0}$ for $1 \leq j \leq \ell$, when restricted to the tangent space $T_{M, P_{0}}^{\mathbb{R}}$ of $M$ at $P_{0}$ precisely form a dual basis for $\tilde{\tau}_{1}\left(P_{0}\right), \cdots, \tilde{\tau}_{\ell}\left(P_{0}\right)$. In other words, the $\mathbb{R}$-linear functionals defined by $\left(J d \rho_{j}\right)\left(P_{0}\right)$ at $P_{0}$ for $1 \leq j \leq \ell$ on the quotient 
space $T_{M, P_{0}}^{\mathbb{R}} /\left(\mathcal{N}^{(\infty)}\right)_{P_{0}}$ form the dual basis of the elements in $T_{M, P_{0}}^{\mathbb{R}} /\left(\mathcal{N}^{(\infty)}\right)_{P_{0}}$ induced by $\tilde{\tau}_{1}\left(P_{0}\right), \cdots, \tilde{\tau}_{\ell}\left(P_{0}\right)$.

Let $\xi_{j}=\frac{1}{2}\left(\tilde{\tau}_{j-1}-\sqrt{-1} J \tilde{\tau}_{j-1}\right)$ and $\eta_{j}=\frac{1}{2}\left(\tau_{j}-\sqrt{-1} J \tau_{j}\right)$ on $M$ for $1 \leq j \leq \ell$ so that both $\xi_{j}$ and $\eta_{j}$ are of type $(1,0)$ tangential to $V$ with the real part of $\xi_{j}$ being $\frac{1}{2} \tilde{\tau}_{j}$ and the real part of $\eta_{j}$ being $\frac{1}{2} \tau_{j}$. Take $2 \leq j \leq \ell$. As verified above in (IV.7), from $\tilde{\tau}_{j-1}=\xi_{j}+\overline{\xi_{j}}$ and $\tau_{j}=\eta_{j}+\overline{\eta_{j}}$ we get

$$
\left[\tilde{\tau}_{j-1}, \tau_{j}\right]=\frac{\sqrt{-1}}{2}\left(\left[\xi_{j}+\sqrt{-1} \eta_{j}, \overline{\xi_{j}+\sqrt{-1} \eta_{j}}\right]-\left[\xi_{j}-\sqrt{-1} \eta_{j}, \overline{\xi_{j}-\sqrt{-1} \eta_{j}}\right]\right)
$$

modulo $\mathbb{C} \otimes_{\mathbb{R}}\left(T_{M}^{\mathbb{R}} \cap J T_{M}^{\mathbb{R}}\right)=T_{M}^{(1,0)} \oplus T_{M}^{(0,1)}$, where

$$
\begin{aligned}
& \xi_{j}+\sqrt{-1} \eta_{j}=\frac{1}{2}\left(\tilde{\tau}_{j-1}+J \tau_{j}\right)+\frac{\sqrt{-1}}{2}\left(\tau_{j}-J \tilde{\tau}_{j-1}\right), \\
& \xi_{j}-\sqrt{-1} \eta_{j}=\frac{1}{2}\left(\tilde{\tau}_{j-1}-J \tau_{j}\right)-\frac{\sqrt{-1}}{2}\left(\tau_{j}+J \tilde{\tau}_{j-1}\right) .
\end{aligned}
$$

At the point $P_{0}$ we have

$$
\begin{gathered}
1=\left(J d \rho_{j}\right)\left(\tilde{\tau}_{j}\right) \\
=\frac{\sqrt{-1}}{2}\left(J d \rho_{j}\right)\left(\left[\xi_{j}+\sqrt{-1} \eta_{j}, \overline{\xi_{j}+\sqrt{-1} \eta_{j}}\right]-\left[\xi_{j}-\sqrt{-1} \eta_{j}, \overline{\xi_{j}-\sqrt{-1} \eta_{j}}\right]\right)
\end{gathered}
$$

and at least one of

$$
\left(J d \rho_{j}\right)\left(\left[\xi_{j}+\sqrt{-1} \eta_{j}, \overline{\xi_{j}+\sqrt{-1} \eta_{j}}\right]\right) \quad \text { and } \quad\left(J d \rho_{j}\right)\left(\left[\xi_{j}-\sqrt{-1} \eta_{j}, \overline{\xi_{j}-\sqrt{-1} \eta_{j}}\right]\right)
$$

has absolute value at least 1 and is nonzero at $P_{0}$. We set $\zeta_{j}$ to be one of the two possibilities

$$
\begin{aligned}
& \xi_{j}+\sqrt{-1} \eta_{j}=\frac{1}{2}\left(\tilde{\tau}_{j-1}+J \tau_{j}\right)+\frac{\sqrt{-1}}{2}\left(\tau_{j}-J \tilde{\tau}_{j-1}\right), \\
& \xi_{j}-\sqrt{-1} \eta_{j}=\frac{1}{2}\left(\tilde{\tau}_{j-1}-J \tau_{j}\right)-\frac{\sqrt{-1}}{2}\left(\tau_{j}+J \tilde{\tau}_{j-1}\right) .
\end{aligned}
$$

so that $\left|\left(J d \rho_{j}\right)\left(\left[\zeta_{j}, \overline{\zeta_{j}}\right]\right)\right| \geq 1$ at $P_{0}$. From the way we define the 1 -jet of $\rho_{j}$ at $P_{0}$ we know that among the following vectors

$$
\begin{gathered}
\tau_{0}, \tau_{1}, \cdots, \tau_{\ell}, J \tau_{0}, J \tau_{1}, \cdots, J \tau_{\ell}, \\
\tilde{\tau}_{1}, \cdots, \tilde{\tau}_{\ell}, J \tilde{\tau}_{1}, \cdots, J \tilde{\tau}_{\ell}
\end{gathered}
$$

at $P_{0}$ the only one at which $d \rho_{j}$ is nonzero is $J \tilde{\tau}_{j}$ where the value of $d \rho_{j}$ is 1 , because the vectors

$$
\tau_{0}, \tau_{1}, \cdots, \tau_{\ell}, J \tau_{0}, J \tau_{1}, \cdots, J \tau_{\ell}, \tilde{\tau}_{1}, \cdots, \tilde{\tau}_{\ell}
$$


all belong to the tangent space $T_{M, P_{0}}^{\mathbb{R}}$ of $M$ at $P_{0}$ which is equal to $\left(\mathcal{N}^{(\infty)}\right)_{P_{0}}$ and $\rho_{j}$ vanishes on $M$ and because the $\mathbb{R}$-linear functionals defined by

$$
\left(J d \rho_{1}\right)\left(P_{0}\right), \cdots,\left(J d \rho_{\ell}\right)\left(P_{0}\right)
$$

at $P_{0}$ on the quotient space $T_{M, P_{0}}^{\mathbb{R}} /\left(\mathcal{N}^{(\infty)}\right)_{P_{0}}$ form the dual basis of the elements in $T_{M, P_{0}}^{\mathbb{R}} /\left(\mathcal{N}^{(\infty)}\right)_{P_{0}}$ induced by $\tilde{\tau}_{1}\left(P_{0}\right), \cdots, \tilde{\tau}_{\ell}\left(P_{0}\right)$. Hence $\left(d \rho_{j}\right)\left(\zeta_{p}\right)$ at $P_{0}$ is 0 for $j \neq p-1$.

For later use we need a slight variation of the above discussion. Take a positive number $A$. From $\tilde{\tau}_{j-1}=\xi_{j}+\overline{\xi_{j}}$ and $\tau_{j}=\eta_{j}+\overline{\eta_{j}}$ we get

$A \tilde{\tau}_{j}=\left[\tilde{\tau}_{j-1}, A \tau_{j}\right]=\frac{1}{2}\left(\left[\xi_{j}+\sqrt{-1} A \eta_{j}, \overline{\xi_{j}+i A \eta_{j}}\right]-\left[\xi_{j}-\sqrt{-1} A \eta_{j}, \overline{\xi_{j}-\sqrt{-1} A \eta_{j}}\right]\right)$,

where

$$
\begin{aligned}
& \xi_{j}+\sqrt{-1} A \eta_{j}=\frac{1}{2}\left(\tilde{\tau}_{j-1}+A J \tau_{j}\right)-\frac{\sqrt{-1}}{2}\left(\tilde{\tau}_{j-1}+A J \tau_{j}\right), \\
& \xi_{j}-\sqrt{-1} A \eta_{j}=\frac{1}{2}\left(\tilde{\tau}_{j-1}-A J \tau_{j}\right)-\frac{\sqrt{-1}}{2}\left(\tilde{\tau}_{j-1}-A J \tau_{j}\right) .
\end{aligned}
$$

At the point $P_{0}$ we have

$$
\begin{gathered}
A=A\left(J d \rho_{j}\right)\left(\tilde{\tau}_{j}\right)=\left(J d \rho_{j}\right)\left(A \tilde{\tau}_{j}\right)=\left(J d \rho_{j}\right)\left(\left[\tilde{\tau}_{j-1}, A \tau_{j}\right]\right) \\
=\frac{\sqrt{-1}}{2}\left(J d \rho_{j}\right)\left(\left[\xi_{j}+\sqrt{-1} A \eta_{j}, \overline{\xi_{j}+\sqrt{-1} A \eta_{j}}\right]-\left[\xi_{j}-\sqrt{-1} A \eta_{j}, \overline{\xi_{j}-\sqrt{-1} A \eta_{j}}\right]\right)
\end{gathered}
$$

and at least one of

$$
\left(J d \rho_{j}\right)\left(\left[\xi_{j}+\sqrt{-1} A \eta_{j}, \overline{\xi_{j}+\sqrt{-1} A \eta_{j}}\right]\right) \text { and }\left(J d \rho_{j}\right)\left(\left[\xi_{j}-\sqrt{-1} A \eta_{j}, \overline{\xi_{j}-\sqrt{-1} A \eta_{j}}\right]\right)
$$

has absolute value at least $A$ at $P_{0}$. We set $\zeta_{j, A}$ to be one of the two possibilities

$$
\begin{aligned}
& \xi_{j}+\sqrt{-1} A \eta_{j}=\frac{1}{2}\left(\tilde{\tau}_{j-1}+A J \tau_{j}\right)+\frac{\sqrt{-1}}{2}\left(A \tau_{j}-J \tilde{\tau}_{j-1}\right), \\
& \xi_{j}-\sqrt{-1} A \eta_{j}=\frac{1}{2}\left(\tilde{\tau}_{j-1}-A J \tau_{j}\right)-\frac{\sqrt{-1}}{2}\left(A \tau_{j}+J \tilde{\tau}_{j-1}\right)
\end{aligned}
$$

so that $\left|\left(J d \rho_{j}\right)\left(\left[\zeta_{j, A}, \overline{\zeta_{j, A}}\right]\right)\right| \geq A$ at $P_{0}$. Note that when $A=1$ we have $\zeta_{j, A}=\zeta_{j}$ so that for any value of $A>1$ the vector $\zeta_{j, A}-\zeta_{j}$ is equal to

$$
\pm\left(\frac{(A-1)}{2} J \tau_{j}+\frac{\sqrt{-1}(A-1)}{2} \tau_{j}\right) .
$$


From the way we define the 1 -jet of $\rho_{j}$ at $P_{0}$ we know that among the following vectors

$$
\begin{gathered}
\tau_{0}, \tau_{1}, \cdots, \tau_{\ell}, J \tau_{0}, J \tau_{1}, \cdots, J \tau_{\ell}, \\
\tilde{\tau}_{1}, \cdots, \tilde{\tau}_{\ell}, J \tilde{\tau}_{1}, \cdots, J \tilde{\tau}_{\ell}
\end{gathered}
$$

at $P_{0}$ the only one at which $d \rho_{j}$ is nonzero is $J \tilde{\tau}_{j}$ where the value of $d \rho_{j}$ is 1 , because the vectors

$$
\tau_{0}, \tau_{1}, \cdots, \tau_{\ell}, J \tau_{0}, J \tau_{1}, \cdots, J \tau_{\ell}, \tilde{\tau}_{1}, \cdots, \tilde{\tau}_{\ell}
$$

all belong to the tangent space $T_{M, P_{0}}^{\mathbb{R}}$ of $M$ at $P_{0}$ which is equal to $\left(\mathcal{N}^{(\infty)}\right)_{P_{0}}$ and $\rho_{j}$ vanishes on $M$ and because the $\mathbb{R}$-linear functionals defined by

$$
\left(J d \rho_{1}\right)\left(P_{0}\right), \cdots,\left(J d \rho_{\ell}\right)\left(P_{0}\right)
$$

at $P_{0}$ on the quotient space $T_{M, P_{0}}^{\mathbb{R}} /\left(\mathcal{N}^{(\infty)}\right)_{P_{0}}$ form the dual basis of the elements in $T_{M, P_{0}}^{\mathbb{R}} /\left(\mathcal{N}^{(\infty)}\right)_{P_{0}}$ induced by $\tilde{\tau}_{1}\left(P_{0}\right), \cdots, \tilde{\tau}_{\ell}\left(P_{0}\right)$. Hence $\left(d \rho_{j}\right)\left(\zeta_{p}\right)$ at $P_{0}$ is 0 for $j \neq p-1$. Moreover, at $P_{0}$

$$
\left(d \rho_{j}\right)\left(\zeta_{p, A}-\zeta_{p}\right)=\left(d \rho_{j}\right)\left( \pm\left(\frac{(A-1)}{2} J \tau_{j}+\frac{\sqrt{-1}(A-1)}{2} \tau_{j}\right)\right)=0 .
$$

Thus $\left(d \rho_{j}\right)\left(\zeta_{p, A}\right)=\left(d \rho_{j}\right)\left(\zeta_{p}\right)$ is independent of $A$ for all $p$ and $j$.

Let $1<q \leq \ell$ be the minimum such that $\nu_{q} \geq 1$ and $\nu_{q}+\cdots+\nu_{\ell}=k$ and $\sigma_{0, \cdots, 0, \nu_{q}, \cdots, \nu_{\ell}}\left(P_{0}\right) \neq 0$. Since we have $\zeta_{q, A} \rho_{j}=0$ at $P_{0}$ for $j \neq q-1$, the term of lowest vanishing order at $P_{0}$ which we can get is $k-1$ and either come with the factors $\zeta_{q, A} \overline{\zeta_{q, A}} \rho_{j}$ at $P_{0}$ from

$$
\sum_{\nu_{1}+\cdots+\nu_{\ell}=k} \sigma_{\nu_{1}, \cdots, \nu_{\ell}}\left(\rho_{1}\right)^{\nu_{1}} \cdots\left(\rho_{\ell}\right)^{\nu_{\ell}}
$$

or come with the factor $\zeta_{q, A} \rho_{q-1} \overline{\zeta_{q, A}} \rho_{q-1}$ at $P_{0}$ from

$$
\sum_{\nu_{1}+\cdots+\nu_{\ell}=k+1} \sigma_{\nu_{1}, \cdots, \nu_{\ell}}\left(\rho_{1}\right)^{\nu_{1}} \cdots\left(\rho_{\ell}\right)^{\nu_{\ell}}
$$

in the expansion of $\left.r\right|_{V}$. The sum of all these terms of vanishing order $k-1$ at $P_{0}$ is identically zero only when

$$
\begin{gathered}
\sum_{\nu_{q}+\cdots+\nu_{\ell}=k} \sigma_{0, \cdots, 0, \nu_{q}, \cdots, \nu_{\ell} \nu_{q}}\left(\zeta_{q, A} \overline{\zeta_{q, A}} \rho_{q}\right)\left(\rho_{q}\right)^{\nu_{q}-1}\left(\rho_{q+1}\right)^{\nu_{q+1}} \cdots\left(\rho_{\ell}\right)^{\nu_{\ell}} \\
=-\sum_{\nu_{1}+\cdots+\nu_{\ell}=k+1} \sigma_{\nu_{1}, \cdots, \nu_{\ell}} \nu_{q-1}\left(\nu_{q-1}-1\right)\left(\zeta_{q, A} \rho_{q-1}\right)\left(\overline{\zeta_{q, A}} \rho_{q-1}\right) .
\end{gathered}
$$




$$
\cdot\left(\left(\rho_{1}\right)^{\nu_{1}} \cdots\left(\rho_{q-2}\right)^{\nu_{q-2}}\left(\rho_{q-1}\right)^{\nu_{q-1}-2}\left(\rho_{q+1}\right)^{\nu_{q+1}} \cdots\left(\rho_{\ell}\right)^{\nu_{\ell}}\right) \cdot
$$

Since the contradiction comes from the change of the sign of the Levi Form of $S=\partial \Omega$ when we approach $M$ from along some appropriate path in $S=\partial \Omega$ which corresponds to a path in $V$ up to order $k$, we have trouble only when for any choice of $\nu_{q} \geq 1, \nu_{q+1} \geq 0, \cdots, \nu_{\ell} \geq 0$ with $\nu_{q}+\cdots+\nu_{\ell}=k$ we have

$$
\begin{aligned}
& \sigma_{0, \cdots, 0, \nu_{q}, \cdots, \nu_{\ell} \nu_{q}}\left(\zeta_{q, A} \overline{\zeta_{q, A}} \rho_{q}\right) \\
& \quad=-\sum_{1 \leq i \leq j<q} \sigma_{0, \cdots, 0, \nu_{q-1}+2, \nu_{q}-1, \nu_{q+1} \cdots, \nu_{\ell}} \nu_{q-1}\left(\nu_{q-1}-1\right)\left(\zeta_{q, A} \rho_{q-1}\right)\left(\overline{\zeta_{q, A}} \rho_{q-1}\right)
\end{aligned}
$$

at the point $P_{0}$ of $M$ for any choice of $\nu_{q} \geq 1, \nu_{q+1} \geq 0, \cdots, \nu_{\ell} \geq 0$ with $\nu_{q}+$ $\cdots+\nu_{\ell}=k$, which is the same as

$$
\begin{aligned}
& A^{2} \sigma_{0, \cdots, 0, \nu_{q}, \cdots, \nu_{\ell}} \nu_{q}\left(\zeta_{q} \overline{\zeta_{q}} \rho_{q}\right) \\
& =-\sum_{1 \leq i \leq j<q} \sigma_{0, \cdots, 0, \nu_{q-1}+2, \nu_{q}-1, \nu_{q+1} \cdots, \nu_{\ell}} \nu_{q-1}\left(\nu_{q-1}-1\right)\left(\zeta_{q} \rho_{q-1}\right)\left(\overline{\zeta_{q}} \rho_{q-1}\right) \text {, }
\end{aligned}
$$

because $\left(\zeta_{q, A} \overline{\zeta_{q, A}} \rho_{q}\right)\left(P_{0}\right)=A^{2}\left(\zeta_{q} \overline{\zeta_{q}} \rho_{q}\right)\left(P_{0}\right)$ and $\left(\zeta_{q, A} \rho_{q-1}\right)\left(P_{0}\right)=\left(\zeta_{q} \rho_{q-1}\right)\left(P_{0}\right)$ and $\left(\overline{\zeta_{q, A}} \rho_{q-1}\right)\left(P_{0}\right)=\left(\overline{\zeta_{q}} \rho_{q-1}\right)\left(P_{0}\right)$. Since $\left(\zeta_{q} \overline{\zeta_{q}} \rho_{q}\right)\left(P_{0}\right) \neq 0$, this trouble can simply be handled with the choice of a sufficiently large $A$.

Finally, in order to get a contradiction from the evenness of the vanishing order $k$ of $\left.r\right|_{V \cap U}$ at $M \cap U$, we construct

(i) a real-analytic curve $\Gamma_{S}$ in $S \cap U$ containing $P_{0}$ which is transversal to $M$

(ii) a real-analytic curve $\Gamma_{V}$ in $V \cap U$ containing $P_{0}$ which is transversal to $M$

(iii) a smooth bijection $\Psi$ from $\Gamma_{V}$ to $\Gamma_{S}$,

(iv) a vector field $\zeta_{S}$ of type $(1,0)$ tangential to $S$ defined only at points of the curve $\Gamma_{S}$ and smooth along $\Gamma_{S}$ whose value at $P_{0}$ is $\zeta_{q, A}$, and

(v) a vector field $\zeta_{V}$ of type $(1,0)$ tangential to $V$ defined only at points of the curve $\Gamma_{V}$ and smooth along $\Gamma_{V}$ whose value at $P_{0}$ is $\zeta_{q, A}$

such that

(a) the distance between $P \in \Gamma_{V}$ and $\Psi(P) \in \Gamma_{S}$ is of order of $\left(\operatorname{dist}_{\Gamma_{V}}\left(P, P_{0}\right)\right)^{k}$, where $\operatorname{dist}_{\Gamma_{V}}\left(P, P_{0}\right)$ is the distance between $P$ and $P_{0}$ along $\Gamma_{V}$, and

(a) the difference of the value of $\zeta_{V}$ at $P \in \Gamma_{V}$ and the value of $\zeta_{S}$ at $\Psi(P) \in$ $\Gamma_{S}$ is of order of $\left(\operatorname{dist}_{\Gamma_{V}}\left(P, P_{0}\right)\right)^{k}$. 
Then the Levi form of $r$ in the direction $\zeta_{S}$ at a point $P$ in $\Gamma_{S}$ other than $P_{0}$ will change sign as $P$ moves along $\Gamma_{S}$ to pass $P_{0}$ because the evenness of $k$ implies that the Levi form of $r$ in the direction $\zeta_{S}$ at a point $P$ vanishes of odd order $k-1$ at $P_{0}$ along $\Gamma_{S}$. This contradicts the weak pseudoconvexity of $S$.

\section{Appendix A: Some Techniques of Applying of Skoda's Theorem on Ideal Generation}

In this Appendix we give some techniques of applying Skoda's theorem on ideal generation [Sk72, Th.1, pp.555-556] which involve derivatives and Jacobian determinants. The significance is more in the techniques themselves than in the statements given here to demonstrate their use. Though these techniques are not directly used in this note (except the use of (A.2) in (III.7) and the use of (A.3) in (III.8)), they may be useful in reducing the vanishing orders of multiplier ideals in Kohn-type algorithms in the setting of more general partial differential equations.

(A.1) Proposition. Let $\Omega$ be a bounded Stein open subset of $\mathbb{C}^{n}$. Let $g_{1}, \cdots, g_{n}, \rho$ be holomorphic functions on some open neighborhood $\tilde{\Omega}$ of the topological closure $\bar{\Omega}$ of $\Omega$. Let $Z$ be the common zero-set of $g_{1}, \cdots, g_{n}$ in $\tilde{\Omega}$. Assume that $\rho$ vanishes on $Z$. Let $J$ be the Jacobian determinant of $g_{1}, \cdots, g_{n}$. Then there exist holomorphic $h_{1}, \cdots, h_{n}$ on $\Omega$ such that $\rho J=\sum_{j=1}^{n} h_{j} g_{j}$.

Proof. For any $0<\gamma<1$ and any compact subset $K$ of $\mathbb{C}^{n}$ with coordinates $w=\left(w_{1}, \cdots, w_{n}\right)$ the integral

$$
\int_{w \in K} \frac{\prod_{j=1}^{n}\left(\sqrt{-1} d w_{j} \wedge d \overline{w_{j}}\right)}{\left(\sum_{j=1}^{n}\left|w_{j}\right|^{2}\right)^{\gamma n}}
$$

is finite. Since $\rho$ vanishes on $Z$, it follow that there exists some $0<\eta<1$ such that

$$
\frac{|\rho|^{2}}{\left(\sum_{j=1}^{n}\left|g_{j}\right|^{2}\right)^{\eta n}}
$$

is bounded on some open neighborhood $U$ of $\bar{\Omega}$ in $\tilde{\Omega}$. Let $\gamma=1-\frac{\eta}{2}$ and $\alpha=1+\frac{\eta}{2}$. Since $J$ is the Jacobian determinant of $g_{1}, \cdots, g_{n}$, by pulling back (A.1.1) by $w_{j}=g_{j}$ for $1 \leq j \leq n$ and using the uniform boundedness of (A.1.2) on $U$, we 
conclude that

$$
\int_{\Omega} \frac{|\rho J|^{2}}{\left(\sum_{j=1}^{n}\left|g_{j}\right|^{2}\right)^{\alpha n}}<\infty .
$$

By using (A.1.3) and applying Skoda's theorem [Sk72, Th.1, pp.555-556] to the Stein domain $\Omega$ to express $\rho J$ as a linear combination of $g_{1}, \cdots, g_{n}$ with holomorphic functions, we obtain $h_{1}, \cdots, h_{n}$ satisfying the requirements of the Proposition. Q.E.D.

(A.2) Proposition (Ideal Generated by Components of Gradient). Let $f$ be a holomorphic function germ on $\mathbb{C}^{n}$ at the origin which vanishes at the origin. Then $f^{n+1}$ belongs to the ideal $\mathcal{I}$ generated by $\frac{\partial f}{\partial z_{j}}$ for $1 \leq j \leq n$ at the origin, where $z_{1}, \cdots, z_{n}$ are the coordinates of $\mathbb{C}^{n}$.

Proof. Let $\pi: \tilde{U} \rightarrow U$ be the simultaneous resolution of singularities for the ideal $\mathcal{I}$ and the ideal $\mathcal{O}_{\mathbb{C}^{n}} f$ generated by $f$ with exceptional hypersurfaces $\left\{E_{\ell}\right\}_{\ell}$ in normal crossing in $\tilde{U}$, where $U$ is an open neighborhood of the origin in $\mathbb{C}^{n}$ on which the holomorphic function germ $f$ is defined. We claim that

$$
\frac{|f|^{2}}{\sum_{j=1}^{n}\left|\frac{\partial f}{\partial z_{j}}\right|^{2}}
$$

is uniformly bounded in some relatively compact open neighborhood $U^{\prime}$ of the origin in $U$. Otherwise, when we write the divisor of $\pi^{*} f$ of $f$ as $\sum_{\ell} a_{\ell} E_{\ell}$ and write $\pi^{*} \mathcal{I}$ as $\sum_{\ell} b_{\ell} E_{\ell}$ with $a_{\ell}$ and $b_{\ell}$ being nonnegative integers, we have $b_{\ell}>a_{\ell}$ for some $\ell$ with $0 \in \pi\left(E_{\ell}\right)$ and we can find a local holomorphic curve $\tilde{\varphi}: W \rightarrow \tilde{U}$ with $W$ being an open neighborhood of the origin in $\mathbb{C}$ and $\pi \tilde{\varphi}(0)=0$ such that $\varphi(W)$ is transversal to $E_{\ell}$ and is disjoint from any $E_{k}$ with $k \neq \ell$. Then $d(f \circ \varphi)$ vanishes at 0 to an order higher than that $f \circ \varphi$, which is a contradiction, because $f \circ \varphi$ vanishes at 0 . This argument actually gives a slightly higher vanishing order of $|f|^{2}$ than that of $\sum_{j=1}^{n}\left|\frac{\partial f}{\partial z_{j}}\right|^{2}$ along each $E_{\ell}$ when they are pulled back to $\tilde{U}$ so that

$$
\int_{U^{\prime}} \frac{\left|f^{n+1}\right|^{2}}{\left(\sum_{j=1}^{n}\left|\frac{\partial f}{\partial z_{j}}\right|^{2}\right)^{\alpha(n+1)}}<\infty
$$

for some $\alpha>1$. The conclusion of the Proposition now follows from Skoda's theorem [Sk72, Th.1, pp.555-556]. Q.E.D. 
(A.2.2) Remark on the Relation Between Proposition (A.2) and l'Hôpital's Rule. The argument in the proof of Proposition (A.2) consists of the verification of the uniform bound of (A.2.1) on some open neighborhood $U^{\prime}$ of the origin in $\mathbb{C}^{n}$ and a straightforward application of Skoda's theorem [Sk72, Th.1, pp.555556]. The argument used in the verification of the uniform bound of (A.2.1) on $U^{\prime}$ is actually the usual l'Hôpital's rule in calculus applied to the pullback of the quotient (A.2.1) to the open unit 1-disk $\Delta$ in $\mathbb{C}$ by a holomorphic map $g: \Delta \rightarrow \mathbb{C}^{n}$ with $g(0)=0$ when one applies differentiation at the origin along any ray of $\Delta$ until one ends up with a nonzero derivative of the denominator. The uniformity of the bound comes from the fact that one needs only consider a compact holomorphic family of such holomorphic maps $g: \Delta \rightarrow \mathbb{C}^{n}$, as is easily seen, for example, by using a resolution of singularities. Another simple way of looking at the bound of (A.2.1) is the trivial observation that the vanishing order of an analytic function at a point of its zero-set is no more than the vanishing order of its gradient.

(A.2.3) Remark on the Difference Between the Jacobian Determinants with Respect to All Variables and the Jacobian Determinants With Respect to All Variables with Respect to a Proper Subset of Variables. Let $F_{1}, \cdots, F_{N}$ be holomorphic function germs on $\mathbb{C}^{2}$ at the origin vanishing at the origin such that the ideal $I_{1}$ generated by $F_{1}, \cdots, F_{N}$ contains an effective power of the maximum ideal sheaf $\mathfrak{m}_{\mathbb{C}^{2}, 0}$ of $\mathbb{C}^{2}$. By Proposition (A.2) the ideal generated by the components of the gradients of $F_{1}, \cdots, F_{N}$, namely by $\frac{\partial F_{j}}{\partial z_{k}}$ for $1 \leq j \leq N, 1 \leq k \leq 2$, contains an effective power of $\mathfrak{m}_{\mathbb{C}^{2}, 0}$. We can regard each $\frac{\partial F_{j}}{\partial z_{k}}$ for $1 \leq j \leq N, 1 \leq k \leq 2$ as the Jacobian determinant of the single function $F_{j}$ with respect to the single variable $z_{j}$. These first-order partial derivatives can be regarded as the Jacobian determinants with respect to a proper subset of all the variables. Proposition (A.2) can be restated as follows. The ideal generated by all such Jacobian determinants with respect to a proper subset of all the variables contains an effective power of $\mathfrak{m}_{\mathbb{C}^{2}, 0}$. The situation is very different from the ideal $I_{2}$ generated by all Jacobian determinants with respect to the full set of all the variables

$$
\frac{\partial\left(F_{j_{1}}, F_{j_{2}}\right)}{\partial\left(z_{1}, z_{2}\right)} \text { for } 1 \leq j_{1}, j_{2} \leq N
$$

In general, the ideal $I_{2}$ does not contain an effective power of $\mathfrak{m}_{\mathbb{C}^{2}, 0}$, as one can easily see in the special case where $N=2$ and the ideal $I_{2}$ is generated by a single holomorphic function germ. 
In general, for the complex Euclidean space $\mathbb{C}^{n}$ instead of $\mathbb{C}^{2}$, when we have holomorphic function germs $F_{1}, \cdots, F_{N}$ on $\mathbb{C}^{n}$ at the origin vanishing at the origin such that the ideal generated by $F_{1}, \cdots, F_{N}$ contains an effective power of the maximum ideal sheaf $\mathfrak{m}_{\mathbb{C}^{n}, 0}$ of $\mathbb{C}^{n}$, we can consider for $1 \leq \nu \leq n$ the ideal $I_{\nu}$ generated by the Jacobian determinants

$$
\frac{\partial\left(F_{j_{1}}, \cdots, F_{j_{\nu}}\right)}{\partial\left(z_{k_{1}}, \cdots, z_{k_{\nu}}\right)}
$$

for $1 \leq j_{1}, \cdots, j_{\nu} \leq N$ and $1 \leq k_{1}, \cdots, k_{\nu} \leq n-1$. As we see in Proposition (A.3) below, for $1 \leq \nu \leq n-1$ the ideal $I_{\nu}$ contains an effective power of $\mathfrak{m}_{\mathbb{C}^{n}, 0}$, though in general the ideal $I_{n}$ does not contain an effective power of $\mathfrak{m}_{\mathbb{C}^{n}, 0}$. It means that the situation for the ideal generated by all Jacobian determinants with respect to a proper subset of all the variables is very different from the ideal $I_{2}$ generated by all Jacobian determinants with respect to the full set of all the variables.

(A.2.4) Remark on a Generalization of the Special Case of Proposition (A.2) for Dimension Two. The special case of Proposition (A.2) for dimension two is used in this note in (III.7) to prove the effective termination of Kohn's algorithm for $\mathbb{C}^{2}$. For the proof of the effective termination of Kohn's algorithm for $\mathbb{C}^{n}$ the corresponding statement which has to be used is not Proposition (A.2) for dimension $n$, but the following Proposition (A.3).

(A.3) Proposition (Ideal Generated by Jacobian Determinants with Respect to a Proper Subset of Variables). Let $F_{1}, \cdots, F_{N}$ be holomorphic function germs on $\mathbb{C}^{n}$ at the origin vanishing at the origin such that the ideal generated by $F_{1}, \cdots, F_{N}$ contains an effective power of the maximum ideal of $\mathbb{C}^{n}$ at the origin. Let $1 \leq \nu<n$. Let $J_{\nu}$ be the ideal generated by

$$
\frac{\partial\left(F_{j_{1}}, \cdots, F_{j_{\nu}}\right)}{\partial\left(z_{k_{1}}, \cdots, z_{k_{\nu}}\right)} \text {. }
$$

for $1 \leq j_{1}<\cdots<j_{\nu} \leq N$ and $1 \leq k_{1}<\cdots<k_{\nu} \leq n$. Then the ideal $J_{\nu}$ contains an effective power of the maximum ideal of $\mathbb{C}^{n}$ at the origin.

Proof. Let us first introduce some notations. For an ideal $I$ of $\mathcal{O}_{\mathbb{C}^{n}, 0}$ we define

$$
\left|s_{I}\right|=\left(\sum_{j=1}^{k_{I}}\left|s_{j, I}\right|^{2}\right)^{\frac{1}{2}}
$$


where $s_{1, I}, \cdots, s_{k_{I}, I}$ form a set of generators of $I$. The expression $\left|s_{I}\right|$ is defined up to a choice of the set of generators. We use this expression only in the context of determining whether one such expression is dominated by a constant times another such expression $\left|s_{J}\right|$ for another ideal $J$ of $\mathcal{O}_{\mathbb{C}^{n}, 0}$. For such a purpose the choices of generators in the definitions for $\left|s_{I}\right|$ and $\left|s_{J}\right|$ are immaterial. For our purpose, if $\lambda \in \mathbb{N}$ and $\hat{I}$ is $I^{\lambda}$, then we can use $\left|s_{\hat{I}}\right|=\left|s_{I}\right|^{\lambda}$. For a holomorphic map $\psi: \Delta \rightarrow \mathbb{C}^{n}$ with $\psi(0)=0$ and an ideal $I$ of $\mathcal{O}_{\mathbb{C}^{n}, 0}$ with generators $s_{1, I}, \cdots, s_{k_{I}, I}$, by the vanishing order $a_{I, \psi}$ of $I$ on $\psi$ at 0 we mean the minimum of $\operatorname{ord}_{0}\left(s_{j, I} \circ \psi\right)$ for $1 \leq j \leq k_{I}$, where $\operatorname{ord}_{0}(\cdot)$ denotes the vanishing order on $\mathbb{C}$ at the origin. For an $\ell$-jet $\xi$ of $\mathbb{C}^{n}$ at the origin which can be represented by $\psi$ we denote $a_{I, \psi}$ also by $a_{I, \xi}$. (Here the convention is that a 1-jet is a tangent vector.) If $a_{I, \psi}<\ell$, then $a_{I, \xi}=a_{I, \varphi}$ for any holomorphic map $\varphi: \Delta \rightarrow \mathbb{C}^{n}$ with $\varphi(0)=0$ which represents the $\ell$-jet $\xi$.

Note that for our purpose we could also use alternatively the concept of the normalized vanishing order of $I$ on $\psi$ at 0 (instead of the vanishing order $a_{I, \psi}$ ) by defining the normalized vanishing order of $I$ on $\psi$ at 0 as the minimum of

$$
\frac{\operatorname{ord}_{0}\left(s_{j, I} \circ \psi\right)}{\operatorname{ord}_{0} \psi}
$$

for $1 \leq j \leq k_{I}$, where $\operatorname{ord}_{0} \psi$ is the minimum of the vanishing orders of the $n$ components of $\psi$ on $\mathbb{C}$ at the origin.

Since all the main arguments in this proof occur already in the proof of the special case where $N=n=3$, for notational simplicity we will only present the proof of this special case. The general case is completely analogous but with much more complicated notations. We break down the proof into the following five steps.

Step One. Let $G_{1}, G_{2}$ be holomorphic function germs on $\mathbb{C}^{3}$ at the origin vanishing at the origin such that the divisor $Z_{1}$ of $G_{1}$ is irreducible and of multiplicity 1. Assume that $d G_{1} \wedge d G_{2}$ is not identically zero. Then there exists some positive constant $C$ such that

$$
\sum_{k_{1}, k_{2}=1}^{3}\left|G_{2}\left(d G_{1} \wedge d z_{k_{1}} \wedge d z_{k_{2}}\right)\right|^{2} \leq C \sum_{j=1}^{3}\left|d G_{1} \wedge d G_{2} \wedge d z_{j}\right|^{2}
$$

on $Z_{1}=\left\{G_{1}=0\right\}$.

Step One is verified by 
(i) taking any holomorphic curve $\varphi: \Delta \rightarrow G_{1}$ with $\varphi(0)=0$ and the image of $\varphi(\Delta)$ not contained in the zero-set of $G_{2}$,

(ii) using the fact that the vanishing order at the origin of the pullback $G_{2} \circ \varphi$ on $\Delta$ is no more than the minimum of the vanishing orders of its first-order partial derivatives at the origin, and

(iii) observing that at a regular point of $Z_{1}$, where $z_{k_{1}}, z_{k_{2}}$ are used as local coordinates, the component of the gradient of the restriction of $G_{2}$ to $Z_{1}$ for the coordinate $z_{k_{1}}$ is equal to the quotient of $d G_{1} \wedge d G_{2} \wedge d z_{k_{2}}$ by $d G_{1} \wedge d z_{k_{1}} \wedge d z_{k_{2}}$ as one can easily see by using the chain rule and the implicit differentiation for functions defined on $Z_{1}=\left\{G_{1}=0\right\}$.

Step Two. Let $I$ and $J$ be ideals in $\mathcal{O}_{\mathbb{C}^{3}, 0}$ contained in the maximum ideal $\mathfrak{m}_{\mathbb{C}^{3}, 0}$ of $\mathcal{O}_{\mathbb{C}^{3}, 0}$ such that $I$ contains $\left(\mathfrak{m}_{\mathbb{C}^{n}, 0}\right)^{q}$ for some positive integer $q$. If $\left|s_{I}\right|$ is not dominated by a constant times $\left|s_{J}\right|$, then there exists some $(q+2)$-jet $\xi$ of $\mathbb{C}^{3}$ at the origin which is represented by some holomorphic map $\psi: \Delta \rightarrow \mathbb{C}^{3}$ with $\psi(0)=0$ such that $a_{I, \psi} \leq q$ and $a_{I, \psi}<a_{J, \psi}$.

Step Three. Let $A$ be the ideal generated by elements $F_{1}, F_{2}, F_{3}$ of $\mathfrak{m}_{\mathbb{C}^{3}, 0}$ such that $A$ contains $\left(\mathfrak{m}_{\mathbb{C}^{3}, 0}\right)^{q}$ for some positive integer $q$. Let $p \in \mathbb{N}$. Then there exists a positive integer $q_{1}$ depending only on $q$ and there exists a positive integer $m$ depending on $q$ and $p$ with the following property. For any $p$-jet $\xi$ of $\mathbb{C}^{3}$ at the origin, let $P_{1}\left(F_{1}, F_{2}, F_{3}\right)$ be a generic homogeneous polynomial of degree $m$ in $F_{1}, F_{2}, F_{3}$ whose divisor $V$ contains a holomorphic curve representing $\xi$ and let $\varphi: \Delta \rightarrow \mathbb{C}^{3}$ be a holomorphic curve germ with $\varphi(0)=0$ whose image is a generic curve germ in $V$ which represents $\xi$. Then the minimum vanishing order of $\frac{\partial}{\partial z_{\ell}} P_{1}\left(F_{1}, F_{2}, F_{3}\right)$ on the holomorphic curve germ $\varphi: \Delta \rightarrow \mathbb{C}^{3}$ at the origin is no more than $(m-1) a_{A, \varphi}+q_{1}$ for $1 \leq \ell \leq 3$.

Note that $V$, as the divisor of a generic homogeneous polynomial of degree $m$ in $F_{1}, F_{2}, F_{3}$ which contains a holomorphic curve representing $\xi$, is a reduced and irreducible hypersurface germ in $\mathbb{C}^{3}$ at the origin. Moreover, the image of $\varphi: \Delta \rightarrow \mathbb{C}^{3}$, as a generic curve germ in $V$ which represents $\xi$, is contained in $\{0\} \cup \operatorname{Reg}(V)$, where $\operatorname{Reg}(V)$ is the regular part of $V$.

Step Four. Let $F_{1}, F_{2}, F_{3}$ be from Step Three. Let $J$ be the ideal generated by

$$
\frac{\partial\left(F_{j_{1}}, F_{j_{2}}\right)}{\partial\left(z_{k_{1}}, z_{k_{2}}\right)}
$$


for $1 \leq j_{1}<j_{2} \leq 3$ and $1 \leq k_{1}<k_{2} \leq 3$. Let $\lambda \in \mathbb{N}$ and $I=\left(\mathfrak{m}_{\mathbb{C}^{3}, 0}\right)^{\lambda}$. Let $p=\lambda+2$. Assume that $\left|s_{I}\right|$ is not dominated by any positive constant times $\left|s_{J}\right|$. By Step Two, there exists a $p$-jet $\xi$ of $\mathbb{C}^{3}$ at origin such that, for any holomorphic map $\varphi: \Delta \rightarrow \mathbb{C}^{3}$ whose $p$-jet at the origin is equal to $\xi$, the vanishing order $a_{J, \varphi}$ of $J$ on $\varphi$ at 0 is greater than the vanishing order $a_{I, \varphi}$ of $I$ on $\varphi$ at the origin. By Step Three we have positive integers $q_{1}, m$ (with $q_{1}$ depending only on $p$ and with $m$ depending only on $p$ and $q)$ and we have a polynomial $P_{1}\left(F_{1}, F_{2}, F_{3}\right)$ homogeneous of degree $m$ in $F_{1}, F_{2}, F_{3}$ and a holomorphic curve germ $\varphi: \Delta \rightarrow \mathbb{C}^{3}$ at the origin such that

(i) the divisor of $P_{1}\left(F_{1}, F_{2}, F_{3}\right)$ is a reduced and irreducible hypersurface germ of $\mathbb{C}^{3}$ at the origin,

(ii) the image of the holomorphic curve germ $\varphi: \Delta \rightarrow \mathbb{C}^{3}$ is contained in the divisor of $P_{1}\left(F_{1}, F_{2}, F_{3}\right)$,

(iii) the holomorphic curve germ $\varphi: \Delta \rightarrow \mathbb{C}^{3}$ represents the $p$-jet $\xi$ of $\mathbb{C}^{3}$ at the origin,

(vi) the minimum vanishing order of $\frac{\partial}{\partial z_{\ell}} P_{1}\left(F_{1}, F_{2}, F_{3}\right)$ on the holomorphic curve germ $\varphi: \Delta \rightarrow \mathbb{C}^{3}$ is no more than $(m-1) a_{A, \varphi}+q_{1}$ for $1 \leq \ell \leq 3$.

For any polynomial $P_{2}\left(F_{1}, F_{2}, F_{3}\right)$ of degree $m$ in $F_{1}, F_{2}$ and for $1 \leq k_{1}<k_{2} \leq 3$, it follows from

$$
\frac{\partial P_{j}}{\partial z_{k}}=\sum_{\ell=1}^{3} \frac{\partial P_{j}}{\partial F_{\ell}} \frac{\partial F_{\ell}}{\partial z_{k}}
$$

by the chain rule that

$$
\frac{\partial\left(P_{1}, P_{2}\right)}{\partial\left(z_{k_{1}}, z_{k_{2}}\right)}=\sum_{\ell_{1}, \ell_{2}=1}^{3} \frac{\partial P_{1}}{\partial F_{\ell_{1}}} \frac{\partial P_{2}}{\partial F_{\ell_{2}}} \frac{\partial\left(F_{\ell_{1}}, F_{\ell_{2}}\right)}{\partial\left(z_{k_{1}}, z_{k_{2}}\right)} .
$$

The vanishing order of

$$
\frac{\partial\left(P_{1}, P_{2}\right)}{\partial\left(z_{k_{1}}, z_{k_{2}}\right)}
$$

on the holomorphic curve germ $\varphi: \Delta \rightarrow \mathbb{C}^{3}$ is at least $2(m-1) a_{A, \varphi}+a_{J, \varphi}$.

Applying Step One to the case of $G_{1}=P_{1}\left(F_{1}, F_{2}, F_{3}\right)$ and $G_{2}=P_{2}\left(F_{1}, F_{2}, F_{3}\right)$ with $P_{2}\left(F_{1}, F_{2}, F_{3}\right)$ being any generic polynomial homogeneous of degree $m$ in $F_{1}, F_{2}, F_{3}$, we get

$$
\sum_{k_{1}, k_{2}=1}^{3}\left|P_{2}\left(d P_{1} \wedge d z_{k_{1}} \wedge d z_{k_{2}}\right)\right|^{2} \leq C \sum_{j=1}^{3}\left|d P_{1} \wedge d P_{2} \wedge d z_{j}\right|^{2}
$$


on $P_{1}=0$, where $C$ is a positive constant. We restrict this inequality to the curve $\varphi$ and conclude that

$$
m a_{A, \varphi}+(m-1) a_{A, \varphi}+q_{1} \geq 2(m-1) a_{A, \varphi}+a_{J, \varphi} .
$$

By the choice of $P_{1}\left(F_{1}, F_{2}, F_{3}\right)$ and the holomorphic curve germ $\varphi: \Delta \rightarrow \mathbb{C}^{3}$, we have $a_{J, \varphi}>\lambda$. Thus

$$
m a_{A, \varphi}+(m-1) a_{A, \varphi}+q_{1} \geq 2(m-1) a_{A, \varphi}+\lambda
$$

and we conclude that $\lambda \leq a_{A, \varphi}+q_{1} \leq q+q_{1}$, because $a_{A, \varphi} \leq q$ from the fact that $A$ contains $\left(\mathfrak{m}_{\mathbb{C}^{3}, 0}\right)^{q}$.

Step Five. By setting $\lambda=q+q_{1}+1$, we conclude from Step Four that $\left|s_{I}\right|$ is dominated by a constant times $\left|s_{J}\right|$. As in the last part of the proof of Proposition (A.2), by Skoda's theorem [Sk72, Th.1, pp.555-556] it follows from the local integrability of the quotient

$$
\frac{\left|s_{I}\right|^{2(n+2)}}{\left|s_{J}\right|^{2(n+2)}}
$$

on $\mathbb{C}^{3}$ at the origin that $\left(\mathfrak{m}_{\mathbb{C}^{3}, 0}\right)^{\left(q+q_{1}+1\right)(n+2)}$ is contained in the ideal $J$ generated by

$$
\frac{\partial\left(F_{j_{1}}, F_{j_{2}}\right)}{\partial\left(z_{k_{1}}, z_{k_{2}}\right)}
$$

for $1 \leq j_{1}<j_{2} \leq 3$ and $1 \leq k_{1}<k_{2} \leq 3$. This finishes the proof.

We would like to remark that the main point of this proof is to apply the argument for gradients given in Proposition (A.2) for $\mathbb{C}^{n}$ to the divisor of $P_{1}\left(F_{1}, F_{2}, F_{3}\right)$ in $\mathbb{C}^{3}$ instead of to $\mathbb{C}^{n}$. Q.E.D.

(A.4) Proposition. Let $h_{1}, \cdots, h_{n}$ be holomorphic function germs on $\mathbb{C}^{n}$ at the origin so that the origin is their only common zero. Let $d h_{1} \wedge \cdots \wedge d h_{n}=$ $J\left(d z_{1} \wedge \cdots \wedge d z_{n}\right)$. Then $J$ does not belong to the ideal generated by $h_{1}, \cdots, h_{n}$.

Proof. Suppose the contrary. Then there exist holomorphic function germs $f_{1}, \cdots, f_{n}$ on $\mathbb{C}^{n}$ at the origin such that $J=\sum_{j=1}^{n} f_{j} h_{j}$. We let $\omega_{j}=f_{j}\left(d z_{1} \wedge \cdots \wedge d z_{n}\right)$ for $1 \leq j \leq n$ so that

$$
d h_{1} \wedge \cdots \wedge d h_{n}=\sum_{j=1}^{n} h_{j} \omega_{j} .
$$


Since the origin is the only common zero of $h_{1}, \cdots, h_{n}$, we can find connected open neighborhoods $U$ and $W$ of the origin in $\mathbb{C}^{n}$ so that the map $\pi: \mathbb{C}^{n} \rightarrow \mathbb{C}^{n}$ defined by

$$
\left(z_{1}, \cdots, z_{n}\right) \mapsto\left(w_{1}, \cdots, w_{n}\right)=\left(h_{1}\left(z_{1}, \cdots, z_{n}\right), \cdots, h_{n}\left(z_{1}, \cdots, z_{n}\right)\right)
$$

maps $U$ properly and surjectively onto $W$ and makes $U$ a branched cover over $W$ of $\lambda$ sheets. By replacing $U$ and $W$ by relatively compact open neighborhoods $U^{\prime}$ and $W^{\prime}$ of the origin in $U$ and $W$ respectively, we can assume without loss of generality that $\int_{U}\left|\omega_{j}\right|^{2} \leq C<\infty$ for $1 \leq j \leq n$. We take the direct image of the equation (A.4.1) under $\pi$. The left-hand side of the equation (A.4.1) yields $\lambda\left(d w_{1} \wedge \cdots \wedge d w_{n}\right)$, because the map $\pi$ is defined by $w_{j}=h_{j}$ for $1 \leq j \leq n$. Let $\theta_{j}$ be the direct image of $\omega_{j}$ under $\pi$ for $1 \leq j \leq n$. Let $Z$ be the branching locus of $\pi$ in $W$. For any simply connected open subset $G$ of $W-Z, U \cap \pi^{-1}(G)$ is the disjoint union of $\lambda$ open subsets $H_{1}, \cdots, H_{\lambda}$ of $U$ and $\theta_{j}(Q)=\sum_{\ell=1}^{\lambda} \omega_{j}\left(\tilde{Q}_{j}\right)$, where $U \cap \pi^{-1}(Q)=\left\{\tilde{Q}_{1}, \cdots, \tilde{Q}_{\lambda}\right\}$ with $\tilde{Q}_{j} \in H_{j}$. Now

$$
\int_{G}\left|\theta_{j}\right|^{2} \leq \lambda \sum_{j=1}^{\lambda} \int_{H_{j}}\left|\omega_{j}\right|^{2} \leq \lambda C .
$$

Since $W-Z$ can be covered by a finite number of simply connected open subsets, it follows that

$$
\int_{G}\left|\theta_{j}\right|^{2}<\infty \quad \text { for } 1 \leq j \leq n
$$

Thus $\theta_{j}$ is a holomorphic $n$-form on $G$ and

$$
\lambda d z_{1} \wedge \cdots \wedge d z_{n}=\sum_{j=1}^{n} z_{j} \theta_{j}
$$

on $G$, which gives a contradiction, because the left-hand side does not vanish at the origin whereas the right-hand side does. Q.E.D.

(A.5) Remark. Proposition (A.4) uses only the direct images of top-degree holomorphic forms and actually does not use Skoda's theorem [Sk72, Th.1, pp.555556]. The significance of Proposition (A.4) is that the coefficient $J$ in

$$
d h_{1} \wedge \cdots \wedge d h_{n}=J\left(d z_{1} \wedge \cdots \wedge d z_{n}\right)
$$


cannot be contained in $\left(\mathfrak{m}_{\mathbb{C}^{n}, 0}\right)^{p}$ if

$$
\left(\mathfrak{m}_{\mathbb{C}^{n}, 0}\right)^{p} \subset \sum_{j=1}^{n} \mathcal{O}_{\mathbb{C}^{n}, 0} h_{j}
$$

so that the vanishing order of $J$ at 0 is no more than $p$.

(A.5) Example to Show the Sharpness of the Exponent in Skoda's Theorem. The exponent in the denominator of the assumption in Skoda's theorem [Sk72, Th.1, pp.555-556] plays a rôle in effective bounds. As stated in Skoda's theorem [Sk72, Th.1, pp.555-556] it is sharp and cannot be lowered even in the case of Riemann surfaces. Let $X$ be the Riemann sphere $\mathbb{P}_{1}$. Consider the hyperplane section line bundle $H_{\mathbb{P}_{1}}$. Take two holomorphic sections $g_{1}, g_{2}$ of $H_{\mathbb{P}_{1}}$ without common zeroes. Take the holomorphic section $f$ of $2 H_{\mathbb{P}_{1}}+K_{\mathbb{P}_{1}}$ over $\mathbb{P}_{1}$ which corresponds to a constant function on $\mathbb{P}_{1}$ via the isomorphism between $K_{\mathbb{P}_{1}}$ and $-2 H_{\mathbb{P}_{1}}$. If the exponent used in the denominator of the assumption in Skoda's theorem [Sk72, Th.1, pp.555-556] can be lowered so that $\alpha=1$, then $p=2$ and $n=1$ and $q=\min (n, p-q)=1$ and $\alpha q+1=2$ and the assumption

$$
\int_{\mathbb{P}_{1}} \frac{|f|^{2}}{\left(\left|g_{1}\right|^{2}+\left|g_{2}\right|^{2}\right)^{\alpha q+1}}<\infty
$$

is satisfied because $g_{1}, g_{2}$ have no common zeroes. Note that when $\alpha>1$, the integrand of the above inequality makes no sense unless

$$
f \in \Gamma\left(\mathbb{P}_{1}, m H_{\mathbb{P}_{1}}+K_{\mathbb{P}_{1}}\right)
$$

for some $m>2$. If Skoda's theorem [Sk72, Th.1, pp.555-556] holds with the lower exponent in the denominator in its assumption, then we can write $f=h_{1} g_{1}+h_{2} g_{2}$ with

$$
h_{1}, h_{2} \in \Gamma\left(\mathbb{P}_{1}, H_{\mathbb{P}_{1}}+K_{\mathbb{P}_{1}}\right)
$$

which is impossible, because

$$
\Gamma\left(\mathbb{P}_{1}, H_{\mathbb{P}_{1}}+K_{\mathbb{P}_{1}}\right)=0
$$

from the isomorphism between $K_{\mathbb{P}_{1}}$ and $-2 H_{\mathbb{P}_{1}}$.

\section{References}

[Ca84] D. Catlin, Boundary invariants of pseudoconvex domains. Ann. of Math. 120 (1984), 529-586. 
[DA79] J. P. D'Angelo, Finite type conditions for real hypersurfaces. J. Diff. Geom. 14 (1979), 59-66.

[DF77] K. Diederich and J. E. Fornaess, Pseudoconvex domains: bounded strictly plurisubharmonic exhaustion functions. Invent. Math. 39 (1977), 129-141.

[DF78] K. Diederich and J. E. Fornaess, Pseudoconvex domains with real-analytic boundary. Ann. of Math. 107 (1978), 371-384.

[GT83] D. Gilbarg and N. Trudinger, Elliptic partial differential equations of second order. Second edition. Grundlehren der Mathematischen Wissenschaften, Volume 224. Springer-Verlag, Berlin, 1983.

[He62] S. Helgason, Differential Geometry and Symmetric Spaces, Academic Press 1962.

[Ko79] J. J. Kohn, Subellipticity of the $\bar{\partial}$-Neumann problem on pseudo-convex domains: sufficient conditions. Acta Math. 142 (1979), 79-122.

[Ni07] Andreea C. Nicoara, Equivalence of types and Catlin boundary systems, arXiv:0711.0429 (November 2007).

[Sk72] H. Skoda, Application des techniques $L^{2}$ à la théorie des idéaux d'une algèbre de fonctions holomorphes avec poids, Ann. Sci. Ec. Norm. Sup. $\mathbf{5}$ (1972), 548-580.

[To72] J.-C. Tougeron, Idéaux de fonctions différentiables. Ergebnisse der Mathematik und ihrer Grenzgebiete, Volume 71. Springer-Verlag, Berlin-New York, 1972.

[ZS60] O. Zariski and P. Samuel, Commutative algebra. Vol. II. The University Series in Higher Mathematics. D. Van Nostrand Co., Inc., Princeton, N. J.Toronto-London-New York 1960.

Yum-Tong Siu

Department of Mathematics

Harvard University,

Cambridge, MA 02138, U.S.A

E-mail: siu@math.harvard.edu 